NBER WORKING PAPER SERIES

\title{
INVESTMENT UNDER UNCERTAINTY WITH FINANCIAL CONSTRAINTS
}

\author{
Patrick Bolton \\ Neng Wang \\ Jinqiang Yang \\ Working Paper 20610 \\ http://www.nber.org/papers/w20610 \\ NATIONAL BUREAU OF ECONOMIC RESEARCH \\ 1050 Massachusetts Avenue \\ Cambridge, MA 02138 \\ October 2014, Revised July 2019
}

We are deeply grateful to Pierre-Olivier Weill for his insightful and detailed comments. This paper was previously circulated under the title "Investment under Uncertainty and the Value of Real and Financial Flexibility" (2014, NBER wp 20601.) We thank Andrew Abel, Ilona Babenka, Martin Cherkes, Min Dai, Sudipto Dasgupta, Peter DeMarzo, Janice Eberly, Mark Gertler, Simon Gilchrist, Steve Grenadier, Lars Peter Hansen, Vicky Henderson, Robert Hodrick, Harrison Hong, Wei Jiang, Bart Lambrecht, Erica Xuenan Li, Xiaoji Lin, Robert McDonald, Stewart Myers, Alessandro Pavan (Editor), Robert Pindyck, Tom Sargent, Suresh Sundaresan, Vladimir Vladimirov, and seminar participants at American Finance Association (AFA) 2014 meetings in Philadelphia, Boston University, China International Conference in Finance (CICF), Columbia University, HKUST 2013 Finance Symposium, the Paul Woolley Centre Conference at UTS Sydney, Rutgers University, University of Cambridge, University of Minnesota Carlson School, Zhejiang University, and the workshops hosted by the China Young Finance Scholars Society for helpful comments. Jinqiang Yang acknowledges support from the National Natural Science Foundation of China (71772112, 71472117, 71573033, 71532009, and 71532012) and Innovative Research Team of Shanghai University of Finance and Economics (\#2016110241) The views expressed herein are those of the authors and do not necessarily reflect the views of the National Bureau of Economic Research.

NBER working papers are circulated for discussion and comment purposes. They have not been peer-reviewed or been subject to the review by the NBER Board of Directors that accompanies official NBER publications.

(C) 2014 by Patrick Bolton, Neng Wang, and Jinqiang Yang. All rights reserved. Short sections of text, not to exceed two paragraphs, may be quoted without explicit permission provided that full credit, including $\odot$ notice, is given to the source. 
Investment under Uncertainty with Financial Constraints

Patrick Bolton, Neng Wang, and Jinqiang Yang

NBER Working Paper No. 20610

October 2014, Revised July 2019

JEL No. E22,G31,G32

\begin{abstract}
$\underline{\text { ABSTRACT }}$
We develop an integrated theory of investment, seasoned equity offerings (SEOs), liquidation, and corporate savings under uncertainty for a financially constrained firm, which features endogenous growth options, abandonment options, and payout policies. Facing costly external financing, the firm prefers to fund its investment internally, so that its optimal policies and value depend on both its earnings fundamentals and liquidity holdings. The firm values not only real flexibility but also financial flexibility. The interaction of real and financial flexibility generates novel real options results: (1) Limited financial slack significantly erodes the value of growth \& abandonment options; (2) Firms prefer projects with front-loaded cash-flows; (3) The firm's incentive to forgo costly external financing and to accumulate internal funds may cause substantial delay in investment; (4) A financially constrained firm over-invests in early stages of its life-cycle in an effort to quickly build up its cash-flow generating capacity; (5) SEOs are driven by both firm survival and growth motives. A firm in the mature phase may find itself in three mutually exclusive regions: payout, inaction, and liquidation. A firm in its growth phase may find itself in two additional regions: a region where investment is partly financed with an SEO and a region where investment is solely financed with internal funds. These regions depend on both firm savings and earnings fundamentals.

Patrick Bolton

Columbia Business School

804 Uris Hall

New York, NY 10027

and NBER

pb2208@columbia.edu

Neng Wang

Columbia Business School

3022 Broadway, Uris Hall 812

New York, NY 10027

and NBER

nw2128@columbia.edu

Jinqiang Yang

Shanghai University of Finance

and Economics

Guoding Rd. 777

Shanghai, 200433

China

yang.jinqiang@mail.sufe.edu.cn
\end{abstract}




\section{Introduction}

Real-options theory following McDonald and Siegel (1986) and Dixit and Pindyck (1994) assumes that firms operate in a Modigliani-Miller (MM) environment. This is for a good reason. The firm's investment decision can then be formulated as an American option-exercise and valuation problem that can be solved by using the classical option pricing tools of Black and Scholes (1973) and Merton (1973).

But, in reality firms operate under imperfect capital markets and face significant external financing costs that arise from informational asymmetry, moral hazard, transaction costs, and other frictions. In order to avoid incurring these costs too frequently firms optimally retain earnings and attempt to mostly internally finance their investments and cover their operating costs. Indeed, according to Chen, Karabarbounis, and Neiman (2017), "nowadays nearly two-thirds of global investment is funded by corporate saving." Also the surveys of chief financial officers (CFOs) by Graham and Harvey $(2001,2002)$ have revealed the great importance CFOs attach to maintaining financial flexibility by holding internal funds.

In practice, the value of real flexibility derived from the optimal timing of real options is intertwined with the value of financial flexibility derived from the optimal management of retained earnings and the optimal external financing timing and issuance decisions. Both the effective discount rate used to determine the value of a growth opportunity and the cost of investment depend on the firm's marginal value of cash, which varies with both the size of the firm's cash holdings and the firm's earnings fundamentals. The reason is that the firm takes account not only of its current stock of internal funds but also the information about its future cash flow prospects contained in persistent earnings shocks. Through the firm's evolving marginal value of cash, the real option problem becomes a fundamentally two-dimensional problem, which entails a significant generalization of the classical one-dimensional problems of McDonald and Siegel (1986) and Dixit and Pindyck (1994).

We embark on such a generalization in this paper and derive how optimal investmenttiming and abandonment-timing decisions are made, as well as how assets in place and growth options are valued, when firms face external financing costs. By introducing external financing costs, we are, in effect, integrating two strands of literature, the classical real options literature following McDonald and Siegel $(1985,1986)$ and Dixit and Pindyck (1994) with the corporate finance literature following Miller and 
Orr (1966). Our continuous-time model allows us to not only derive optimal investment, abandonment, and equity issue size and timing policies but also to carry out a systematic valuation analysis.

We model the firm's life-cycle and its evolving collection of assets in place and growth opportunities in the spirit of Myers (1977). Although we do not explicitly model adverse selection, one interpretation of costly external financing in our model is equity dilution cost (due to mis-pricing a la arguments in Myers and Majluf (1984).

In our model, the firm's investment, financing, payout and abandonment policies depend on both earnings fundamentals and the firm's financial slack. Therefore, our model can explain why following a recession low investment persists even though earnings fundamentals have recovered. The reason is that following a recession firms are generally in repair mode, seeking to rebuild their financial slack. Similarly, in our model firms have a preference for investments with front-loaded earnings. We show that, as a result, a start-up firm may choose to invest in a project sooner than predicted by the classical real-option theory in an effort to build future internal funding capacity. We also show that the firm's payout policy is fundamentally different depending on whether it is in the growth phase or in the mature phase. In the mature phase a more profitable firm pays out more, while in the growth phase it pays out less.

Our model also predicts that the value of growth and abandonment options effectively vanishes for firms with low internal financing ability. Firms with scarce internal funds are forced into inefficiently liquidating their valuable operating assets. We also find that the hurdle for investment in the growth phase is a non-monotonic function of the firm's internal funds. When the firm's savings are sufficient to entirely cover the investment cost, the firm's investment hurdle decreases with its internal funds. But when its internal funds cannot entirely cover the cost of investment, the hurdle is increasing with the firm's internal funds. The reason is that when the firm is approaching the point where it may be able to entirely fund its investment with retained earnings it has a stronger and stronger incentive to delay investment until it has sufficient funds to be able to avoid tapping costly external funds. An important implication of this result is that investment is not necessarily more likely when the firm has more cash. Investment could well be delayed further, as the firm's priority becomes avoiding costly external financing.

We also show that financially constrained firms prefer growth opportunities with 
front-loaded cash-flows. In the MM-based real options framework there is an equivalence between an option that pays cash flows over time and an option that pays immediately upon exercising a lump sum with the same present value. This is not true in our model for a financially constrained firm. Not only is the value of real options with more front-loaded cash-flows higher but also the firm may be induced to over-invest. More generally, a financially constrained firm cares not only about the present value of an investment project but also the project's payback period.

Another subtle prediction is that the amount of external equity financing is also non-monotonic in the profitability of the firm's operating assets. The intuition is as follows. For a firm whose investment option is sufficiently out of the money, financial flexibility has little value. On the other hand, a firm that is so profitable that its investment option is deep in the money can easily generate cash from operations. Therefore, funds obtained through an equity issue are most valuable for a firm whose profitability is in the medium range. In sum, for firms facing external financing costs the value of real options is not just tied to the timing of the real option but also to financial flexibility. Hugonnier, Malamud, and Morellec (2015) consider a model of costly and uncertain external financing in which the amount of external financing may also be non-monotonic in profitability. Décamps, Gryglewicz, Morellec, and Villeneuve (2017) obtain a similar prediction in a model with both permanent and transitory shocks.

A real options theory that includes external financing costs is obviously on a substantially stronger empirical footing than the classical theory that assumes perfect capital markets. This is evident with respect to the empirical evidence in Kim and Weisbach (2008) on global seasoned equity offerings (SEOs) and DeAngelo, DeAngelo and Stulz (2010) on SEOs of U.S. industrial corporations. The former paper finds that a large fraction of the SEO proceeds is saved inside the firm and savings are larger for growth firms. The latter finds that SEOs are first and foremost lastresort financing decisions for firms running out of cash, or in need of funds towards anticipated investment outlays. Although they find evidence in support of the market timing hypothesis of Loughran and Ritter $(1995,1997)$ and Baker and Wurgler (2002) on the one hand, and the life-cycle hypothesis on the other, ${ }^{1}$ these hypothe-

\footnotetext{
${ }^{1}$ By the life-cycle hypothesis, they mean that equity issuers are primarily young firms with high market-to-book (M/B) ratios and low operating cash flows, and mature firms with low M/B ratios pay dividends and fund investments out of internal funds.
} 
ses do not explain most of the SEOs that are observed. The main conclusion that DeAngelo, DeAngelo and Stulz (2010) draw is that "paraphrasing Sherlock Holmes, 'many dogs don't bark' at times when according to theory they should be barking;... [and] the life-cycle explanation is problematic because too many 'old dogs' do in fact bark...41.4\% of issuances and 52.5\% of SEO proceeds come from current and former dividend payers, firms that are clearly beyond the growth phase of their life-cycles." Our theory of SEOs is consistent with findings in both papers. In particular, it can explain why so many "old and young dogs" bark, as it accounts for both offerings for survival and offerings for growth. ${ }^{2}$

A striking new prediction of our theory (from our simulation analysis) is that the equity stake given up to outside investors via an SEO is substantially smaller on average for firms that use the SEO proceeds to invest than for those that do not invest. Such a prediction cannot be obtained in models without persistent productivity shocks.

From a macro perspective, our model sheds light on a major challenge for the realoptions based models that seek to explain the persistently low post-crisis investment despite the substantial increase in corporate cash holdings and the quick recovery of earnings and macroeconomic fundamentals. Several studies, in particular, Baker, Bloom and Davis (2016), and Bloom, Floetotto, Jaimovich, Saporta-Eksten, and Terry (2016), have pointed out that, although investment timing optionality as in Bloom (2009) is highly relevant for understanding investment dynamics, the standard real options models predict only a short-lived pause in investment caused by higher uncertainty following the crisis.

By construction, the standard real options model also cannot explain the increase in corporate savings. Our model with external financing costs, however, produces both more persistent investment dynamics and cash build-ups following a crisis, consistent with the findings in Alfaro, Bloom, and Lin (2016) and Chen (2017). Our model is also consistent with Campello, Graham, and Harvey (2010) and Campello, Giambona, Graham, and Harvey (2011) who find that the more financially constrained firms planned deeper cuts in investment, spending, burned more cash, drew more credit from banks, and also engaged in more asset sales and forced liquidations

\footnotetext{
${ }^{2}$ Carlson, Fisher, and Giammarino $(2006,2010)$ offer a related dynamic theory of SEOs and investment. They assume that investment is always financed through an SEO, and therefore they cannot explain the empirical findings of DeAngelo, DeAngelo and Stulz (2010).
} 
in the crisis. ${ }^{3}$ In sum, our real options model with financial constraints that ties real optionality with financial flexibility predicts significantly more plausible investment and cash dynamics.

Related literature. Following McDonald and Siegel (1986) the basic formulation of the classical real-options problem has been extended in many different directions. Dixit (1989) uses the real option approach to examine entry and exit from a productive activity. Titman (1985) and Williams (1991) analyze real estate development in a real options framework. Abel and Eberly (1994) analyze a unified framework of investment under uncertainty that integrates the $q$ theory of investment with the real options approach.

Mauer and Triantis (1994) considers a real options problem for a levered firm, which otherwise does not face any external financing costs. Hackbarth and Mauer (2012) and Sundaresan, Wang, and Yang (2015) study the joint investment and financing decisions by building on the capital structure model of Leland (1994) and integrating it into the real options framework with institutional features. Grenadier (1996), Lambrecht and Perraudin (2003) and others extend the real options decision problem to a game-theoretic environment. Grenadier and Wang (2005) incorporate informational asymmetries and agency problems into a real options framework. Morellec (2004) and Lambrecht and Myers (2007, 2008) consider managerial agency problems in the context of a real options framework. Kyle, Ou-Yang, and Xiong (2006) introduce prospect theory and Grenadier and Wang (2007) introduce hyperbolic discounting into the classical real options framework.

In strategic dynamic contexts, Grenadier and Malenko (2011) study games in which the decision to exercise an option is a signal of private information to outsiders. Grenadier, Malenko, and Malenko (2016) consider a problem where an uninformed principal makes a timing decision interacting with an informed but biased agent. Orlov, Skrzypacz, and Zryumov (2019) study a Bayesian persuasion game in the context of real options. Miao and Wang (2007) analyze an incomplete-markets realoptions problem. ${ }^{4}$ Grenadier and Malenko (2010) develop a model of real options

\footnotetext{
${ }^{3}$ Ivashina and Scharfstein (2010) also document the aggressive credit-line drawdown by firms in the crisis.

${ }^{4}$ Chen, Miao, and Wang (2010) and Wang, Wang, and Yang (2012) use the incomplete-marketsbased real-options model to study entrepreneurial firms' capital structure, investment, and abandonment decisions. Miao and Wang (2011) analyze the impact of ambiguity and ambiguity aversion
} 
with learning about the permanence of shocks.

None of these models discussed above allow for financial flexibility through the accumulation of corporate savings. More recently, two related articles have introduced financial flexibility into a real options problem, but they only consider onedimensional problems by removing earnings fundamentals as a state variable. The first is by Décamps and Villeneuve (2007) and the second is by Hugonnier, Malamud, and Morellec (2015). Both articles consider a financially constrained firm with an asset in place that generates cash-flows with (transitory) i.i.d shocks and that faces a growth option that can increase the drift of the cash-flow process. Put differently, in the MM version of these models there is no value of waiting and hence there is no real flexibility: the firm would exercise its growth option as soon as it is available.

Thus, our main contribution is to consider a two-dimensional problem that lends itself to a general analysis of the value of real and financial flexibility. Our paper is not the first to consider a two-dimensional real-options problem, but it is the first to offer a complete and rigorous analysis of this problem. The first article to consider a two-dimensional real options problem for a financially constrained firm is by Boyle and Guthrie (2003). They assume that the firm cannot invest at all unless it meets an exogenous borrowing constraint. Once the investment is made the firm is immediately liquidated at the market value of the asset. The main point of their analysis is to highlight an over-investment incentive, which is driven by the risk of (locally) unbounded large losses from the operating assets in place, and the firm's concern that it might be short of cash before it can invest. This concern is contrived as it arises from the ad hoc assumption that the firm is not allowed to voluntarily downsize its operating asset. ${ }^{5}$ That is, they do not account for the firm's abandonment option, which is an important part of our analysis. Unlike their setup, our model fully captures both real and financial flexibility by separating financing and investment option decisions. The firm's financing needs reflect the joint considerations for investment, continued operations, and survival. Moreover, the firm continues to face operating risk after investment and therefore also faces an abandonment option.

Anderson and Carverhill (2012) study a liquidity management problem for a firm

on (real options) investment decisions.

${ }^{5} \mathrm{~A}$ related ad hoc assumption which drives the overinvestment result in their paper is that the growth option payoff value is assumed to follow a geometric Brownian motion, while cumulative operating cash-flows from the operating asset are assumed to follow an arithmetic Brownian motion causing unbounded large operating losses. 
operating over an exogenously fixed time horizon and that is subject to mean-reverting productivity shocks. Besides these different model specifications the other fundamental difference with our analysis is that their model has a one-time investment opportunity at an exogenously fixed time implying no investment-timing flexibility.

Three independent studies analyze a similar problem to ours: First, Copeland and Lyasoff (2013) consider a somewhat narrower framework to ours and do not allow for either abandonment or sequential growth options. Second, Boot and Vladimirov (2014) consider a financially constrained entrepreneurial firm with an asset in place that generates random cash flows following a geometric Brownian motion and a new investment opportunity. Third, Babenko and Tserlukevich (2013) consider the optimal hedging policy for a financially constrained firm with a decreasing returns to scale technology and growth opportunities.

Our paper is also related to Décamps, Mariotti, Rochet, and Villeneuve (2011), who consider a financially constrained firm's optimal dynamic payout and SEO policies, and Bolton, Chen, and Wang $(2011,2013)$, who develop a $q$-theory of investment for financially constrained firms facing i.i.d. shocks. One important difference with the generalized $q$-theory of investment (with convex adjustment costs) is that the financially constrained firm issues equity only for survival and never for investment as it uses its internal savings to smooth investment, and hence cannot explain the above mentioned empirical evidence on SEOs. Bolton, Wang, and Yang (2019) use the recursive optimal contracting method to develop a dynamic liquidity and risk management model also with real investment options for a firm run by an entrepreneur with inalienable human capital.

\section{Model}

Operating Revenues and Profits. We consider a young firm that has assets in place and an investment opportunity. At any point in time $t \geq 0$ the firm can exercise this investment opportunity by paying a fixed investment cost $I>0$ as in McDonald and Siegel (1986). Upon exercising this investment option the young (growth) firm becomes a mature firm. The investment opportunity is more or less attractive depending on the earnings fundamentals $Y$ for its product, which follows the geometric Brownian motion (GBM) process

$$
d Y_{t}=\mu Y_{t} d t+\sigma Y_{t} d B_{t}
$$


where $B_{t}$ is the standard Brownian motion defined on the filtered probability space $\left(\Omega, \mathcal{F},\left\{\mathcal{F}_{t}\right\}_{t \in \mathbb{R}_{+}}, \mathbb{P}\right)$ which satisfies the usual conditions.

The firm generates revenues at the rate of $m_{n} Y$, where $m_{n}$ denotes the firm's production capacity, with $n \in\{1,2\}$. When $n=1$ the firm is in the growth phase, and when $n=2$ the firm has expanded into its mature phase, so that $m_{1}<m_{2}$. We denote the firm's deterministic operating cost (per unit time) by $Z_{n}>0$, so that the operating profit per unit of time is $\left(m_{n} Y_{t}-Z_{n}\right)$. Obviously, our model specification allows both the revenue and cost to be stochastic and only requires that the operating profit $\left(m_{n} Y-Z_{n}\right)$ is an affine function in $Y$. In sum, upon exercising the growth option the firm's operating revenue increases by $\Delta_{m} Y_{t}$, where $\Delta_{m}=m_{2}-m_{1}$, and its operating cost increases by $\Delta_{Z}=Z_{2}-Z_{1}$, so that $\left(\Delta_{m} Y_{t}-\Delta_{Z}\right)$ is the firm's incremental profit from the second asset.

When profits $\left(m_{n} Y_{t}-Z_{n}\right)$ are sufficiently negative it may be optimal for the firm to abandon operations as the option value of continuing the firm is no longer positive. As in the standard real-options literature (e.g., McDonald and Siegel, 1986), by exercising the abandonment option, the firm shuts itself down and without loss of generality obtains a liquidation value of zero. The firm, thus, has an American-style perpetual liquidation option, where the timing of the option is endogenously chosen in both the growth and mature phases. In sum, our model describes a simple life-cycle of a firm with three phases: a growth phase, a mature phase, and a liquidation (or scale-down) phase. We assume that investors are risk neutral, so that all cash flows are discounted at the risk-free rate $r$. Equivalently, we may interpret the process (1) as representing the risk-neutral measure.

External Financing Costs. In reality firms often face significant external financing costs due to asymmetric information and managerial incentive issues. A large empirical literature has sought to measure these costs, in particular the costs arising from the negative stock price reaction in response to the announcement of a new equity issue. ${ }^{6}$

\footnotetext{
${ }^{6}$ An early study by Asquith and Mullins (1986) found that the average stock price reaction to the announcement of a common stock issue was $-3 \%$ and the loss in equity value as a percentage of the size of the new equity issue was as high as $-31 \%$. Similar results have been obtained in several subsequent studies (see Eckbo, Masulis and Norli, 2007 for a survey). Calomiris and Himmelberg (1997) estimate the direct transactions costs firms face when they issue equity and find that mean transactions costs (underwriting, management, legal, auditing and registration fees) are as high as $9 \%$ of an issue for seasoned public offerings and $15.1 \%$ for initial public offerings.
} 
To capture these costs, we use a standard specification for external financing costs. $^{7}$ If the firm needs to raise external funds $F$ net of fees, it incurs an external financing cost:

$$
\Phi(F)=\phi_{0} \mathcal{I}_{F>0}+\phi_{1} F,
$$

where $\phi_{0} \geq 0$ is the fixed cost parameter and $\phi_{1} \geq 0$ is the marginal cost of external financing, and $\mathcal{I}_{F>0}$ is an indicator function which takes the value of one when $F>0$ and zero otherwise. ${ }^{8}$ When the fixed $\operatorname{cost} \phi_{0}$ is high the firm may prefer liquidation over equity issuance.

While we could allow for different equity issuance costs in the growth and mature phases to capture different types of financing frictions (agency costs and informational asymmetry), we keep the external financing costs the same in both phases for simplicity. An important simplification, however, is that we do not explicitly model informational asymmetries between the firm and outside investors. As a result our model cannot capture how investment and abandonment policies differ across different types of issuers and how asymmetric information about option values maps into external financing costs.

Corporate Liquidity Management. To avoid incurring external financing costs too often the firm will seek to accumulate funds internally, so that a key aspect of the firm's financial management is liquidity, or cash, management, to which we next turn.

The growth phase. At the beginning of the growth phase $(t=0)$ the firm is endowed with a stock of cash $W_{0} \geq 0$. Over time the firm's cash accumulates as follows

$$
d W_{t}=\left(r W_{t}+m_{1} Y_{t}-Z_{1}\right) d t+d C_{t}-d U_{t},
$$

subject to the constraint that its cash holding is non-negative:

$$
W_{t} \geq 0 \text {. }
$$

The first term in (3) is the sum of the firm's interest income $r W$ and operating profits $m_{1} Y_{t}-Z_{1}$. The second term in (3), $C_{t}$, denotes the firm's non-decreasing

\footnotetext{
${ }^{7}$ Explicitly modeling informational asymmetry would result in a substantially more involved analysis. Lucas and McDonald (1990) provides a tractable analysis by making the simplifying assumption that the informational asymmetry is short lived, i.e. it lasts one period.

${ }^{8}$ In other words, to raise the net amount $F$ the firm must raise the gross amount $F+\Phi(F)$ from investors.
} 
cumulative external equity financing, and the third term $U_{t}$ denotes its non-decreasing cumulative payout, in that $d C_{t} \geq 0$ and $d U_{t} \geq 0$. Should the firm choose to abandon its operations, it then pays out any remaining cash $W_{t}$ to its shareholders. We begin by considering this simpler situation, and later extend the analysis to situations where the firm incurs a cash-carrying cost and therefore must also determine its payout policy.

When the firm's liquidity $W$ is insufficient to cover the investment cost, $W<I$, the firm will obviously have to raise external funds to finance the investment cost $I$ or alternatively the firm can wait and continue to accumulate internal funds. This is one way in which external financing costs will modify the firm's exercise decision of its growth option. Also, when the firm chooses to raise external funds to finance its investment it may decide to raise more funds than needed to cover the investment cost because it also needs funds (working capital) to finance potential operating losses after it has exercised its growth option.

The mature phase. In the mature phase cash accumulates as follows:

$$
d W_{t}=\left(r W_{t}+m_{2} Y_{t}-Z_{2}\right) d t+d C_{t}-d U_{t}, \quad W_{t} \geq 0
$$

In other words, the dynamics of liquidity are almost the same as in the growth phase, the only difference being that production capacity is now $m_{2}$ and operating costs are $Z_{2}$.

The Optimization Problem. For expositional purposes, we denote by $\left\{A_{t}\right\}_{t \in \mathbb{R}_{+}}$ the control process which takes the liquidity process into the positive closed region $\left\{W_{t} \geq 0\right\}_{t \in \mathbb{R}_{+}}$.

Let $\tau_{L}^{n}$ denote the abandonment (liquidation) time in the growth phase $(n=1)$ and in the mature phase $(n=2)$. Also let $\tau_{i}$ denote the stochastic investment time in the growth phase. Next, we turn to the stochastic equity issuance timing and amount. Let $\tau_{F}^{n}(t)$ denote the first external financing time after time $t$ in phase $n$, where $n=1$ for the growth phase and $n=2$ for the mature phase, and let $F_{n} \geq 0$ denote the corresponding external financing amount at time $\tau_{F}^{n}(t)$.

As the firm's discount rate is equal to the risk-free rate, there is no cost for the firm to carry cash in the firm, which means that it is weakly efficient for shareholders to save the firm's profits inside the firm. It is possible that the firm's savings at time $t, W_{t}$, is so high that the firm becomes permanently financially unconstrained at all 
its future time $s \geq t$. That is, it is optimal for the firm to choose the first-best policies at all $s \geq t$ with probability one. We use $\tau_{U}^{1}$ to denote the (first) time $t$ when the firm becomes permanently financially unconstrained in the growth phase. Similarly, we use $\tau_{U}^{2}$ to denote the (first) time when the firm becomes permanently financially unconstrained in the mature phase.

The control process is then given by $A_{t} \equiv\left(\tau_{U}^{2}, \tau_{L}^{2}, \tau_{F}^{2}(t), F_{2}\right)$ in the mature phase and by $A_{t} \equiv\left(\tau_{i}, \tau_{U}^{1}, \tau_{L}^{1}, \tau_{F}^{1}(t), F_{1}\right)$ in the growth phase. ${ }^{9}$ The extra control in the growth phase is due to the investment option.

Before defining the value function and providing its solution, we state the following assumptions.

Assumption 1 There exists a nonempty compact metric space $\mathcal{K}$ such that $A_{t} \in \mathcal{K}$ for all $t \in \mathbb{R}_{+}$.

A control process is said to be admissible if: 1) it is adapted to the filtration $\left.\left\{\mathcal{F}_{t}\right\}_{t \in \mathbb{R}_{+}} ; 2\right)$ it satisfies Assumption 1; and, 3) $W_{t}$ following the process, defined by equation (3) for the growth phase and the process defined by equation (5) for the mature phase, has a unique strong solution. The set of admissible control processes is denoted by $\mathcal{A}$.

Next, we state a sufficient condition that ensures that the value function is well defined.

Assumption 2 The risk-free interest rate is positive, $r>0$, and the growth rate of the earnings fundamentals is strictly smaller than the risk-free interest rate, $r>\mu$.

Finally, to ensure that the investment option is valuable in the growth phase, we impose the following assumption.

Assumption 3 The firm's production capacity in the mature phase is strictly greater than that in growth phase: $m_{2}>m_{1}$.

In sum, the firm's optimization problem involves several dynamic policies: i) an investment timing decision; ii) an abandonment timing decisions; and iii) dynamic SEO decisions. Importantly, the firm uses liquidity management to integrate these interdependent decisions.

\footnotetext{
${ }^{9}$ For notational simplicity, sometimes we skip time subscripts of the control process. For example, $A$ refers to $A_{t}$ and $\tau_{F}^{n}(t)$ refers to $\tau_{F}^{n}$.
} 


\section{The First-Best Solution}

Under perfect capital markets (when the Modigliani-Miller theorem holds), the firm only faces investment and abandonment timing decisions. We solve for the firm's value in both mature and growth phases together with its optimal abandonment and investment policies.

First, we calculate the firm's value (i.e. from its asset in place) in the mature phase. We use $Q^{*}(Y)$ to denote its value in the mature phase. The only decision in the mature phase for the fist-best $(\mathrm{FB})$ setting is the abandonment timing decision. The firm's value is given by

$$
Q^{*}\left(Y_{t}\right)=\max _{\tau_{L}^{2}} \mathbb{E}_{t}\left[\int_{t}^{\tau_{L}^{2}} e^{-r(s-t)}\left(m_{2} Y_{s}-Z_{2}\right) d s\right]
$$

This equation incorporates the value of abandonment as the integral is truncated at the liquidation time $\tau_{L}^{2}$.

Next, we calculate the firm's value in the growth phase where it has one asset in place and a growth option. We use $H^{*}(Y)$ to denote its value in the growth phase. The decisions in the growth phase for the FB setting then involve both abandonment and investment timing decisions. Therefore, the firm's value is then given by

$$
H^{*}\left(Y_{t}\right)=\max _{\tau_{i}, \tau_{L}^{1}} \mathbb{E}_{t}\left[\int_{t}^{\min \left\{\tau_{L}^{1}, \tau_{i}\right\}} e^{-r(s-t)}\left(m_{1} Y_{s}-Z_{1}\right) d s+e^{-r\left(\tau_{i}-t\right)}\left(Q^{*}\left(Y_{\tau_{i}}\right)-I\right)\right] .
$$

As the optimal timing decisions are characterized by optimal threshold policies in our model, we next introduce various threshold policies. We use $Y_{a, 2}^{*}$ to denote the optimal abandonment hurdle in the mature phase: $\tau_{L}^{2}=\inf \left\{s \mid Y_{s} \leq Y_{a, 2}^{*}, s \geq t\right\}$, use $Y_{a, 1}^{*}$ to denote the optimal abandonment threshold in the growth phase: $\tau_{L}^{1}=$ $\inf \left\{s \mid Y_{s} \leq Y_{a, 1}^{*}, s \geq t\right\}$, and use $Y_{i}^{*}$ to denote the optimal investment threshold: $\tau_{i}=\inf \left\{s \mid Y_{s} \geq Y_{i}^{*}, s \geq t\right\}$.

The following theorem states the first-best solution under perfect capital markets.

Theorem 1 Under Assumptions 1-2, the value of the operating asset $Q^{*}(Y)$ in the mature phase is given by:

$$
Q^{*}(Y)=\left(\frac{m_{2} Y}{r-\mu}-\frac{Z_{2}}{r}\right)-\left(\frac{m_{2} Y_{a, 2}^{*}}{r-\mu}-\frac{Z_{2}}{r}\right)\left(\frac{Y}{Y_{a, 2}^{*}}\right)^{\gamma} \quad \text { for } \quad Y \geq Y_{a, 2}^{*},
$$


where the abandonment hurdle $Y_{a, 2}^{*}$ is given by (A.1) and the constant $\gamma$ is given by (A.2) in Appendix A.

Under Assumptions 1-3, the firm's value in the growth phase, $H^{*}(Y)$, is given by:

$$
\begin{aligned}
H^{*}(Y)= & \frac{m_{1} Y}{r-\mu}-\frac{Z_{1}}{r}+\frac{\left(Y_{a, 1}^{*}\right)^{\gamma} Y^{\beta}-\left(Y_{a, 1}^{*}\right)^{\beta} Y^{\gamma}}{\left(Y_{a, 1}^{*}\right)^{\gamma}\left(Y_{i}^{*}\right)^{\beta}-\left(Y_{a, 1}^{*}\right)^{\beta}\left(Y_{i}^{*}\right)^{\gamma}}\left(Q^{*}\left(Y_{i}^{*}\right)-I-\left(\frac{m_{1} Y_{i}^{*}}{r-\mu}-\frac{Z_{1}}{r}\right)\right) \\
& -\frac{\left(Y_{i}^{*}\right)^{\beta} Y^{\gamma}-\left(Y_{i}^{*}\right)^{\gamma} Y^{\beta}}{\left(Y_{a, 1}^{*}\right)^{\gamma}\left(Y_{i}^{*}\right)^{\beta}-\left(Y_{a, 1}^{*}\right)^{\beta}\left(Y_{i}^{*}\right)^{\gamma}}\left(\frac{m_{1} Y_{a, 1}^{*}}{r-\mu}-\frac{Z_{1}}{r}\right), \quad \text { for } \quad Y_{a, 1}^{*} \leq Y \leq Y_{i}^{*}
\end{aligned}
$$

where the constant $\gamma$ and $\beta$ are given by (A.2) and (A.5) respectively, and the investment threshold $Y_{i}^{*}$ and abandonment threshold $Y_{a, 1}^{*}$ jointly solve the equations (A.3) and (A.4) in Appendix A.

Moreover, $H^{*}(Y)=Q^{*}(Y)-I$ for $Y>Y_{i}^{*}$, and $H^{*}(Y)=0$ for $Y<Y_{a, 1}^{*}$.

For the mature phase, the value of the firm's assets in place is equal to $Q^{*}(Y)$. The first term in (8) for $Q^{*}(Y)$ is the present discounted value of operating profits if the firm were to remain in operation forever (which would be suboptimal for sufficiently low $Y$ ). The second term in (8) for $Q^{*}(Y)$ is the abandonment option value. The firm operates its asset if and only if $Y \geq Y_{a, 2}^{*}$. When $Y<Y_{a, 2}^{*}$ the asset is abandoned and $Q^{*}(Y)=0$. As is well known, $Q^{*}(Y)$ is convex in $Y$ due to the abandonment option.

For the growth case, the enterprise value is equal to $H^{*}(Y)$. The first term in (9) for $H^{*}(Y)$ is the present discounted value of operating profits if the firm were to remain in operation forever generating profits at the rate of $m_{1} Y-Z_{1}$ without exercising either abandonment or growth option. The second term in (9) for $H^{*}(Y)$ is the investment option value (taking into account that the growth option is exercised before the abandonment is exercised in the growth phase.) The third term givens the value of the abandonment in the growth phase (taking into account that the firm is optimally abandoned before it exercises its growth option.)

The optimal threshold for the abandonment option in the mature phase, $Y_{a, 2}^{*}$, has the standard well-known formula given by (A.1). The optimal threshold for the abandonment option in the growth phase and that for the growth option are determined jointly by (A.3) and (A.4).

The proof for Theorem 1 is standard and omitted. Dayanik and Karatzas (2003) provide a general characterization of one-dimensional optimal-stopping problems. 
Finally, we note that under MM, in the mature phase, the value of a firm, denoted by $P^{*}(W, Y)$, is simply equal to the sum of its cash holdings $W$ and the value of its asset in place, $Q^{*}(Y)$, i.e.,

$$
P^{*}(W, Y)=Q^{*}(Y)+W .
$$

Similarly, in the growth phase the firm's value, denoted by $G^{*}(W, Y)$, is given by

$$
G^{*}(W, Y)=H^{*}(Y)+W .
$$

The functions, $G^{*}(W, Y)$ and $P^{*}(W, Y)$, are the upper bounds for the firm's value in the growth and mature phase, respectively.

\section{Solution: General Case with External Financing Costs}

When the firm faces external financing costs it will optimally hoard liquidity $W$ in order to reduce the frequency with which it returns to capital markets to raise external funds. The interaction between earnings fundamentals and corporate liquidity gives rise to highly nonlinear dynamics for firm value, investment, and financing.

\subsection{The Mature Phase}

We begin by defining the liquidity threshold $\Lambda_{2}$ in the mature phase such that the firm is permanently financially unconstrained for any liquidity beyond that threshold.

Definition 1 Let $\Lambda_{2}$ denote the liquidity threshold for $W$ such that:

$$
\Lambda_{2}=\frac{r-\gamma \mu}{r^{2}(1-\gamma)} Z_{2},
$$

and $\tau_{U}^{2}$ denote the first time $W_{t}$ hits $\Lambda_{2}$ in mature phase: $\tau_{U}^{2}=\inf \left\{s \mid W_{s} \geq \Lambda_{2}, s \geq t\right\}$. Furthermore, let $\tau_{2}=\min \left\{\tau_{F}^{2}, \tau_{L}^{2}, \tau_{U}^{2}\right\}$.

Given an admissible control $A$, the value function in the mature phase is given by:

$$
\begin{array}{r}
P\left(W_{t}, Y_{t} ; A\right)=\mathbb{E}_{t}\left[e^{-r\left(\tau_{2}-t\right)}\left[P\left(W_{\tau_{2}-}+F_{2}, Y_{\tau_{2}-}\right)-F_{2}-\Phi\left(F_{2}\right)\right] \mathcal{I}_{\tau_{2}=\tau_{F}^{2}}\right. \\
\left.+e^{-r\left(\tau_{2}-t\right)} W_{\tau_{2}} \mathcal{I}_{\tau_{2}=\tau_{L}^{2}}+e^{-r\left(\tau_{2}-t\right)} P^{*}\left(W_{\tau_{2}}, Y_{\tau_{2}}\right) \mathcal{I}_{\tau_{2}=\tau_{U}^{2}}\right] .
\end{array}
$$


Equation (13) takes into account the three mutually exclusive scenarios. First, the firm issues costly external equity at $\tau_{F}^{2}$, captured by the first term when $\tau_{2}=\tau_{F}^{2}$. Second, the firm liquidates, captured by the second term when $\tau_{2}=\tau_{L}^{2}$. Third, the firm becomes permanently financially unconstrained, captured by the last term when $\tau_{2}=\tau_{U}^{2}$. As the firm is permanently financially unconstrained at $\tau_{U}^{2}$, the firm attains the first-best mature value $P^{*}\left(W_{t}, Y_{t}\right)$ at $t=\tau_{U}^{2}$.

Assumption 2 guarantees that the value function $P\left(W_{t}, Y_{t} ; A\right)$ is well defined for any admissible control. The value function in the mature phase, denoted $P\left(W_{t}, Y_{t}\right)$, is then given by

$$
P\left(W_{t}, Y_{t}\right)=\sup _{A \in \mathcal{A}} P\left(W_{t}, Y_{t} ; A\right) .
$$

An admissible control is said to be optimal if $P\left(W_{t}, Y_{t}\right)=P\left(W_{t}, Y_{t} ; A\right)$.

As a first step in the characterization of $P(W, Y)$, we state the following lemmas that summarize the main properties of $P(W, Y)$.

Lemma 1 The value function $P(W, Y)$ must be greater than or equal to the firm's liquidation value at any time, that is:

$$
P(W, Y) \geq W
$$

Lemma 1 follows from the fact that the firm can always liquidate itself and collect $W$. The (net) value of the perpetual American liquidation option, measured by $P(W, Y)-W$ is weakly positive.

Lemma 2 The firm always has an option to issue equity, so that $P(W, Y)$ satisfies

$$
P(W, Y) \geq P^{F}(W, Y)
$$

where $P^{F}(W, Y)$ would be the firm's value if the firm were to immediately issue equity:

$$
P^{F}(W, Y)=\sup _{F_{2} \geq 0} P\left(W+F_{2}, Y\right)-F_{2}-\Phi\left(F_{2}\right) .
$$

Similar to our argument for Lemma 1, Lemma 2 follows from the fact that the firm can always issue equity at a cost. By revealed preferences, the fact that the firm chooses not to issue equity must imply that the timing is not optimal and hence the inequality given in (16) has to hold. 
Lemma 3 The firm's value $P(W, Y)$ attains the first-best value when $W \geq \Lambda_{2}$ :

$$
P(W, Y)=P^{*}(W, Y)=W+Q^{*}(Y), \quad \text { for } \quad W \geq \Lambda_{2}
$$

Lemma 3 establishes that the firm is permanently financially unconstrained when its liquidity $W$ exceeds $\Lambda_{2}$, which is given by (12).

The reasoning for this result is as follows. As long as the firm's savings rate, $\left(r W+m_{2} Y-Z_{2}\right)$, is non-negative, when evaluated at the firm's first-best abandonment threshold $Y_{a, 2}^{*}$, i.e., $\left(r W+m_{2} Y-Z_{2}\right) \geq 0$, the firm is never involuntarily liquidated, can always achieve the first-best policies, and hence is permanently financially unconstrained. The preceding inequality is equivalent to

$$
W \geq \Lambda_{2}
$$

where $\Lambda_{2}$ is obtained by substituting the explicit formula for the first-best abandonment hurdle $Y_{a, 2}^{*}$, given in (A.1), into $\left(r W+m_{2} Y-Z_{2}\right) \geq 0$ :

$$
\Lambda_{2}=\int_{t}^{\infty} e^{-r(s-t)}\left(Z_{2}-m_{2} Y_{a, 2}^{*}\right) d s=\frac{Z_{2}-m_{2} Y_{a, 2}^{*}}{r}=\frac{r-\gamma \mu}{r^{2}(1-\gamma)} Z_{2} .
$$

In sum, the firm is financially unconstrained only when it faces no financial constraint with probability one at the current and all future times. This is the case when $W \geq \Lambda_{2}$, where $\Lambda_{2}$ denotes the lowest level of liquidity needed for a mature firm to be permanently financially unconstrained. ${ }^{10}$

The firm's value $P(W, Y)$ is then equal to the first-best value:

$$
P(W, Y)=P^{*}(W, Y)=W+Q^{*}(Y) \text { for } \quad W \geq \Lambda_{2}
$$

where the first-best value $Q^{*}(Y)$ is given by (8). For a firm to be financially unconstrained, it cannot issue external equity with strictly positive probability at any moment because external funding is costly and distorts corporate decisions. That is, the condition for a firm to be financially unconstrained in a dynamic setting is much tighter than in a static setting.

\footnotetext{
${ }^{10}$ Our notion of minimum liquidity holding $W$ for a financially unconstrained firm is analogous to the notion of natural borrowing limit in the macroeconomics savings literature following Aiyagari (1994). This borrowing limit is defined as the maximum amount of risk-free debt a consumer can accumulate without ever defaulting. A consumer can borrow at the risk-free rate up to that natural borrowing limit, but any additional amount of borrowing will give rise to default risk. For a firm, $\Lambda_{2}$ is the minimum $W$ that it needs in order to implement its first-best abandonment policy. Any liquidity holding lower than $\Lambda_{2}$ may induce under-investment via inefficient liquidation with positive probability.
} 
Lemma 4 In the limit as $Y \rightarrow \infty$, the firm's value $P(W, Y)$ attains the first-best value, that is

$$
P(W, Y)=P^{*}(W, Y)=W+Q^{*}(Y), \quad \text { for } \quad Y \rightarrow \infty
$$

The reason is that, as its operating revenue $Y \rightarrow \infty$, the firm is permanently financially unconstrained. Therefore, the firm can support all its first-best policy with probability one and hence attains the maximal value under the first-best.

Next, we make the following assumption.

Assumption 4 The function, $P(W, Y)$, is smooth in the inaction region.

The function, $P(W, Y)$, then satisfies $\mathcal{L}_{2} P=0$, where $\mathcal{L}_{2}$ is the infinitesimal generator given by

$$
\mathcal{L}_{2} P=\left(r W+m_{2} Y-Z_{2}\right) P_{W}+\mu Y P_{Y}+\frac{\sigma^{2} Y^{2}}{2} P_{Y Y}-r P .
$$

The condition $\mathcal{L}_{2} P=0$ follows from the standard principle of dynamic optimality. The first term on the right side of (23), which is given by the product of the firm's marginal value of cash $P_{W}(W, Y)$ and its saving rate $\left(r W+m_{2} Y-Z_{2}\right)$, captures the value of the firm's savings. The second term, $\mu Y P_{Y}$, represents the marginal effect of an expected revenue change $\mu Y$ on firm value, and the third term in $P_{Y Y}$ encapsulates the effects of the volatility of changes in earnings fundamentals $Y$ on firm value. Intuitively, in the region where the firm operates normally the sum of the first three terms, which amount to the total expected change in firm value $P(W, Y)$, must equal $r P(W, Y)$ the normalized value of the firm.

When the firm issues equity or liquidates itself, in other words when either (15) or (16) binds, $P(W, Y)$ satisfies

$$
\mathcal{L}_{2} P \leq 0
$$

We summarize the model solution for the mature phase in the following theorem.

Theorem 2 Under Assumptions 1, 2, and 4, the value function in the mature phase, $P(W, Y)$, can be solved via the variational-inequality formulation for $(W, Y) \in \Sigma_{2}$, where $\Sigma_{2}=\left\{(W, Y) \mid 0 \leq W \leq \Lambda_{2}, 0<Y<\infty\right\}$ denotes the solution region:

$$
\max \left\{\mathcal{L}_{2} P, \max \left\{P^{F}(W, Y), W\right\}-P(W, Y)\right\}=0
$$


where $P^{F}(W, Y)$ is defined by (17). Additionally, the following boundary condition holds

$$
P(W, Y)=P^{*}(W, Y)=W+Q^{*}(Y), \quad \text { for } \quad W=\Lambda_{2} \quad \text { or } \quad Y \rightarrow \infty
$$

The intuition for (25) is as follows. If the firm is in the inaction region, $\mathcal{L}_{2} P=0$, which characterizes the solution, has to hold. Otherwise, the firm must take one of the following two actions: (1) issuing equity to replenish liquidity or (2) liquidating itself and returning all cash to its shareholders, as one of the two inequalities, (16 or (15), must be satisfied with equality at the boundary, which implies $P(W, Y)=$ $\max \left\{P^{F}(W, Y), W\right\}$ at the boundary. Taking the boundary behaviors together with the inaction option, we conclude that (25) has to hold.

Next, we establish several fundamental properties of the value function $P(W, Y)$.

Proposition 1 Under Assumptions 1-2 and 4, the following properties for $(W, Y) \in$ $\Sigma_{2}$ hold:

i) the solution for $P(W, Y)$ exists and is bounded as $W \leq P(W, Y) \leq P^{*}(W, Y)$;

ii) the solution for $P(W, Y)$ is strictly increasing in $W$ and strictly increasing in $Y$ before liquidation. Additionally, $Q(W, Y)=P(W, Y)-W$ is increasing in $W$;

iii) the solution for $P(W, Y)$ is unique.

Finally, we establish the following properties for the liquidation and external financing decisions.

Proposition 2 Under Assumptions 1-2, i) the optimal liquidation boundary, denoted by $\underline{Y}_{2}(W)$, is decreasing in $W$; ii) the firm will delay costly equity issuance until it has entirely exhausted its cash.

The proofs for the results in this subsection are provided in Appendix B.

We next turn to the analysis for the growth phase. Anticipating its potential financial constraints in the mature phase, a rational forward-looking firm takes these future constraints into account for its decisions in the growth phase.

\subsection{The Growth Phase}

In the growth phase the firm faces an optimal investment timing problem along with abandonment timing and external equity financing decisions. 
Definition 2 Let $\Lambda_{1}$ denote the liquidity threshold given by

$$
\Lambda_{1}=\frac{Z_{1}-m_{1} Y_{a, 1}^{*}}{r}
$$

where $Y_{a, 1}^{*}$ is the first-best abandonment hurdle in the growth phase. Let $\tau_{U}^{1}$ denote the first time liquidity reaches $\Lambda$ in the growth phase, i.e., $\tau_{U}^{1}=\inf \left\{s \mid W_{s} \geq \Lambda\right\}$, where $\Lambda$ is given by

$$
\Lambda=\max \left\{\Lambda_{1}, \Lambda_{2}+I\right\} .
$$

Finally, let $\tau_{1}=\min \left\{\tau_{i}, \tau_{F}^{1}, \tau_{L}^{1}, \tau_{U}^{1}\right\}$.

Note that $\tau_{U}^{1}$ is the first time when the firm has accumulated sufficient liquidity to be permanently unconstrained and hence it attains the first-best firm value $G^{*}\left(W_{t}, Y_{t}\right)$ with probability one under all circumstances. Because the firm is forward looking, the amount of liquidity it is required for the firm to never compromise its investment and liquidation decisions is to have at least the level of liquidity exceeding $\max \left\{\Lambda_{1}, \Lambda_{2}+I\right\}$.

Given an admissible control $A$, the value function in the growth phase is given by

$$
\begin{aligned}
G\left(W_{t}, Y_{t} ; A\right)=\mathbb{E}_{t}\left[e^{-r\left(\tau_{1}-t\right)}\left[P\left(W_{\tau_{1}-}+F_{1}-I, Y_{\tau_{1}-}\right)-F_{1}-\Phi\left(F_{1}\right)\right] \mathcal{I}_{\tau_{1}=\tau_{i}}\right. \\
+e^{-r\left(\tau_{1}-t\right)}\left[G\left(W_{\tau_{1}-}+F_{1}, Y_{\tau_{1-}}\right)-F_{1}-\Phi\left(F_{1}\right)\right] \mathcal{I}_{\tau_{1}=\tau_{F}^{1}} \\
\left.+e^{-r\left(\tau_{1}-t\right)} W_{\tau_{1}} \mathcal{I}_{\tau_{1}=\tau_{L}^{1}}+e^{-r\left(\tau_{1}-t\right)} G^{*}\left(W_{\tau_{1}}, Y_{\tau_{1}}\right) \mathcal{I}_{\tau_{1}=\tau_{U}^{1}}\right] .
\end{aligned}
$$

Equation (29) takes into account the four mutually exclusive scenarios in the mature phase. First, the firm invests when $\tau_{1}=\tau_{i}$. Second, the firm issues equity and exercises the growth option when $\tau_{1}=\tau_{F}^{1}$. Third, the firm liquidates when $\tau_{1}=\tau_{L}^{1}$. Finally, the firm becomes permanently financially unconstrained when $\tau_{1}=\tau_{U}^{1}$. As the firm is permanently financially unconstrained at $\tau_{U}^{1}$, the firm attains the firstbest value $G^{*}\left(W_{t}, Y_{t}\right)$ at $t=\tau_{U}^{1}$ in the growth phase, which means that the firm can support its growth-option exercising and liquidation decisions at its first-best levels in the future.

Assumptions 2-3 guarantee that the value function is well defined for any admissible control. Similarly, the value function in the growth phase, denoted $G\left(W_{t}, Y_{t}\right)$, is defined by

$$
G\left(W_{t}, Y_{t}\right)=\sup _{A \in \mathcal{A}} G\left(W_{t}, Y_{t} ; A\right) .
$$

An admissible control is said to be optimal if $G\left(W_{t}, Y_{t}\right)=G\left(W_{t}, Y_{t} ; A\right)$.

As for the mature phase, we spell out a number of lemmas that summarize the main properties of $G(W, Y)$. 
Lemma 5 The value function in the growth phase $G(W, Y)$ must be greater than or equal to the firm's liquidation value at any time, that is:

$$
G(W, Y) \geq W
$$

The reasoning for Lemma 5 for the growth phase is the same as that for Lemma 1 for the mature phase: The firm can always liquidate itself and collect $W$ and the (net) value of the perpetual American liquidation option, measured by $G(W, Y)-W$ is always weakly positive.

Lemma 6 The firm always has an option to issue equity in the growth phase, so that $G(W, Y)$ must satisfy

$$
G(W, Y) \geq G^{F}(W, Y)
$$

where $G^{F}(W, Y)$ would be the firm's value if the firm were to immediately issue equity:

$$
G^{F}(W, Y)=\sup _{F_{1} \geq 0} G\left(W+F_{1}, Y\right)-F_{1}-\Phi\left(F_{1}\right)
$$

Similarly, the reasoning for Lemma 6 for the growth phase is the same as that for Lemma 2 for the mature phase: The timing flexibility in issuing equity is valuable in the growth phase and $G(W, Y) \geq G^{F}(W, Y)$.

Lemma 7 The firm always has an option to invest in the growth phase, $G(W, Y)$ must satisfy

$$
G(W, Y) \geq G^{I}(W, Y)
$$

where $G^{I}(W, Y)$ would be the firm's value if the firm were to immediately issue equity to partially finance its growth option exercising:

$$
G^{I}(W, Y)=\sup _{F_{1} \geq 0} P\left(W+F_{1}-I, Y\right)-F_{1}-\Phi\left(F_{1}\right)
$$

Similarly, Lemma 7 implies the firm's flexibility in choosing the timing of its investment option is always valuable in the growth phase.

Lemma 8 The firm's value in the growth phase $G(W, Y)$ attains the first-best value when $W \geq \max \left\{\Lambda_{1}, \Lambda_{2}+I\right\}$ :

$$
G(W, Y)=G^{*}(W, Y)=W+H^{*}(Y), \quad \text { for } \quad W \geq \max \left\{\Lambda_{1}, \Lambda_{2}+I\right\}
$$


Lemma 8 establishes that for the firm to be permanently financially unconstrained in the growth phase it must be able to pursue all its real decisions including investment timing and liquidation (which could occur in either phase) with probability one. This occurs when $W \geq \max \left\{\Lambda_{1}, \Lambda_{2}+I\right\}$. The intuition is as follows. First, to avoid inefficient liquidation in the growth phase, the firm requires at least $\Lambda_{1}$ in its savings account. To avoid inefficient investment and suboptimal liquidation in the mature phase, the firm at least has to have $\Lambda_{2}+I$, out of which the amount of $\Lambda_{2}$ ensures that the firm is willing to choose its liquidation policy at the first-best level and the amount of $I$ ensures that the firm has plenty liquidity to exercise its growth option at the first-best level.

Lemma 9 As $Y \rightarrow \infty$, the firm's value $G(W, Y)$ attains the first-best value, that is

$$
G(W, Y)=G^{*}(W, Y)=W+H^{*}(Y), \quad \text { for } \quad Y \rightarrow \infty
$$

The reasoning for this result is the same as that for Lemma 4. As its operating revenue $Y \rightarrow \infty$, the firm is permanently financially unconstrained. Therefore, the firm can support its first-best investment and liquidation policies with probability one and hence its value attains the first-best level.

Next, we make the following assumption.

Assumption 5 The function, $G(W, Y)$, is smooth in the inaction region.

The function, $G(W, Y)$, then satisfies $\mathcal{L}_{1} G=0$, where $\mathcal{L}_{1}$ is the infinitesimal generator in the growth phase:

$$
\mathcal{L}_{1} G=\left(r W+m_{1} Y-Z_{1}\right) G_{W}+\mu Y G_{Y}+\frac{\sigma^{2} Y^{2} G_{Y Y}}{2}-r G .
$$

When the firm issues equity or liquidates itself, i.e., when either (31), (32), or (34) binds, $G(W, Y)$ satisfies

$$
\mathcal{L}_{1} G \leq 0 .
$$

The following theorem summarizes the model solution for the growth phase.

Theorem 3 Under Assumptions 1-5, the value function in the growth phase, $G(W, Y)$, can be solved via the variational-inequality formulation for $(W, Y) \in \Sigma_{1}$, where $\Sigma_{1}=$ $\left\{(W, Y) \mid 0 \leq W \leq \max \left\{\Lambda_{1}, \Lambda_{2}+I\right\}, 0<Y<\infty\right\}$ denotes the solution region:

$$
0=\max \left\{\mathcal{L}_{1} G, \max \left\{G^{F}(W, Y), G^{I}(W, Y), W\right\}-G(W, Y)\right\}
$$


where $G^{F}(W, Y)$ and $G^{I}(W, Y)$ are defined by (33) and (35), respectively. Additionally, the following boundary condition holds:

$$
G(W, Y)=G^{*}(W, Y)=W+H^{*}(Y), \quad \text { for } \quad W \geq \max \left\{\Lambda_{1}, \Lambda_{2}+I\right\} \quad \text { or } \quad Y \rightarrow \infty .
$$

The intuition for (40) is as follows. If the firm is in the inaction region, $\mathcal{L}_{1} G=0$, which characterizes the solution, has to hold. Otherwise, the firm must take one of the following three actions: (1) issuing equity to replenish liquidity, (2) issuing equity to partially finance the cost of exercising the growth option, or (3) liquidating itself and returning all cash to its shareholders, as one of the three inequalities (32), (34), or (31) must be satisfied with equality at the boundary, which implies $G(W, Y)=$ $\max \left\{G^{F}(W, Y), G^{I}(W, Y), W\right\}$ at the boundary. Taking the boundary behaviors together with the inaction option, we conclude that (40) has to hold.

Depending on its liquidity holding $W$, the firm finds itself in the following three different regions: .

1. Financially Unconstrained Region: $W \geq \max \left\{\Lambda_{1}, \Lambda_{2}+I\right\}$. If $W \geq \Lambda_{1}$ the firm's liquidity can cover operating costs in the growth phase with probability one, and if $W \geq \Lambda_{2}+I$ the firm's liquidity can cover the investment cost and operating costs in the mature phase with probability one. Naturally, for the firm to be permanently unconstrained in the growth phase it must be able to pursue both firstbest investment and liquidation strategies with probability one, so that the firm is financially unconstrained and attains the first-best value when $W \geq \max \left\{\Lambda_{1}, \Lambda_{2}+I\right\}$.

2. Medium Cash-holding Region: $I \leq W<\max \left\{\Lambda_{1}, \Lambda_{2}+I\right\}$. Consider next the situation where the firm has moderate financial slack. That is, when it has sufficient internal funds $W$ to cover the investment cost $I$ if it chooses to invest immediately, but not quite enough cash to ensure that it will never involuntarily liquidate its operating asset. The firm may not choose to exercise the growth option even when $Y$ reaches the first-best threshold $Y_{i}^{*}$, and even if it has sufficient internal funds $W$ to cover the investment cost $I$. The reason is that exercising the investment option would drain the firm of its cash to such an extent that the firm may be pushed to raise external funds prematurely to cover operating losses in the mature phase. The firm is therefore still financially constrained, even if it can entirely pay for the investment 
$I$ using internal funds, as liquidity concerns distort its investment and abandonment decisions.

At the endogenously chosen investment threshold, denoted by $\bar{Y}(W)$, firm value $G(W, Y)$ is continuous, so that $\bar{Y}(W)$ is an implicit function defined by the following value-matching condition:

$$
G(W, \bar{Y}(W))=P(W-I, \bar{Y}(W)) .
$$

In the medium cash-holding region the investment cost $I$ is entirely financed out of internal funds, so that post-investment liquidity $W$ decreases by $I$, as seen on the right side of (42).

3. Low Cash-holding Region: $0 \leq W<I$. The firm is even more severely constrained in this region as internal funds are insufficient to cover the investment cost $I$. The firm then has to raise external funds should it decide to invest. No matter how large the realization of its current $Y$ is, the firm has to access costly external capital markets if it chooses to invest immediately, as the investment cost $I$ is lumpy, $W<I$, while $Y$ is a flow variable. The post-financing/investment liquidity is then given by $W+F_{1}-I$. Again, at the moment of investing, the firm's value is continuous, so that the investment boundary $\bar{Y}(W)$ is implicitly defined by the following value-matching condition:

$$
G(W, \bar{Y}(W))=P\left(W+F_{1}-I, \bar{Y}(W)\right)-F_{1}-\Phi\left(F_{1}\right) .
$$

The right-hand side of condition (43) gives the firm's value in the mature phase after it has incurred investment cost $I$ and issued gross external funds $F_{1}+\Phi\left(F_{1}\right)$. The left-hand side of (43) is the firm's value before investing.

A firm without any cash $(W=0)$ can be in one of four possible situations:

a. When $Y \geq \bar{Y}(0)$, the firm raises external financing and exercises its growth option.

b. When $Z_{1} / m_{1} \leq Y<\bar{Y}(0)$, the firm is able to generate enough cash from operations to cover its operating cost, so that it needs no external financing.

c. When $\underline{Y}_{1}(0)<Y<Z_{1} / m_{1}$, the firm's internally generated cash-flow cannot fully cover its operating cost, and it is optimal to raise external funds so as 
to continue operations. We will characterize the liquidation threshold for a cash-less firm, $\underline{Y}_{1}(0)$, later on.

d. When $Y \leq \underline{Y}_{1}(0)$ it is best for the firm to liquidate its operating asset.

When the firm is indifferent between abandoning or continuing, the abandonment hurdle denoted by $\underline{Y}_{1}(W)$ is given by:

$$
G\left(W, \underline{Y}_{1}(W)\right)=W
$$

Next, we establish several key properties of the value function $G(W, Y)$.

Proposition 3 Under Assumptions 1-3 and 5, the following properties for $(W, Y) \in$ $\Sigma_{1}$ hold:

i) The solution for $G(W, Y)$ exists and is bounded as $W \leq G(W, Y) \leq G^{*}(W, Y)$.

ii) The solution for $G(W, Y)$ is strictly increasing in $W$ and strictly increasing in $Y$ before liquidation. Additionally, $H(W, Y)=G(W, Y)-W$ is strictly increasing in $W$.

iii) The solution for $G(W, Y)$ is unique.

Finally, we establish the following properties for the liquidation, external financing, and investment decisions.

Proposition 4 Under Assumptions 1-3, the following properties hold:

i) the optimal liquidation boundary is decreasing in $W$;

ii) the firm delays costly equity issuance until it has entirely exhausted its cash;

iii) if $P_{W}(0, \bar{Y}(I)) \geq \phi_{1}$ the investment threshold approaches infinity, i.e., $\bar{Y}(W) \rightarrow$ $\infty$, as $W \rightarrow I$ from the left.

The proofs for the technical results in this subsection are provided in Appendix C.

\section{Main Results}

We begin by motivating our choice of parameter values. We then proceed to numerically solve the model and provide economic intuition for the firm's optimal liquidation, external financing, and investment policies. Our numerical solution is 
based on the variational inequality method in the preceding section. To ease our exposition, we also use the more familiar real-options formulation that relies on valuematching and smooth-pasting conditions to characterize liquidation and investment decisions, but note that the equity-issuance decision for the survival purpose does not satisfy the smooth-pasting condition. ${ }^{11}$

\subsection{Parameter Values}

Investors are risk neutral as is standard in the real options and dynamic corporate finance literatures. However, for calibration purposes, we recognize that investors are risk averse. Using the standard asset pricing methodology, we can interpret our preceding analysis on the risk-adjusted basis by treating all our analysis as being done under the risk-neutral measure. Next, we explicitly incorporate the firm's risk premium and beta. ${ }^{12}$

Let $\mu^{P}$ denote the drift of the process $Y$ under the physical measure, $\rho$ the correlation coefficient between the shocks to the firm's fundamentals $Y$ and the economywide systematic shock, and $\eta$ the price of risk for the aggregate stock market. The drift of $Y$ under the risk-neutral measure is then given by $\mu=\mu^{P}-\rho \eta \sigma$. The model described thus far can then be understood as one in which the firm's risk premium is fully incorporated through the change from the physical measure to the risk-neutral measure.

Following Leland (1994) and others papers in the literature, we set the annual risk-free interest rate at $r=0.05$ and the annual revenue growth volatility at $\sigma=0.1$. Hence, if we take an expected growth rate of $\mu^{P}=0.014$ under the physical measure, a correlation coefficient of $\rho=0.8$, and a market price of risk of $\eta=0.3$, we obtain an expected growth rate of $\mu=\mu^{P}-\rho \eta \sigma=0.014-0.8 \times 0.3 \times 0.1=-0.01$ under the risk-neutral measure.

For the growth phase, we normalize the annual operating cost and the revenue multiple to $Z_{1}=1$ and $m_{1}=1$, respectively. Furthermore, to simplify the interpretation of our results we assume that the firm's growth option delivers an identical additional operating asset, so that earnings are doubled from $m_{1} Y-Z_{1}=Y-1$ to

\footnotetext{
${ }^{11}$ In our model, these two approaches yield the same solution. Appendix D provides a sketch of the solution algorithm.

${ }^{12}$ See Duffie (2001) for details on the equivalent martingale measure (risk-neutral) measure and how to connect the physical measure and the risk-neutral measure via Girsanov's Theorem.
} 
$m_{2} Y-Z_{2}=2(Y-1)$ when the firm exercises its growth option. We also normalize the investment cost to $I=1$.

For the external financing costs, we choose a low and a high level for the fixed cost $\phi_{0}$ for illustrative purposes: $\phi_{1}=0.01$ and $\phi_{0}=0.5$. For brevity, we focus on the case with $\phi_{1}$. Finally, we take the marginal financing cost to be $\phi_{1}=0.01$.

First-Best. The first-best liquidation hurdle in the growth phase is $Y_{a, 1}^{*}=0.767$. This implies that an unconstrained firm will continue as a going concern even when it incurs a loss as high as $Z_{1}-m_{1} Y_{a, 1}^{*}=0.333$, or $33.3 \%$ of the operating cost $Z_{1}=1$. The first-best liquidation hurdle in the mature phase is slightly higher, $Y_{a, 2}^{*}=0.8$. This means that the firm is less willing to absorb losses in the mature phase, as it is ready to abandon operations as soon as it incurs a loss larger than $Z_{2}-m_{2} Y_{a, 2}^{*}=0.4$, or $20 \%$ of operating costs $Z_{2}=2$.

The firm exercises its growth option when $Y$ reaches the investment hurdle $Y_{i}^{*}=$ 1.3. At that level, the project value (including the value of the abandonment option) is $Q^{*}\left(Y_{i}^{*}\right)=8.39$, and the NPV is $H^{*}\left(Y_{i}^{*}\right)=Q^{*}\left(Y_{i}^{*}\right)-I=7.39$. The minimum $W$ needed for a firm to be permanently financially unconstrained in the mature phase is

$$
\Lambda_{2}=\frac{r-\gamma \mu}{r^{2}(1-\gamma)} Z_{2}=8,
$$

which is four times the operating cost $Z_{2}=2$. In the growth phase this lower bound is $\Lambda_{1}=4.66$, so that the firm is permanently financially unconstrained if $W \geq \max \left\{\Lambda_{1}, I+\Lambda_{2}\right\}=9$.

\subsection{Inefficient Liquidation or External Financing for Sur- vival?}

Financial constraints both distort investment and impose external financing costs on the firm. As a result, a firm is prone to abandon operations sooner than the first-best timing.

\subsubsection{Liquidation Policy}

Liquidation in the growth phase involves the additional opportunity cost of losing any future growth options. Therefore, the firm is less willing to liquidate operations in the growth phase than in the mature phase, ceteris paribus. 

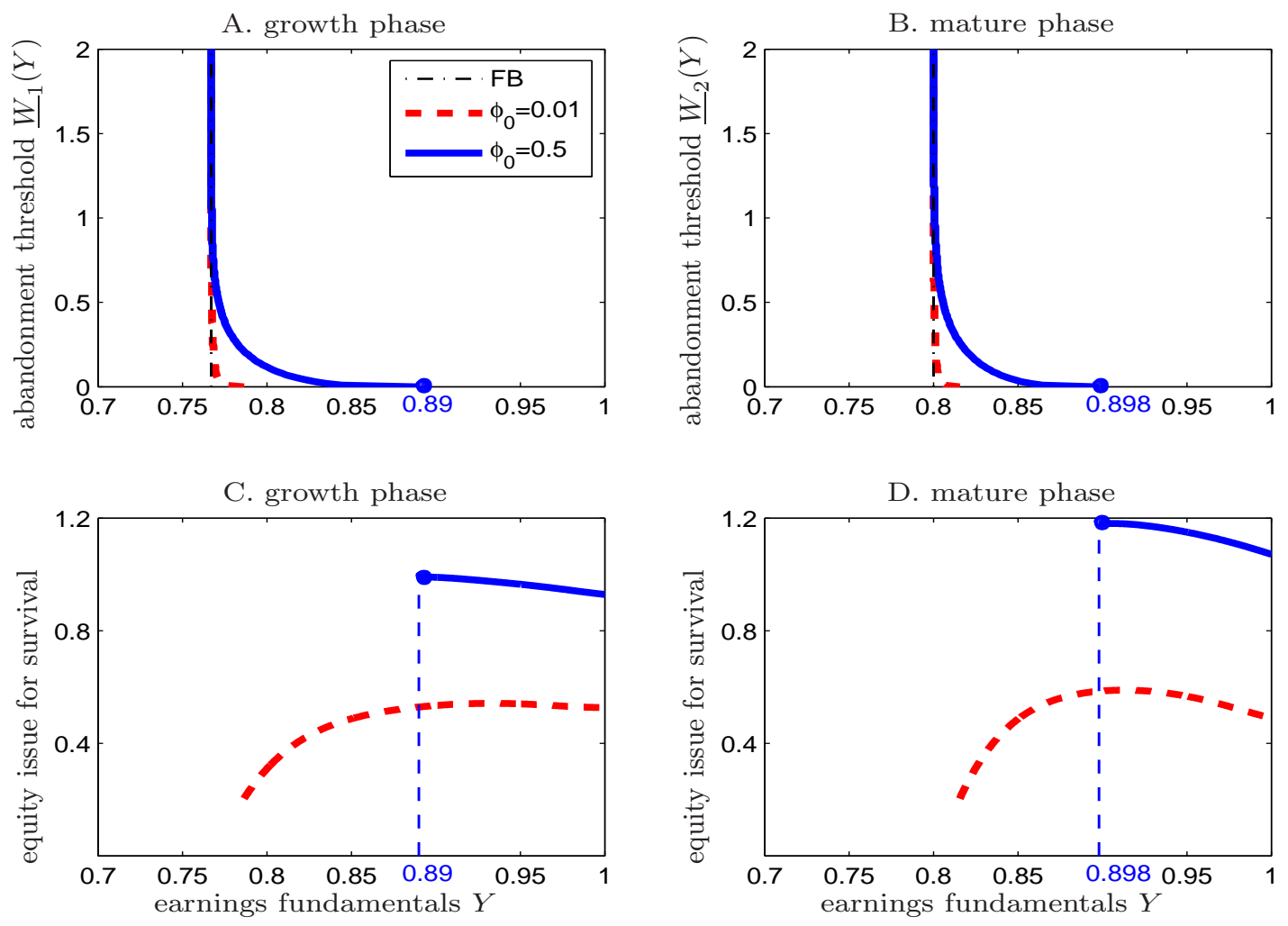

Figure 1: Abandonment hurdle, $\underline{W}_{1}(Y)$ and $\underline{W}_{2}(Y)$, and equity issuance for survival, $F_{1}(Y)$ and $F_{2}(Y)$. Panels $\mathrm{A}$ and $\mathrm{B}$ plot the abandonment hurdles in the growth and mature phases, respectively. Panels $\mathrm{C}$ and $\mathrm{D}$ plot the equity issuance in the growth and mature phases, respectively.

The Growth Phase. Panel A of Figure 1 plots the optimal liquidation hurdle $\underline{W}_{1}(Y)$ in the growth phase. The first-best abandonment threshold $Y_{a, 1}^{*}=0.767$ is indicated by the dotted line labeled MM. When the firm faces an external financing cost of $\phi_{0}=0.5$, it abandons operations sooner than the first-best, i.e., whenever $Y \leq 0.89$. Note also that the firm may abandon operations even before it runs out of cash. That is, $\underline{W}_{1}(Y)>0$ for $Y<0.89$. For example, when $Y=0.8$, the abandonment threshold for cash holdings is $\underline{W}_{1}(0.8)=0.12$. Finally, the lower the earnings, the higher the firm's $W$ when it abandons operations.

Moreover, as Panel A shows, $\underline{W}_{1}(Y)$ is a strictly decreasing function, indicating that the firm is increasingly eager to abandon as $Y$ approaches $Y_{a, 1}^{*}$. In other words, the higher are the firm's cash holdings $W$ the less inefficient is the firm's liquidation 
decision. The impact of external financing costs on the firm's liquidation policy essentially disappears when the firm's liquidity holding $W$ reaches 1.2 . Note finally that when the firm faces almost no fixed external financing cost, $\phi_{0}=0.01$, its abandonment policy closely approximates the first-best policy.

The Mature Phase. Panel B of Figure 1 plots the liquidation hurdle $\underline{W}_{2}(Y)$ for the mature phase. The shape of $\underline{W}_{2}(Y)$ is similar to the liquidation frontier in the growth phase. The main difference with the liquidation policy in the growth phase is that the firm is strictly more conservative in its continuation decisions in the mature phase. Compared with the first-best liquidation hurdle, $Y_{a, 2}^{*}=0.8$, a firm facing external financing costs $\phi_{0}=0.5$, liquidates sooner, i.e., when $Y$ reaches the level of 0.898. The reason is that the firm has no growth option in the mature phase and therefore has a lower continuation value than in the growth phase, other things equal (by construction we have constant returns to scale, $m_{1} / Z_{1}=m_{2} / Z_{2}=1$ ).

\subsubsection{Equity Issuance: $F_{1}(Y)$ and $F_{2}(Y)$}

In an environment with time-invariant external financing costs, as in our model, the firm is always better off delaying costly equity issuance as long as it can, as it is then able to save the time value of money on these financing costs. With a strictly positive liquidity buffer $W$ the firm can always cover any flow operating losses, so that it never issues equity before it entirely exhausts its cash.

In sum, the firm will only decide to raise costly external equity if it runs out of cash, or if it wants to invest and has insufficient internal funds to cover the fixed investment cost $I$. In the growth phase the firm chooses to issue equity at $W=0$, therefore,

$$
G(0, Y)=G^{F}(0, Y)
$$

in the region where $\underline{Y}_{1}(0)<Y<Z_{1} / m_{1}$. Otherwise, when $Y \leq \underline{Y}_{1}(0)$, the firm is liquidated and hence $G(0, Y)=0$.

By revealed preferences, the dividing boundary $\underline{Y}_{1}(0)$ is higher than the firstbest abandonment hurdle $Y_{a, 1}^{*}$. Should the firm seek to raise new funds, its optimal external financing amount $F_{1}$ is given by the FOC: ${ }^{13}$

$$
G_{W}\left(F_{1}, Y\right)=1+\Phi^{\prime}\left(F_{1}\right)=1+\phi_{1}, \quad \underline{Y}_{1}(0)<Y<Z_{1} / m_{1} .
$$

\footnotetext{
${ }^{13}$ We verify the second-order condition (SOC) to ensure that the FOC solution yields the maximal value.
} 
That is, the marginal value of cash $G_{W}\left(F_{1}, Y\right)$ must equal the marginal cost of external financing $1+\Phi^{\prime}\left(F_{1}\right)$. Note that the firm's marginal value of cash $G_{W}(W, Y)$ depends on its earnings fundamentals $Y$, and $G_{W}(W, Y)$ is greater than 1 at the moment of financing.

Similarly, in the mature phase, the firm only considers issuing equity at $W=0$. Conditional on issuing equity, the firm's value is

$$
P(0, Y)=P^{F}(0, Y)
$$

in the region where $\underline{Y}_{2}(0)<Y<Z_{2} / m_{2}$. Otherwise, when $Y \leq \underline{Y}_{2}(0)$, the firm is liquidated and hence $P(0, Y)=0$. Should the firm issue equity, the optimal external financing amount $F_{2}$ satisfies the following FOC:

$$
P_{W}\left(F_{2}, Y\right)=1+\Phi^{\prime}\left(F_{2}\right)=1+\phi_{1} .
$$

Panels C and D of Figure 1 plot the amount raised in the growth and mature phases, $F_{1}(Y)$ and $F_{2}(Y)$, when the firm has exhausted all internal funds and prefers to continue rather than abandon operations. When external financing is costly $\left(\phi_{0}=\right.$ 0.5) the firm prefers to continue if $Y \geq 0.89$ in the growth phase and $Y \geq 0.898$ in the mature phase. When funding is cheap $\left(\phi_{0}=0.01\right)$ the firm prefers to continue as long as $Y \geq 0.78$ in the growth phase and $Y \geq 0.81$ in the mature phase.

The two plots for $F_{n}(Y)$ can be understood as follows: the lower is $Y$, the more funds are needed to cover operating losses $Z_{n}-m_{n} Y$; at the same time, when $Y$ is lower the value of continuation is also lower. The tension between these two considerations generally translates into a non-monotonic function $F_{n}(Y)$. For low values of $Y, F_{n}(Y)$ is increasing in $Y$, and for higher values of $Y, F_{n}(Y)$ is decreasing in $Y$. This can clearly be seen for the (dotted) plots of $F_{n}(Y)$ when $\phi_{0}=0.01 .{ }^{14}$ Finally, note that when $m_{n} Y>Z_{n}$ the firm is able to accumulate cash from retained earnings and does not need any external funds.

In sum, the firm's expectation about its future profitability influences its current financing policy. This is an important difference with Bolton, Chen, and Wang (2011), where the firm's expected profitability is constant over time.

In addition, the firm raises more if the fixed costs of external funding $\phi_{0}$ are higher. This can be seen for example, at $Y=0.9$ : when the fixed issuance cost $\phi_{0}$ increases

\footnotetext{
${ }^{14}$ It is not apparent for the plot of $F_{n}(Y)$ when $\phi_{0}=0.5$, as the fixed cost is then so large that the firm prefers to abandon rather than continue with unprofitable operations and limited funds.
} 
from 0.01 to 0.5 , the firm's external financing, $F_{1}(0.89)$, then increases from 0.534 to 0.99. A firm that faces a larger fixed issuance cost wants to raise more funds to avoid going back to the capital markets. This prediction differs from those based on static models such as Froot, Scharfstein, and Stein (1993) and Kaplan and Zingales (1997), where the higher the fixed financing cost is the more financially constrained the firm is, and consequently the lower is the amount of equity financing it demands (the firm has no future financing considerations by assumption).

Next, we turn to the firm's investment decisions.

\subsection{The Growth Option: Internal versus External Financing}

Static models, Froot, Scharfstein, and Stein (1993) and Kaplan and Zingales (1997), also predict that firms with more internal funds invest more, as their overall funding costs are lower. Again, this prediction does not extend to a dynamic model in which firms can optimally time their investment. The main result of this section is that firms can become increasingly conservative in exercising their growth option as they accumulate internal funds. The reason is that by delaying investment they may be able to avoid having to turn to costly external funds altogether. The closer firms are to entirely funding their investment outlays $I$ with internal funds $W$, the higher the marginal expected return to delaying investment until they have entirely closed the funding gap $I-W$.

The Investment Timing Decision. Panels A and B of Figure 2 plot the investment hurdle $\bar{Y}(W)$ for two values of the fixed external financing cost, $\phi_{0}=0.01$ and $\phi_{0}=0.5$ in the regions $0 \leq W<I=1$ and $W \geq 1$ respectively. Recall that a firm is financially unconstrained in the growth phase if its liquidity $W$ is greater than $I+\Lambda_{2}=9$. We plot $\bar{Y}(W)$ for $W$ only up to $W=2.4$ in Panel B because the investment threshold effectively converges to the first-best hurdle $Y_{i}^{*}=1.30$ as $W \rightarrow 2.4$. Consider first the region where the firm's internal funds $W$ are sufficient to cover its investment cost $I=1$ (Panel $B$ ). In this region the firm finances its investment outlays entirely out of internal funds. As the plot of $\bar{Y}(W)$ shows, the higher is $W$ the less distorted is the firm's investment timing decision. With a higher $W$, the firm's mature value is higher, as it is less likely to run out of cash. As a result, the firm is eager to invest sooner.

Consider next the low cash-holding region $W \in[0, I)$. Panel A of Figure 2 plots 

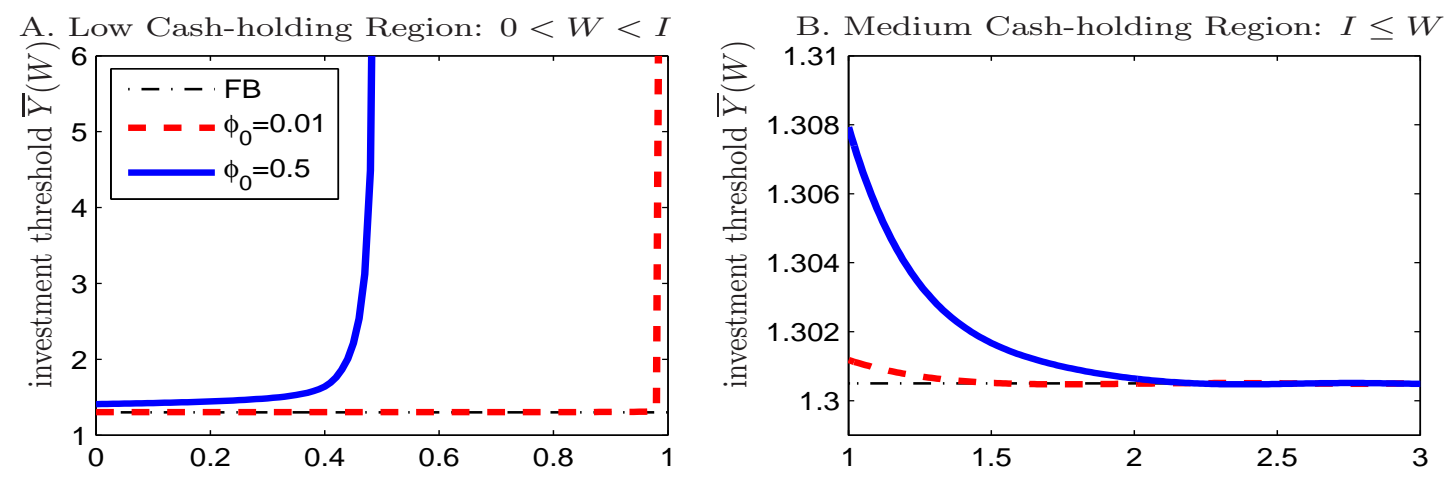

C. Low Cash-holding Region: $0<W<I$

D. Medium Cash-holding Region: $I \leq W$
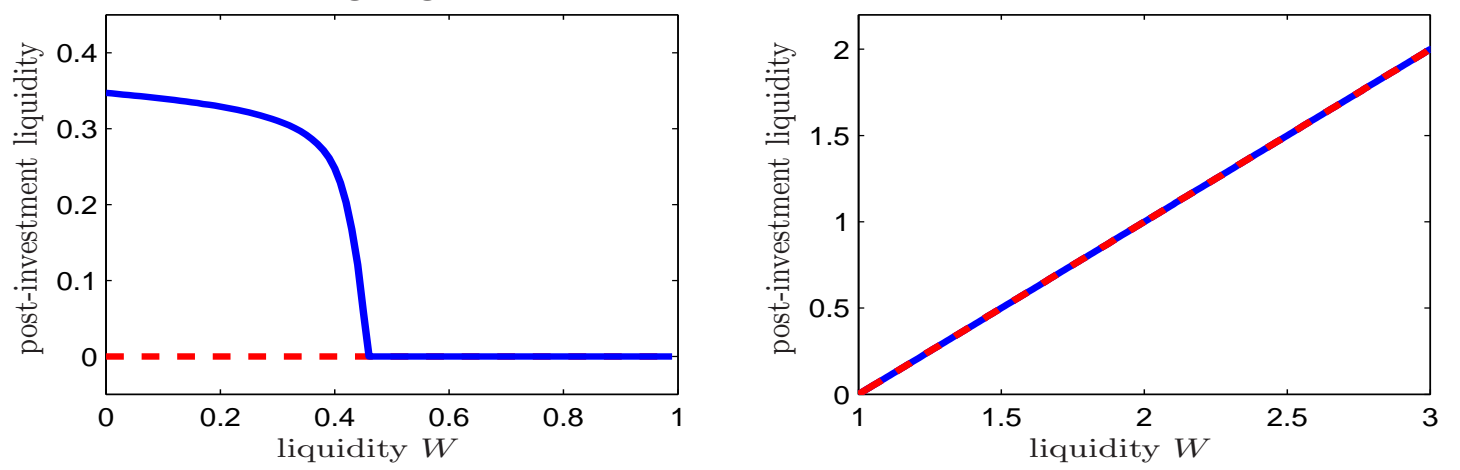

Figure 2: Investment hurdle $\bar{Y}(W)$ and the post-investment liquidity level $W+F_{1}(\bar{Y}(W))-I$. Panels A and B plot the investment hurdle $\bar{Y}(W)$ in the growth phase. Panels $\mathrm{C}$ and $\mathrm{D}$ plot the post-investment liquidity $W+F_{1}(\bar{Y}(W))-I$ at the transition into the mature phase.

the investment hurdle $\bar{Y}(W)$ as a function of $W$ in the region $[0,1)$. When $\phi_{0}=0.5$, $\bar{Y}(W)$ is strictly increasing and convex in $W$, going from 1.409 at $W=0$ to $+\infty$ as $W$ tends $I=1$. When $\phi_{0}=0.01, \bar{Y}(W)$ is weakly increasing and convex in $W$.

This remarkable result is exactly the opposite from the prediction of a static model. Far from behaving like a less constrained firm as $W \rightarrow I$, it behaves like a much more constrained firm. What is the logic behind this behavior? In essence, as $W \rightarrow I$ the firm increasingly wants to avoid the fixed external financing cost by waiting until it has accumulated sufficient internal funds rather than taps costly external funds to finance its investment as the firm is very close to accumulate enough funds to cover investment costs. Therefore, it therefore takes a larger and larger expected earnings fundamentals $Y$ to motivate the firm to invest immediately via costly external financing. 
Another way of understanding this result is that the firm's investment-timing decision trades off the value of the option to delay against the present cost of financing the investment. When the firm is financially constrained both the valuations of the timing option and the cost of investment vary with the marginal value of cash. In other words, for a firm the valuation of the timing option and the investment cost are fundamentally interconnected through the marginal value of cash, unlike in the standard textbook real options analysis.

The financing decision upon investment. Panels $\mathrm{C}$ and $\mathrm{D}$ of Figure 2 plot the firm's post-investment liquidity at time $\tau_{i+}, W_{\tau_{i}+}=W_{\tau_{i}}+F_{1}\left(W_{\tau_{i}}\right)-I$, where $F_{1}\left(W_{\tau_{i}}\right)$ denotes the net amount of external funds raised when the firm decides to invest at time $\tau_{i}$. The following conditions hold under optimal external financing: either

$$
P_{W}\left(W+F_{1}(W)-I, \bar{Y}(W)\right)=1+\phi_{1}, \text { and } F_{1}(W) \geq I-W,
$$

or

$$
P_{W}\left(W+F_{1}(W)-I, \bar{Y}(W)\right) \leq 1+\phi_{1} \text {, and } F_{1}(W)=I-W .
$$

Condition (50) states that the marginal value of cash at investment equals the marginal cost of financing. In this case, the firm issues at least $I-W$. Condition (51) is when the optimum is at the corner. In this case, the firm's net equity issue is $I-W$ and together with internal funds $W$, the firm's total funds is just sufficient to cover its investment cost $I .{ }^{15}$

When $W<I$, the firm is less willing to invest as $W$ increases. In other words, the firm's investment hurdle $\bar{Y}(W)$ increases with $W$, so that post investment the firm is better able to generate internal cash flows from operations when pre-investment liquidity $W$ is higher. Therefore, the amount of post-investment liquidity $W_{\tau_{i}+}$ needed decreases with the firm's current liquidity $W$, as shown in Panel C. When the firm faces low external financing costs $\left(\phi_{0}=0.01\right)$ the post-investment liquidity is essentially zero at all levels of $W$, since the firm only needs to obtain liquidity to cover the financing gap $I-W$. Panel D also illustrates that $F_{1}\left(W_{\tau_{i}}\right)$, the optimal amount of external funds raised when the firm decides to invest, is equal to 0 when $W \geq I$,

\footnotetext{
${ }^{15}$ In this case, the firm's marginal value of liquidity at investment must be weakly lower than the marginal cost of issuance. If this were not true, the firm would have chosen to issue equity in excess of $I-W$, which in turn equates the marginal value of cash with the marginal cost of equity issue. But this is the first case that we have already discussed.
} 
so that $W_{\tau_{i}+}=W_{\tau_{i}}-I$. This result is simply a consequence of the dynamic pecking order of financing principle.

\subsection{Value Loss due to Costly External Financing}

Our next set of results establishes how large the loss in firm value due to external financing costs can be. This loss stems directly from the financing costs per se and indirectly from the distortions in investment and abandonment policies. A key conclusion from our quantitative analysis is that low operating earnings fundamentals $Y$ and low cash buffers $W$ compound in making the loss particularly large, as Figure 3 illustrates.

This result at the individual firm's level for a stand-alone firm contributes to the downward-spiral adverse-feedback mechanism in the financial intermediation and crisis literature. For example, Brunnermeier and Sannikov (2014) show how an adverse shock to the fundamentals (capital shock in their model) weakens a financially constrained productive sector's balance sheet, which further causes underinvestment generating an adverse feedback loop from the fundamentals shock to the equilibrium price of capital.

Panels A and C plot the firm's enterprise value $H(W, Y)=G(W, Y)-W$ in the growth phase for two values of $Y$, a low value $Y=0.82$ for which the firm incurs operating losses, and a high value, $Y=1.18$, for which the firm makes a profit.

Enterprise value $H(W, Y)$ is, of course, lower the higher are external financing costs $\phi_{0}$. As the plots for $H(W, Y)$ illustrate, the loss is particularly large when $\phi_{0}=0.5, Y=0.82$, and the firm runs out of cash. When $W$ approaches 0 the entire first-best enterprise value is then essentially wiped out. In contrast, when $\phi_{0}=0.01$ the firm loses only around $10 \%$ of the first best value when $W$ approaches 0 . And when $Y=1.18$ the loss in value is small even when financing costs are high.

A simple yet robust insight from these results is that the significance of external financing costs varies considerably with the firm's operating revenues. One should therefore not expect to see a stable relation between firm value and external financing costs in the data.

Panels B and D of Figure 3 plot the firm's enterprise value in the mature phase:

$$
Q(W, Y)=P(W, Y)-W
$$

The same qualitative results on value loss obtain in the mature phase as in the growth 

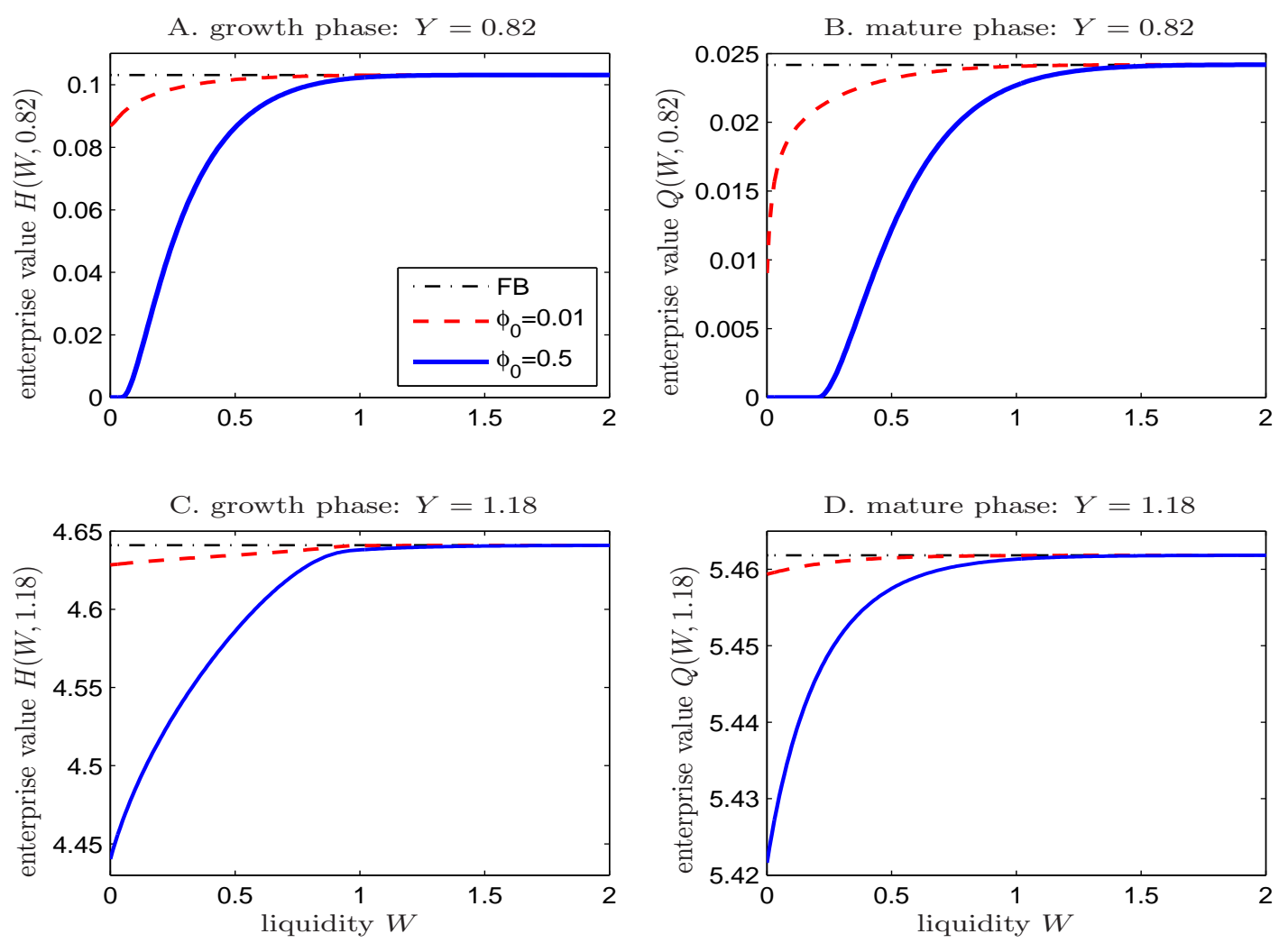

Figure 3: Enterprise values in the growth and mature phases, $H(W, Y)$ and $Q(W, Y)$. Panels A and B plot $H(W, Y)$ and $Q(W, Y)$ for $Y=0.82$. Panels $\mathrm{C}$ and $\mathrm{D}$ plot $H(W, Y)$ and $Q(W, Y)$ for $Y=1.18$.

phase, although the size of the losses is somewhat smaller.

In sum, these results illustrate how operating earnings fundamentals $Y$ and internal funds $W$ are substitutes in terms of mitigating financial constraints. Moreover, abstracting from financial flexibility considerations when determining the firm's real option decisions-as most of the real options literature has done-could generate highly misleading predictions and vastly overstate the value of real options. Equally, an exclusive focus on financial frictions that ignores the firm's real flexibility-as is the case for most of the corporate financial constraints literature-risks understating the value of precautionary savings. 


\begin{tabular}{|c|c|c|c|c|c|}
\hline \multirow[t]{2}{*}{$\begin{array}{c}\text { time } \\
t\end{array}$} & $\begin{array}{c}\text { firm's } \\
\text { earning } Y_{t}\end{array}$ & $\begin{array}{l}\text { net equity } \\
\text { issue } F_{t}\end{array}$ & $\begin{array}{c}\text { gross equity } \\
\text { issue } F_{t}+\Phi\left(F_{t}\right)\end{array}$ & $\begin{array}{c}\% \text { of equity issue } \\
\omega_{t+}\end{array}$ & $\begin{array}{l}\text { original investors' } \\
\text { ownership } \alpha_{t+}(\%)\end{array}$ \\
\hline & \multicolumn{5}{|c|}{ Panel A: $W_{0}=0.1$} \\
\hline 0 & 1 & 0 & 0 & 0 & $100 \%$ \\
\hline 1.16 & 0.913 & 0.98 & 1.49 & $30.1 \%$ & $69.9 \%$ \\
\hline 2.04 & 1.314 & 0 & 0 & 0 & $69.9 \%$ \\
\hline 10.4 & 0.931 & 1.17 & 1.68 & $82.6 \%$ & $12.2 \%$ \\
\hline \multirow[t]{2}{*}{23.8} & 0.890 & 0 & 0 & 0 & liquidation \\
\hline & \multicolumn{5}{|c|}{ Panel B: $W_{0}=0.2$} \\
\hline 0 & 1 & 0 & 0 & 0 & $100 \%$ \\
\hline 2.23 & 1.443 & 1.11 & 1.62 & $12.8 \%$ & $87.2 \%$ \\
\hline 12.9 & 0.921 & 1.18 & 1.69 & $88.3 \%$ & $10.2 \%$ \\
\hline 24.4 & 0.926 & 1.17 & 1.68 & $85.1 \%$ & $1.5 \%$ \\
\hline 37.6 & 0.839 & 0 & 0 & 0 & liquidation \\
\hline
\end{tabular}

Table 1: Equity ownership dynamics and (investment, equity issue, and abandonment) timing decisions for a simulated path. The parameter values are $r=0.05, \mu=-0.01, \sigma=0.1, I=1 m_{1}=Z_{1}=1, m_{2}=Z_{2}=2, \phi_{1}=0.01$ and $\phi_{0}=0.5$. For the given simulated path with $Y_{0}=1$, we consider two cases with $W_{0}=0.1$ and $W_{0}=0.2$. Under the first-best case, the investment time is $t=2.03$ and the abandonment time is $t=39.5$.

\section{Simulating the Firm's Life-Cycle}

To provide further insight into the dynamics of investment, SEOs, cash management, and abandonment decisions, we simulate one sample path of earnings fundamentals $Y$ with two initial cash holding values, $W_{0}=0.1$ and $W_{0}=0.2$, with baseline parameter values and $\phi_{0}=0.5$. The evolution of corporate decisions are illustrated in Figures 4 and 5, respectively. Table 1 reports the decisions and calculates the implied equity ownership dynamics that follow from the firm's decisions over the simulated path used in Figures 4 and 5.

In the MM world, the optimal investment time for this sample path is $t=2.03$, just when the firm's earnings $Y_{t}$ reach 1.3, and the abandonment time is $t=39.5$, just as the firm's earnings $Y_{t}$ hit the low level of 0.8. Thus, the firm is willing to fund losses of up to $20 \%$ of operating costs $Z_{2}=2$ under this scenario to maximize the value of its abandonment option.

Recall that when the firm incurs an external financing cost $\Phi\left(F_{t}\right)$ as it raises 
equity $F_{t}$ at time $t$, the firm's post-issuance value is $P\left(W_{t+}, Y_{t+}\right)$. Let $\omega_{t+}$ denote the equilibrium fraction of the newly issued equity held by outside investors. By competitive market pricing, we have:

$$
\omega_{t+}=\frac{F_{t}+\Phi\left(F_{t}\right)}{P\left(W_{t+}, Y_{t+}\right)}=1-\frac{P\left(W_{t}, Y_{t}\right)}{P\left(W_{t+}, Y_{t+}\right)},
$$

as the new investors just break even under perfectly competitive capital markets. Under the simulated sample-path we can highlight the dynamics of equity dilution by keeping track of the equity ownership of the original investors who have stayed with the firm since its inception. We denote by $\alpha_{t}$ the ownership share of the original equity holders at time $t$, with $\alpha_{0}=1$ by construction. As the firm issues equity to finance investment and/or replenish liquidity over time, the original equity investors' ownership then evolves as follows:

$$
\alpha_{t+}=\alpha_{t}\left(1-\omega_{t+}\right)
$$

In other words, with no issuance $\omega_{t+}=0$ and $\alpha_{t+}=\alpha_{t}$, so that $\alpha$ does not change. But when new equity is issued at time $t$, with a strictly positive ownership stake for new investors of $\omega_{t+}>0$, the original equity investors' equity is diluted to $\alpha_{t+}$ from $\alpha_{t}$ according to (54).

Figure 4 plots the scenario where the firm starts with a low cash stock of $W_{0}=0.1$. Panel A plots the path of earnings fundamentals $Y_{t}$ starting with $Y_{0}=1$. Note first that when the firm faces external financing costs at $t=1.16$ where it exhausts its liquidity as $W_{1.16}=0$ and $Y_{1.16}=0.913$, and the firm issues net equity of $F_{1}(0.913)=$ 0.98 to replenish its liquidity from $W_{1.16}=0$ to $W_{1.16+}=0.98$, by selling $\omega_{1.16+}=$ $30.1 \%$ of its equity, and the equity share is $\alpha_{1.16}=69.9 \%$.

And then it exercises its investment option at time $t=2.04$ when $Y_{t}$ reaches 1.314 . It then pays the investment cost $I=1$ solely out of internal funds, thus depleting its stock of cash $W_{2.04+}$ down to 0.057, as illustrated in Panel B. In this case, obviously there is no equity dilution and $\alpha_{2.04}=69.9 \%$.

Next, at $t=10.4$, when the firm's fundamentals $Y_{10.4}$ falls to 0.931 and it has exhausted its savings, the firm issues net equity of $F_{2}(0.931)=1.17$ to replenish its liquidity from $W_{10.4}=0$ to $W_{10.4+}=1.17$, by selling $\omega_{10.4+}=82.6 \%$ of its equity. At that point the firm's original owners are nearly wiped out and only retain a stake of $\alpha_{10.4+}=12.2 \%$. 
A. a sample path $Y$ with $Y_{0}=1$

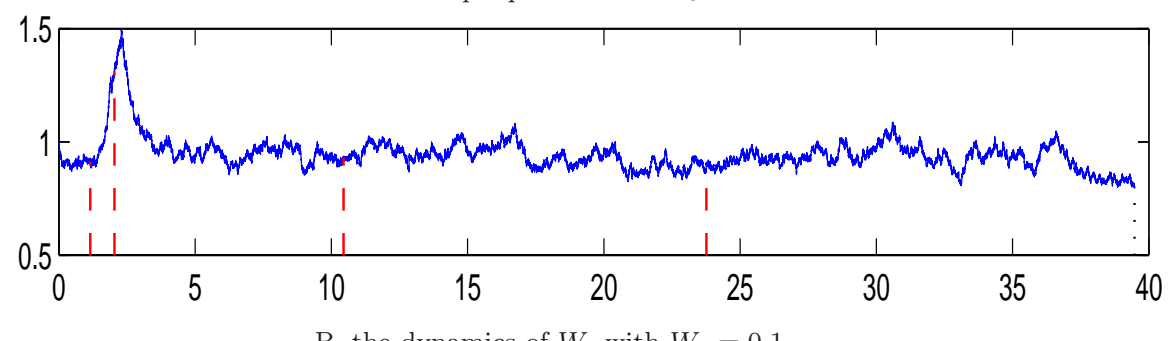

B. the dynamics of $W_{t}$ with $W_{0}=0.1$
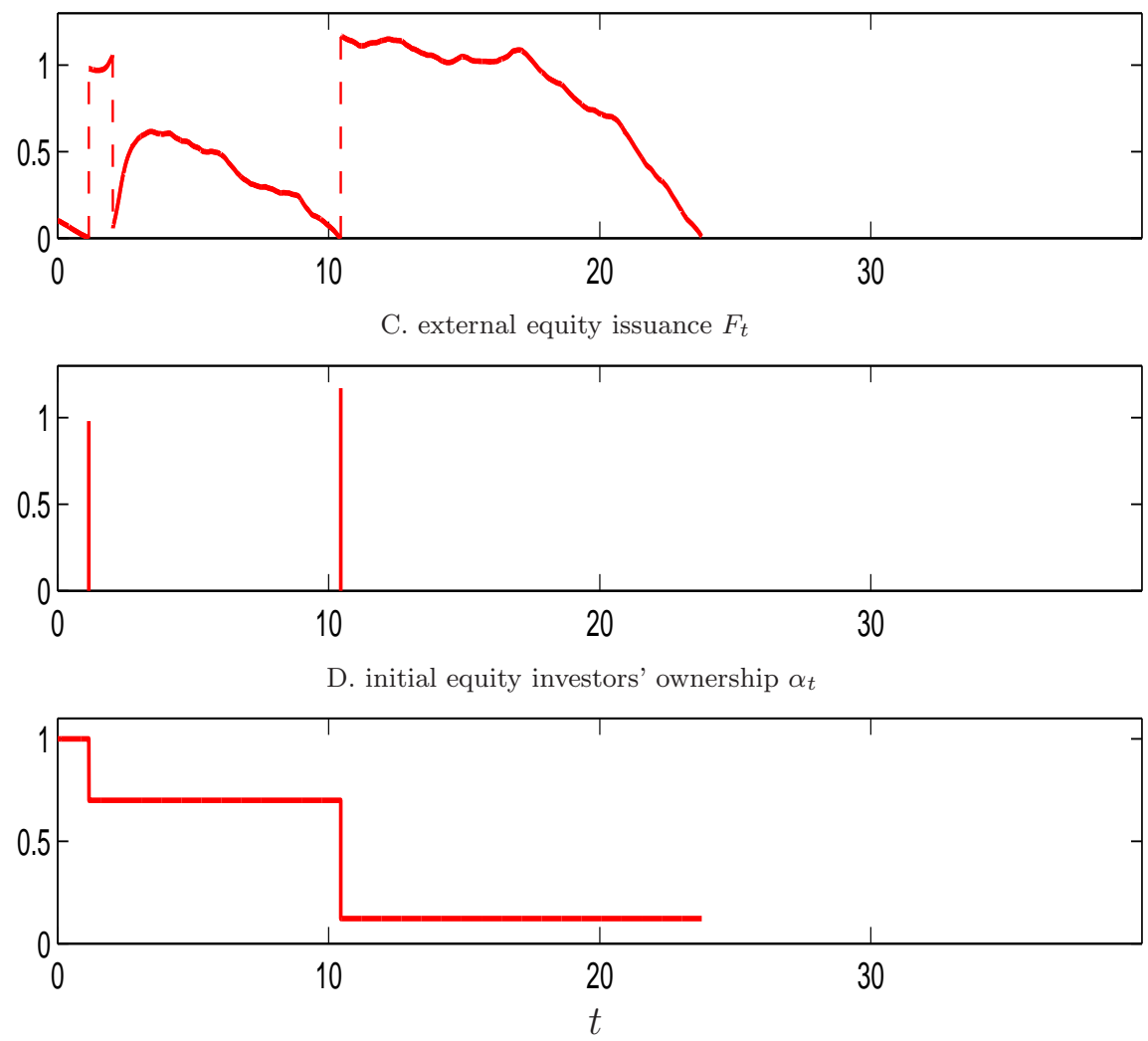

Figure 4: Investment, liquidity accumulation, external equity issue, ownership dynamics, and liquidation for a simulated path. The firm's initial cash holding is $W_{0}=0.1$.

Finally, at $t=23.8$, when the firm runs out cash for the second time and $Y_{t}$ reaches 0.890 , it simply abandons its asset. At this low point the cost of new external financing simply outweighs the benefit of keeping the firm as a going concern.

Figure 5 plots the scenario where the firm starts with a higher cash stock of $W_{0}=0.2$. Panel A again plots the identical simulated path of earnings fundamentals 
A. a sample path $Y$ with $Y_{0}=1$

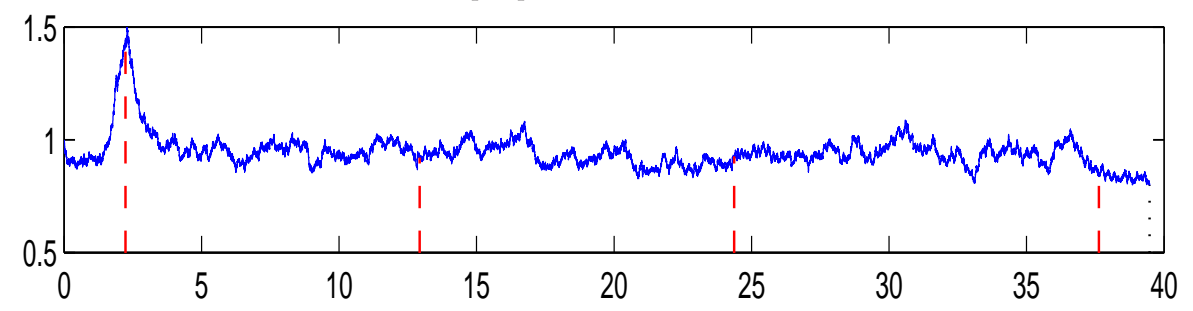

B. the dynamics of $W_{t}$ with $W_{0}=0.2$

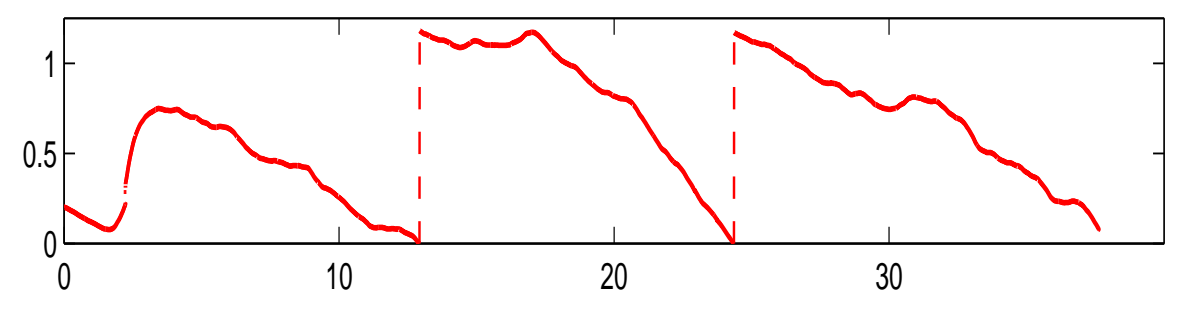

C. external equity issuance $F_{t}$

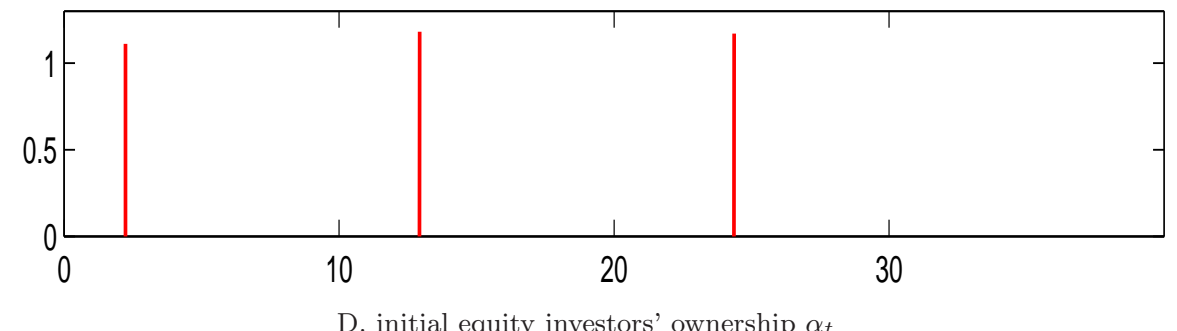

D. initial equity investors' ownership $\alpha_{t}$

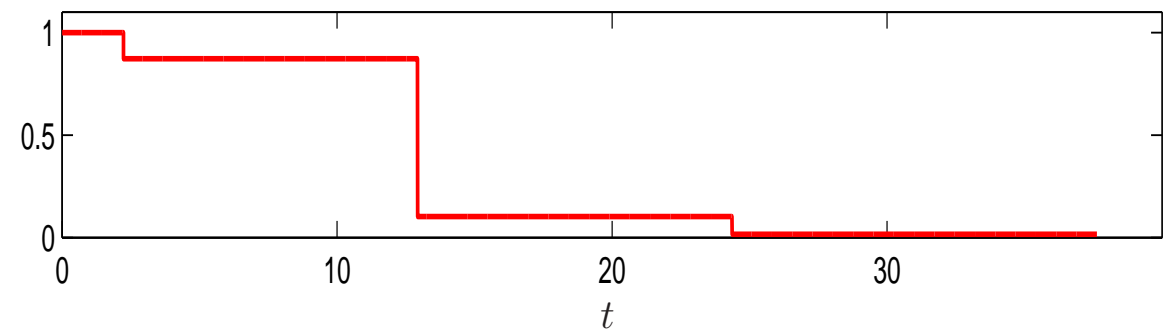

Figure 5: Investment, liquidity accumulation, external equity issue, ownership dynamics, and liquidation for a simulated path. The firm's initial cash holding is $W_{0}=0.2$.

$Y_{t}$ starting with $Y_{0}=1$. As the firm's $Y_{t}$ reaches 1.443 at $t=2.23$, the firm finances its investment cost $I=1$ via a combination of external equity $(F(1.443)=1.11)$ by issuing a fraction of $\omega_{2.23+}=12.8 \%$ firm's equity and internal funds (0.22), leaving the firm with a stock of post-investment cash of $W_{2.23+}=0.33$ (as shown in Panel B). As a result, the original owners are diluted down to an ownership stake of $\alpha_{2.23+}=87.2 \%$ 
(as shown in Panel D).

At $t=12.9$, when earning $Y_{t}$ has collapsed to 0.921 and the firm's liquidity has been drained, the firm returns to the capital markets and raises a net amount of $F_{2}(0.921)=1.18$ by selling $\omega_{12.9+}=88.3 \%$ of the firm's equity, thus further diluting the firm's original owners' down to $\alpha_{12.9+}=10.2 \%$ (see Panel D). Next, at $t=24.4$, as the firm's liquidity $W_{t}$ is again drained out, the firm yet again issues equity by selling $\omega_{18.6+}=85.1 \%$ of the firm's equity, raising a total net amount of $F=1.17$ and diluting the original owners down to a small stake of $\alpha_{24.4+}=1.5 \%$. Finally, at $t=37.6$ the firm almost runs out of cash again and abandons its asset given that expected operating earnings hit the low level of $Y_{t}=0.839$; it then distributes the remaining cash 0.08 to its shareholders.

Comparing the two scenarios, we make the following observations: First, in all cases where the firm issues equity for purposes of replenishing its liquidity it chooses different financing levels because each time it faces different expected operating earnings when it exhausts its liquidity. Second, the firm with $W_{0}=0.1$ turns out to abandon its asset sooner (at $t=23.8$ ) than the firm with $W_{0}=0.2$ (at $t=37.6$ ), which runs counter to the conventional wisdom that a firm with more initial liquidity abandons its operations later.

\section{Cash-carry Costs and Payout Policy}

A helpful simplification in our analysis so far has been that there is no opportunity cost for the firm in holding cash. In reality, however, firms do face an opportunity cost in holding cash and therefore do decide to pay out some of their accumulated profits when retained earnings are high. Accordingly, in this section we extend the model by introducing cash-carry costs. We now assume that instead of earning the market interest rate $r$ on its cash savings the firm only earns $(r-\lambda)$, where $0<\lambda<r$ represents the firm's unit cash-carry cost.

In this case, it is sometimes optimal for the firm to pay out before it closes operations. Specifically, there is an endogenous upper boundary $\bar{W}(Y)$ such that it is strictly optimal for the firm to pay out any excess cash $W-\bar{W}(Y)$ to its shareholders. With cash-carry cost $\lambda$, the cash accumulation process is as follows, in the growth phase

$$
d W_{t}=\left((r-\lambda) W_{t}+m_{1} Y_{t}-Z_{1}\right) d t+d C_{t}-d U_{t}, \quad W_{t} \geq 0
$$


and in the mature phase

$$
d W_{t}=\left((r-\lambda) W_{t}+m_{2} Y_{t}-Z_{2}\right) d t+d C_{t}-d U_{t}, \quad W_{t} \geq 0
$$

When $Y<\infty$, there exists a payout boundary $0<\bar{W}(Y)<\infty$ such that the value of an additional retained dollar is exactly equal to the value of paying out that dollar to shareholders. And if the firm starts out with $W>\bar{W}(Y)$, it distributes the difference $W-\bar{W}(Y)$ as a lump sum and thereby brings down the cash stock to $\bar{W}(Y)$. Since firm value must be continuous before and after cash distribution, the value $P(W, Y)$ for $W>\bar{W}(Y)$ is given by

$$
P(W, Y)=P(\bar{W}(Y), Y)+(W-\bar{W}(Y)), \quad W>\bar{W}(Y)
$$

All other optimality conditions remain the same as in the baseline model.

The firm's payout decision is jointly determined with its investment and liquidity management policies. In the growth phase, the firm has no reason to make any payout if its earnings fundamentals $Y$ are relatively high but still lower than the endogenous investment boundary, because it then expects to invest and expand its operating capacity in the future. ${ }^{16}$

As Fama and French (2001) have noted, growth firms tend to delay dividend payments as they expect to spend their retained earnings on investment outlays. However, when operating earnings fundamentals $Y$ are so low that expansion is unlikely, the firm may prefer to make a payout to shareholders rather than inefficiently burn through its cash holdings. Thus, in the growth phase one would expect the firm to make payouts when $Y$ is low and not when $Y$ is high. In contrast, in the mature phase, one would expect the firm to be more eager to pay out retained earnings the higher are its operating revenues.

The results shown in Figures 6 and 7 confirm these insights. These two figures display the firm's optimal decisions-the payout, inaction, investment and liquidation regions-for any pair $(W, Y)$. The parameter values are the same as for the baseline model, with the addition of the cash carry cost $\lambda=0.02$.

There are three regions in the mature phase, each with a single optimal action. When $(W, Y)$ are to the right of the dashed red payout boundary and when $Y$ exceeds

\footnotetext{
${ }^{16}$ If its earnings fundamentals $Y$ is even higher than the firm's optimal investment boundary, the firm optimally immediately invests, becoming a mature firm. Our analysis for the mature firm applies accordingly.
} 
the dotted black line, the firm optimally pays out some of its earnings, as shown in the payout region of Figure 6 . When $(W, Y)$ take intermediate values in the "inaction region" bordered by the (dashed red) payout boundary, the (solid blue) liquidation boundary, and the $y$-axis, it is optimal for the firm to continue operations while retaining all of its earnings. When $Y$ is below the solid blue line, which extends into the dotted black liquidation-line, the firm is in the "liquidation region". It is then best for the firm to abandon operations.

The dashed, red payout-frontier is downward sloping over most of the region $0.9<$ $Y<1.35$, illustrating the basic tradeoff the firm faces: when the internal cash-flow generating machine is less productive it is worth building a larger retained earnings buffer.

Note, however, that there is a small non-monotonic segment of the payout boundary. This segment is explained by the fact that when $Y$ is less than $Z_{2} / m_{2}=1$, the firm is making operating losses so that there are two competing forces at play: 1) building a larger liquidity buffer to be able to absorb these loses and ride them out, which causes a downward sloping liquidation boundary, and 2) avoiding throwing good money after bad, which encourages earlier liquidation to minimize operating losses. The latter force dominates when the firm's operating performance is close to the solid, blue liquidation-boundary, and the former dominates when operating losses are relatively low.

Optimal policies in the growth phase are more intricate, as Figure 7 illustrates. The firm faces more decisions in this phase, so that there are five policy regions in total in this phase.

1. When $(W, Y)$ are high, with $W \geq I=1$, the firm invests by entirely funding the fixed investment cost out of internal funds, as is indicated by the Investment Only region. ${ }^{17}$

2. When $Y$ takes intermediate values and $W$ is high the firm does not invest but pays out some of its earnings, as indicated by the Payout region. In this region

\footnotetext{
${ }^{17}$ Note that when $Y \geq 1.29$, the firm immediately exercises its growth option provided that $W \geq 1$. This is in contrast to the investment policy displayed in Panel B of Figure 2, when the firm has no cash carry costs. In that case the firm somewhat delays its investment as $W$ approaches 1 from above. The reason is that the firm prefers to continue building up its cash buffer before investing, so as to continue with a higher cash (working capital) buffer after incurring the investment cost $I=1$. When the firm faces cash carry costs this motive is essentially undone by its desire to save on these costs.
} 


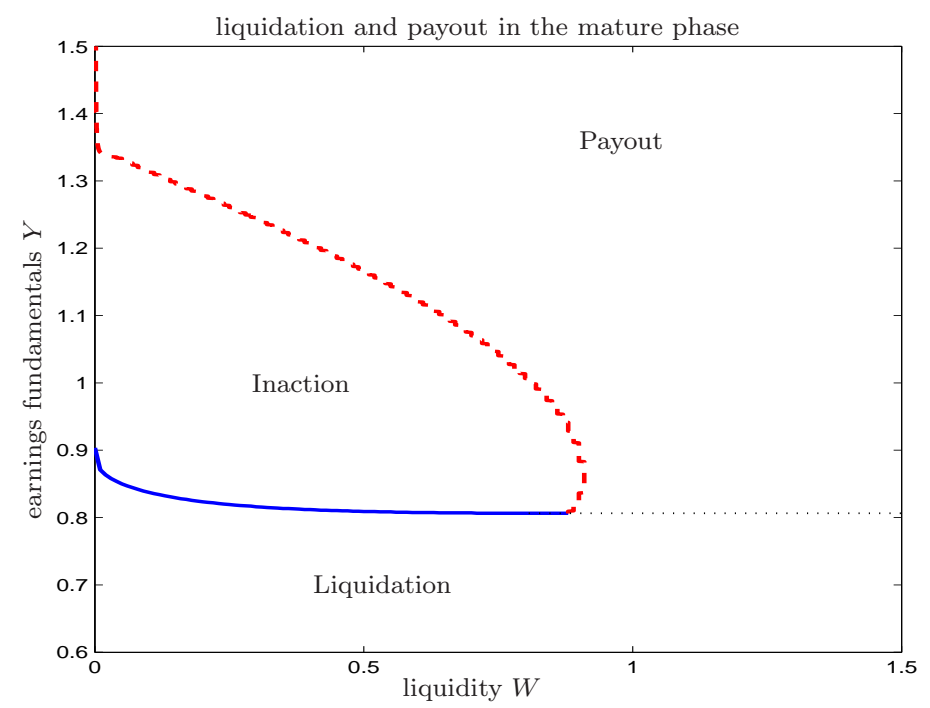

Figure 6: Liquidation and payout in the mature phase.

Parameters used: $r=0.05, \mu=-0.01, \sigma=0.1, m_{2}=Z_{2}=2, \phi_{1}=0.01, \phi_{0}=0.5$, $\lambda=0.02$. In the payout region, the firm optimally pays out a lump-sum dividend in order to reach the payout boundary. For example, a firm with $(W, Y)=(0.78,1.14)$ chooses to pay out a one-time dividend in the amount of 0.18 to reach $(0.6,1.14)$ on the boundary.

the operating asset is not sufficiently profitable to exercise the growth option. Given that the firm is not planning to immediately spend its internal funds on capital outlays, it prefers to pay out some of its liquidity buffer rather than pay the full cash carry cost $\lambda W$.

Note that the payout boundary $\bar{W}(Y)$ has a somewhat complex shape. This is again explained by the fact that when the firm is making losses $(Y<1)$ the two competing forces described above are at play. In addition, when $Y$ approaches the investment threshold $Y=1.29$ from below and when $W \geq 1$ the firm prefers to delay payout in anticipation of the future investment outlays and its greater need for internal funds.

3. For low values of $Y$ it is optimal for the firm to abandon operations and disburse any accumulated internal funds $W$, as is indicated by the Liquidation region.

4. For intermediate values of $(Y, W)$ the firm is optimally in a business as usual mode and does not take any significant decisions, whether it is investment, 


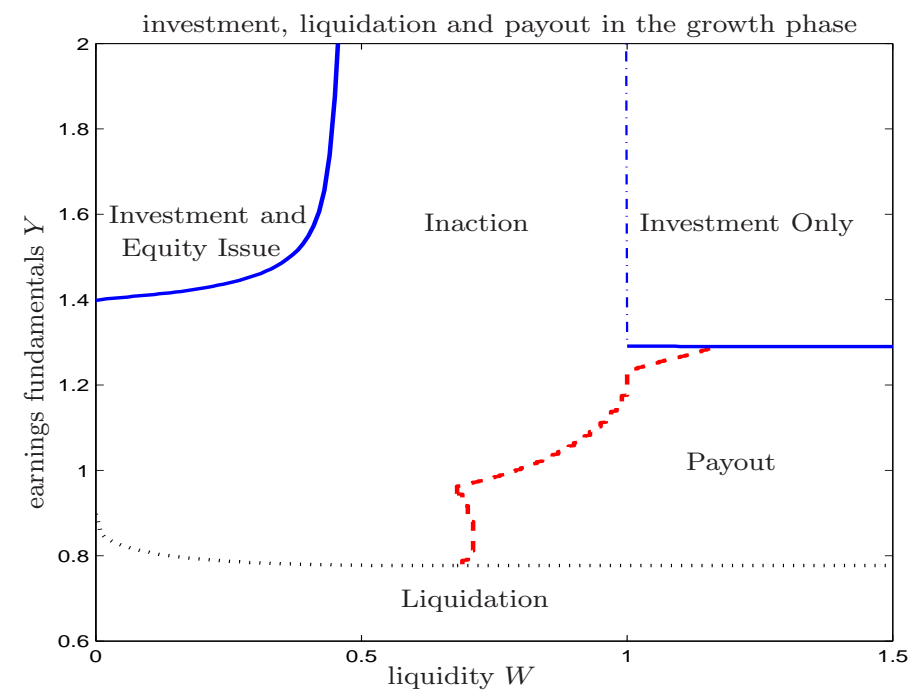

Figure 7: Investment, liquidation and payout in the growth phase.

Parameters used: $r=0.05, \mu=-0.01, \sigma=0.1, m_{1}=Z_{1}=1, m_{2}=Z_{2}=2$, $\phi_{1}=0.01, \phi_{0}=0.5, I=1, \lambda=0.02$.

abandonment, or external financing, as indicated by the Inaction region.

5. Finally, for values of $(Y, W)$ such that $Y$ is high but $W<1$, it is optimal for the firm to invest even if it requires external financing to cover the investment outlays, as is shown by the Investment and Equity Issue region. In this situation the firm uses a combination of funds from internal sources and from a (costly) external equity issue.

The effects of cash-carry costs on investment. Figure 8 illustrates the effects of cash-carry costs $\lambda$ on investment. Panel A exhibits the shift in the investment threshold induced by a change in $\lambda$ in the low-cash holding region $(W<I)$ where the firm has to raise some external equity to finance the investment cost $I$. Panel $\mathrm{B}$ plots the same relation in the medium-cash holding region $(W>I)$ where the firm has sufficient internal funds to finance its investment cost. In both panels, we find that the investment threshold is monotonically decreasing with the cash-carry cost $\lambda$. This is intuitive: the higher the cash-carry cost $\lambda$, the sooner the firm invests, as the opportunity cost of holding cash is higher. 

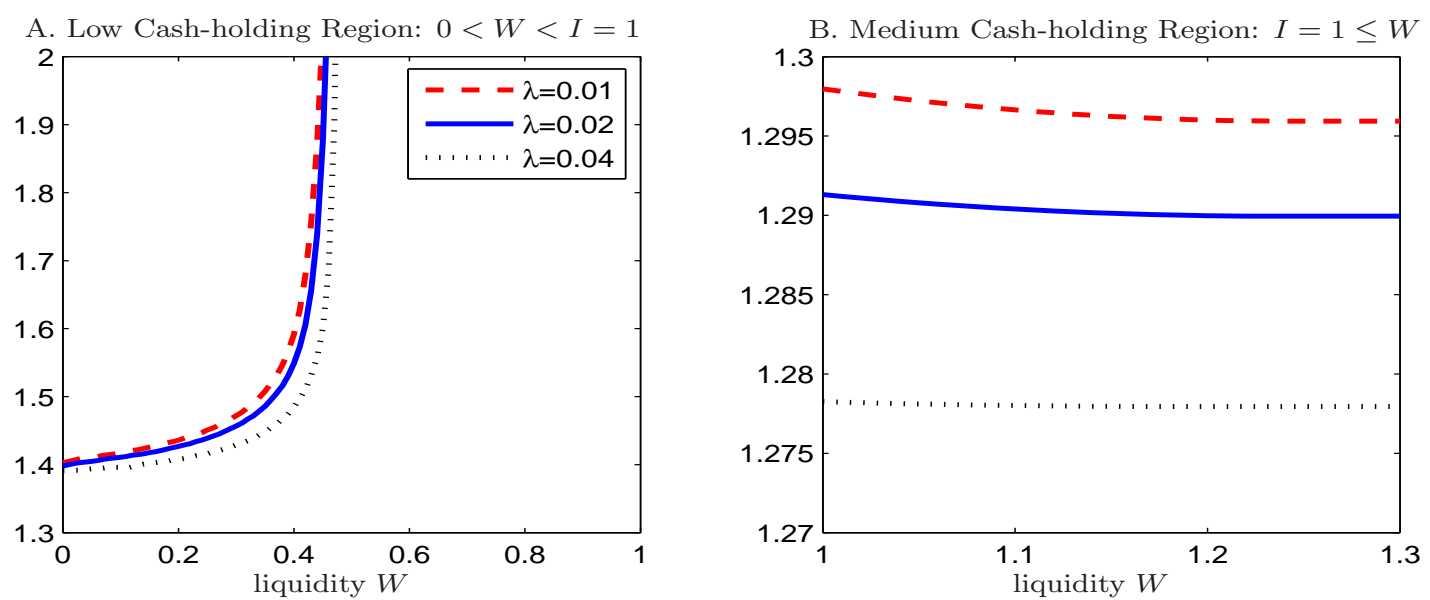

Figure 8: The effects of cash-carry cost $\lambda$ on the investment hurdle $\bar{Y}(W)$.

With internal financing of the investment (Panel B), the investment threshold also decreases with $W$ for a given $\lambda$, as there is a diminishing marginal value of holding cash when the cash buffer is larger. However, when external financing is needed (Panel A), the investment threshold for a given $\lambda$ is increasing in $W$, as the firm increasingly prefers to wait until it has accumulated sufficient internal funds to entirely cover the investment cost and thereby avoid costly external financing.

Overall, these results point to a simple and robust payout policy pattern: growth firms should only pay out retained earnings if their growth opportunities are unappealing; in contrast, mature firms should pay out retained earnings when their operating earnings are high, and should not when their earnings are low.

\section{Sequential Investment Opportunities}

To capture how future investment opportunities may influence a firm's current investment and financing decisions, we next generalize our model to allow for two rounds of investment options. We refer to stage 0 , when the firm has not yet exercised any investment option, as the start-up phase; stage 1, after the firm has made its first investment, as the growth phase; and, stage 2, after the firm has completed both investment rounds, as the mature phase.

This extension brings out one striking new effect: an acceleration of the first investment with the objective of building internal cash-flow generation capacity to 
relax financial constraints for the second round of investment. That is, we show that a firm may invest sooner than an otherwise unconstrained firm, in an effort to increase its capacity to generate cash.

The main change to the baseline model is the addition of a start-up phase in which the firm has no operating asset. In this phase the firm has two growth options. We denote the fixed cost of the $n$th growth option by $I_{n}$, with $n \in\{1,2\}$. As before, the revenue process generated by the operating assets is given by $m_{n} Y_{t}$ and the operating cost by $Z_{n}$. Once the firm has exercised its first growth option the model is identical to the baseline model. The mature and growth phases therefore inherit the same firm value, the same dynamics for liquidity, and the same abandonment decisions as in the baseline model.

In stage 0 , the start-up phase, the firm's liquidity $W_{t}$ accumulates as follows:

$$
d W_{t}=r W_{t} d t+d C_{t}, \quad W_{t} \geq 0
$$

where $\left\{C_{t} ; t \geq 0\right\}$ denotes as before the firm's cumulative equity issues, and $d C$ is the net equity raised. We denote by $S(W, Y)$ the value of the firm in this start-up phase. As in the growth and mature phases, $S(W, Y)$ satisfies the following conditions:

1. $S(W, Y) \geq W$,

2. $S(W, Y) \geq S^{F}(W, Y)=\sup _{F_{0} \geq 0} S\left(W+F_{0}, Y\right)-F_{0}-\Phi\left(F_{0}\right)$,

3. $S(W, Y) \geq S^{I}(W, Y)=\sup _{F_{0} \geq 0} G\left(W+F_{0}-I_{1}, Y\right)-F_{0}-\Phi\left(F_{0}\right)$.

In the interior operating region $S(W, Y)$ satisfies $\mathcal{L}_{0} S=0$, where $\mathcal{L}_{0}$ is the infinitesimal generator in the start-up phase:

$$
\mathcal{L}_{0} S=r W S_{W}+\mu Y S_{Y}+\frac{\sigma^{2} Y^{2} S_{Y Y}}{2}-r S
$$

Given that the analysis of the start-up phase proceeds along similar lines as the analysis of the baseline model, we only report the most striking novel result on the investment threshold policy $\bar{Y}_{1}(W)$ in the start-up phase (phase 0) when the firm has no asset in place at all. ${ }^{18}$

\footnotetext{
${ }^{18}$ In this generalized model, we have two rounds of investment decisions. Therefore, we use $\bar{Y}_{1}(W)$ and $\bar{Y}_{2}(W)$ to denote the first and second growth-option exercise-thresholds, respectively. Similarly, we use $Y_{i, 1}^{*}$ and $Y_{i, 2}^{*}$ to denote the first-best exercise-thresholds for the first and second growth-option.
} 


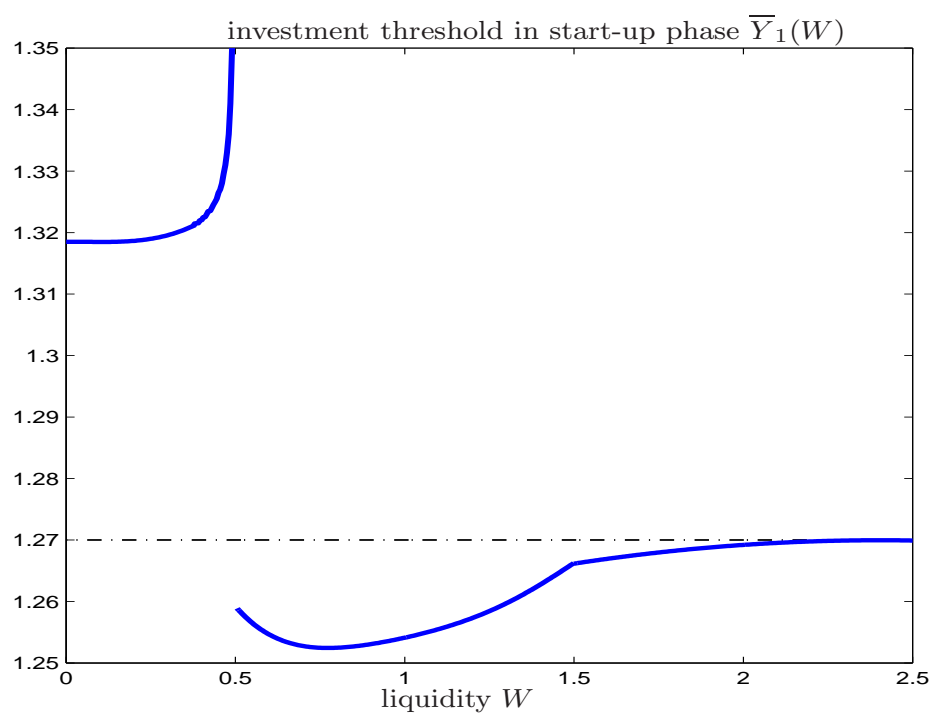

Figure 9: The optimal investment threshold $\bar{Y}_{1}(W)$ in the startup phase: The case with sequential growth options. Parameters used: $r=0.05, \mu=$ -0.01, $\sigma=0.1, m_{1}=Z_{1}=1, m_{2}=Z_{2}=2, \phi_{0}=0.5, \phi_{1}=0.01, I_{1}=0.5, I_{2}=1$.

We set $I_{1}=0.5$, at half the fixed investment cost for exercising of the second growth option. All the other parameter values are the same as for the solution in our baseline model. With these parameter values, the first-best investment threshold in the start-up phase is given by $Y_{i, 1}^{*}=1.27$.

Figure 9 plots the optimal investment threshold $\bar{Y}_{1}(W)$ for the first investment option over the interval $W \in[0,2.5]$. It shows that $\bar{Y}_{1}(W)$ is monotonically increasing in $W$ in the region $W<I_{1}=0.5$, consistent with our earlier findings for the baseline model.

The striking new result is for $W \geq 0.5$, when the firm invests more aggressively than under the first best: $\bar{Y}_{1}(W)<Y_{i, 1}^{*}=1.27$. The firm accelerates the timing of its first growth option in order to access the internal funds generated by the operating asset. By over-investing in the first growth option, the firm gains overall because the benefit of relaxing the financial constraint for the second growth option outweighs the cost of accelerating the first growth option.

This result reflects a more general principle: other things equal, financially constrained firms prefer growth opportunities with front-loaded cash-flows. In the classical MM-based real options framework there is a basic equivalence between an option 
that pays the present discounted value as a lump sum and an option that pays a cash flow of equivalent present value over time. This is not the case in our real-options model for a financially constrained firm as the over-investment result above illustrates. Our more general model can thus explain why in reality firms tend to prefer projects with front-loaded cash flows.

\section{Conclusion}

Our generalization of the classic real options framework of McDonald and Siegel (1986) to include financing considerations reveals the risks for corporations of overly relying on textbook real-options tools (that assume away financing costs) to guide their investment policies and to determine the value of their real options. Although the classical real option framework can provide a satisfactory approximation for firms with adequate internal funds and with highly profitable operating assets, we have shown that it is highly misleading for moderately profitable firms, with low or even medium levels of internal funds. For the latter firms the value of growth options is not only substantially lower than the estimates produced by classical option pricing tools, but also the lack of internal funds to finance capital expenditures results in significant deviations in the optimal investment policy from that prescribed by the classical real options model.

Our general real options framework with financial constraints is particularly relevant for entrepreneurial firms, for which growth options represent the most significant part of their value, and for which external financing costs are highest and internal funds tightest. The reason why these firms face high external financing costs is that they have little collateral to offer and therefore have to rely mostly on external equity financing, for which information dilution costs à la Myers and Majluf (1984) are very high. As our analysis of sequential investment options indicates, a strategy to relax financial constraints that some start-ups have adopted is to invest in an operating asset before the long-term viability of that asset could be ascertained, in an attempt to generate internal cash flows that could be useful towards financing future growth options.

Our analysis of payout policy also reveals how dividend and stock repurchase policies are tied to growth opportunities. In the presence of such opportunities it is

optimal for the firm not to pay out retained earnings, as internal funds are better 
deployed towards funding the firm's future capital expenditures. But once these opportunities have been seized, optimal payout policy flips: more profitable firms pay out more, exactly the opposite of the policy in the growth phase. The empirical literature on dividend policy (e.g. Fama and French, 2001) finds that growth firms do delay dividend payments and our analysis offers a simple explanation for this behavior.

Although the introduction of financial constraints in a real options model is a significant generalization, our model falls short of accurately representing several aspects of financial constraints that firms face in reality. For example, we have assumed for simplicity that external financing costs are the same whether the firm is in the start-up, growth, or mature phase. In reality, chances are that external financing costs are lower for firms with a proven track record or for firms who have assets in place that can serve as collateral. We have also suppressed any possibility of debt financing, including allowing the firm access to a line of credit it could draw down when it faces a liquidity squeeze. Accordingly, extending our model to allow for a richer and more realistic description of external financing options is an obvious next step in developing this framework further. 


\section{Appendices}

Appendix A provides some details for the First-Best solution. Appendix B and C provide proofs for the results for the general case with financial constraints in the mature phase and the growth phase, respectively. Finally, Appendix D provides a sketch of our numerical solution procedure.

\section{A Technical details for the First-Best solution}

Below we report the explicit solutions for the first-best abandonment hurdle in the mature phase $Y_{a, 2}^{*}$, the first-best investment threshold $Y_{i}^{*}$, and the first-best abandonment threshold $Y_{a, 1}^{*}$ in the growth phase. Sundaresan, Wang, and Yang (2015) provide derivational details for essentially the same first-best real-option problem. For brevity, we skip the derivations here.

The abandonment hurdle $Y_{a, 2}^{*}$ in the mature phase is given by :

$$
Y_{a, 2}^{*}=\frac{\gamma}{\gamma-1} \frac{r-\mu}{r} \frac{Z_{2}}{m_{2}}
$$

where the constant $\gamma$ is given by:

$$
\gamma=\frac{1}{\sigma^{2}}\left[-\left(\mu-\frac{\sigma^{2}}{2}\right)-\sqrt{\left(\mu-\frac{\sigma^{2}}{2}\right)^{2}+2 r \sigma^{2}}\right]<0 .
$$

The investment threshold $Y_{i}^{*}$ and abandonment threshold $Y_{a, 1}^{*}$ in the growth phase jointly solve the following equations:

$$
\begin{aligned}
0 & =\frac{m_{1}-m_{2}}{r-\mu}+\frac{\beta\left(Y_{a, 1}^{*}\right)^{\gamma}\left(Y_{i}^{*}\right)^{\beta-1}-\gamma\left(Y_{a, 1}^{*}\right)^{\beta}\left(Y_{i}^{*}\right)^{\gamma-1}}{\left(Y_{a, 1}^{*}\right)^{\gamma}\left(Y_{i}^{*}\right)^{\beta}-\left(Y_{a, 1}^{*}\right)^{\beta}\left(Y_{i}^{*}\right)^{\gamma}}\left(Q^{*}\left(Y_{i}^{*}\right)-I-\left(\frac{m_{1} Y_{i}^{*}}{r-\mu}-\frac{Z_{1}}{r}\right)\right) \\
& -\frac{(\gamma-\beta)\left(Y_{i}^{*}\right)^{\beta+\gamma-1}}{\left(Y_{a, 1}^{*}\right)^{\gamma}\left(Y_{i}^{*}\right)^{\beta}-\left(Y_{a, 1}^{*}\right)^{\beta}\left(Y_{i}^{*}\right)^{\gamma}}\left(\frac{m_{1} Y_{a, 1}^{*}}{r-\mu}-\frac{Z_{1}}{r}\right)-\gamma\left(\frac{Y_{i}^{*}}{Y_{a, 2}^{*}}\right)^{\gamma-1}\left(\frac{Z_{2}}{r}-\frac{m_{2} Y_{a, 2}^{*}}{r-\mu}\right),
\end{aligned}
$$

and

$$
\begin{aligned}
0= & \frac{m_{1}}{r-\mu}+\frac{(\beta-\gamma)\left(Y_{a, 1}^{*}\right)^{\gamma+\beta-1}}{\left(Y_{a, 1}^{*}\right)^{\gamma}\left(Y_{i}^{*}\right)^{\beta}-\left(Y_{a, 1}^{*}\right)^{\beta}\left(Y_{i}^{*}\right)^{\gamma}}\left(Q^{*}\left(Y_{a, 1}^{*}\right)-I-\left(\frac{m_{1} Y_{i}^{*}}{r-\mu}-\frac{Z_{1}}{r}\right)\right) \\
& -\frac{\gamma\left(Y_{i}^{*}\right)^{\beta}\left(Y_{a, 1}^{*}\right)^{\gamma-1}-\beta\left(Y_{i}^{*}\right)^{\gamma}\left(Y_{a, 1}^{*}\right)^{\beta-1}}{\left(Y_{a, 1}^{*}\right)^{\gamma}\left(Y_{i}^{*}\right)^{\beta}-\left(Y_{a, 1}^{*}\right)^{\beta}\left(Y_{i}^{*}\right)^{\gamma}}\left(\frac{m_{1} Y_{a, 1}^{*}}{r-\mu}-\frac{Z_{1}}{r}\right),
\end{aligned}
$$


where the constant $\gamma$ is given by (A.2) and the constant $\beta$ is given by:

$$
\beta=\frac{1}{\sigma^{2}}\left[-\left(\mu-\frac{\sigma^{2}}{2}\right)+\sqrt{\left(\mu-\frac{\sigma^{2}}{2}\right)^{2}+2 r \sigma^{2}}\right]>1 \text {. }
$$

\section{B Proofs for the general case in the mature phase}

\section{B.1 Proof for Lemma 1}

For any given time $t$, we can construct a control policy $\widehat{A} \in \mathcal{A}$ with $\widehat{A}=\left\{\left(\tau_{L}^{2}=\right.\right.$ $\left.\left.t, \tau_{F}^{2}>t, F_{2}\right)\right\}$ such that the firm is liquidated immediately at time $t$. Under this policy, we have

$$
P\left(W_{t}, Y_{t} ; \widehat{A}\right)=W_{t} .
$$

Since $P\left(W_{t}, Y_{t}\right)=\sup _{A \in \mathcal{A}} P\left(W_{t}, Y_{t} ; A\right) \geq P\left(W_{t}, Y_{t} ; \widehat{A}\right)$, it immediately follows that $P(W, Y) \geq W$.

\section{B.2 Proof for Lemma 2}

For any given time $t$, we can construct a control policy $\widehat{A} \in \mathcal{A}$ with $\widehat{A}=\left\{\left(\tau_{L}^{2}>\right.\right.$ $\left.\left.t, \tau_{F}^{2}=t, F_{2} \geq 0\right)\right\}$ such that the firm issues equity at time $t$ and for any $s \geq t$ we assume that the firm takes the optimal liquidation and financing decisions, including financing timing and the choice of the optimal amount $F_{2}$. Under the above policy, the (optimal) value function at time $t$ is $P\left(W_{t-}+F_{2}, Y_{t-}\right)-F_{2}-\Phi\left(F_{2}\right)$ where $t-$ is the left limit of $t$. By the continuity of value function upon the equity issue, we have

$$
P\left(W_{t-}, Y_{t-} ; \widehat{A}\right)=P\left(W_{t-}+F_{2}, Y_{t-}\right)-F_{2}-\Phi\left(F_{2}\right)
$$

Since the time of the equity issue for $\tau_{F}^{2}=t$ may not be optimal, we have

$$
\sup _{A \in \mathcal{A}} P\left(W_{t-}, Y_{t-} ; A\right) \geq \sup _{F_{2} \geq 0} P\left(W_{t-}, Y_{t-} ; \widehat{A}\right)=\sup _{F_{2} \geq 0} P\left(W_{t-}+F_{2}, Y_{t-}\right)-F_{2}-\Phi\left(F_{2}\right) .
$$

Recall that $P\left(W_{t-}, Y_{t-}\right)=\sup _{A \in \mathcal{A}} P\left(W_{t-}, Y_{t-} ; A\right)$, so that

$$
P\left(W_{t-}, Y_{t-}\right) \geq \sup _{F_{2} \geq 0} P\left(W_{t-}+F_{2}, Y_{t-}\right)-F_{2}-\Phi\left(F_{2}\right)
$$

\section{B.3 Proof for Lemma 3}

See details in the main text following Lemma 3. 


\section{B.4 Proof for Lemma 4}

First, for sufficiently high $W$, i.e. $W \geq \Lambda_{2}$, the firm is permanently financially unconstrained and hence Lemma 4 directly follows from Lemma 3.

Next, we consider the case when the firm is financially constrained, i.e., when $W_{t}<\Lambda_{2}$. By applying Lemma 1 and Lemma 2 to (13), we obtain

$$
P\left(W_{t}, Y_{t}\right) \geq \mathbb{E}_{t}\left[e^{-\left(\tau_{U}^{2}-t\right)} P^{*}\left(W_{\tau_{U}^{2}}, Y_{\tau_{U}^{2}}\right)\right] \geq \mathbb{E}_{t}\left[e^{-\left(\tau_{U}^{2}-t\right)} P\left(W_{t}, Y_{\tau_{U}^{2}}\right)\right]
$$

Here, the first inequality follows from the fact that the first two terms in (13) are positive and the second inequality follows from $W_{\tau_{U}^{2}}=\Lambda_{2}>W_{t}$ and the result that the value function is monotonic in $W$, as stated in Proposition 1.

Consider a small time interval $(t, t+\Delta t)$ where $\Delta t>0$. As $Y_{t} \rightarrow \infty$, the firm abandons its operations or issues equity with zero probability. This is because $W_{t+\Delta t}>\Lambda_{2}$, as $Y_{t} \rightarrow \infty$. One can check this result by using the dynamics of $W_{t}$, given in (5) to this case. That is, as $Y_{t} \rightarrow \infty, \tau_{U}^{2} \rightarrow t$ with probability one. When $\tau_{U}^{2} \rightarrow t,(\mathrm{~B} .1)$ boils down to

$$
P\left(W_{t}, Y_{t}\right) \geq P^{*}\left(W_{t}, Y_{t}\right) \geq P\left(W_{t}, Y_{t}\right)
$$

That is, $P\left(W_{t}, Y_{t}\right)=P^{*}\left(W_{t}, Y_{t}\right)$ if $\tau_{U}^{2} \rightarrow t$. Put differently, the firm achieves the first-best outcome and hence $P(W, Y)=P^{*}(W, Y)=W+Q^{*}(Y)$ when $Y \rightarrow \infty$.

\section{B.5 Proof for Theorem 2}

Verification. We establish that any piecewise $\mathcal{C}^{2}$ function which is a solution to equation (25) associated to problem (13) is a majorant of the value function $P$.

Proposition 5 (Verification) Suppose we can find a positive function $\widetilde{P}$ which is piecewise $\mathcal{C}^{2}$ on the region $\Sigma_{2}$ with bounded first derivatives ${ }^{19}$ and such that for all $(W, Y) \in \Sigma_{2}$

$$
\begin{aligned}
\mathcal{L}_{2} \widetilde{P} & \leq 0 \\
\widetilde{P}(W, Y) & \geq W \\
\widetilde{P}(W, Y) & \geq \sup _{F_{2} \geq 0} \widetilde{P}\left(W+F_{2}, Y\right)-F_{2}-\Phi\left(F_{2}\right)
\end{aligned}
$$

with boundary conditions $\widetilde{P}(W, Y)=P^{*}(W, Y)$ for $W=\Lambda_{2}$ or $Y \rightarrow \infty$, then $\widetilde{P}(W, Y)=P(W, Y)$ for all $(W, Y) \in \Sigma_{2}$.

\footnotetext{
${ }^{19}$ In the sense of Definition 4.8, p. 271 in Karatzas and Shreve (1988).
} 
Proof. We first show that $\widetilde{P}(W, Y) \geq P(W, Y)$. Notice that $A$ is a control policy in problem (13), where $A \in \mathcal{A}$ and $\mathcal{A}$ is the set of all policies. Then, by definition, we have (14). If we can show that $\widetilde{P}(W, Y) \geq P(W, Y ; A)$ for any control policy $A \in \mathcal{A}$, then it follows that $\widetilde{P}(W, Y) \geq P(W, Y)$.

Using the generalized Ito's formula (Dellacherie and Meyer (1980), Theorem VIII25 and Remark c, p. 349), we can write:

$$
\begin{gathered}
e^{-r\left(\tau_{2}-t\right)} \widetilde{P}\left(W_{\tau-}, Y_{\tau_{2}-}\right)=\widetilde{P}\left(W_{t}, Y_{t}\right)+\int_{t}^{\tau_{2}-} e^{-r(s-t)} \mathcal{L}_{2} \widetilde{P}\left(W_{s}, Y_{s}\right) d s \\
+\int_{t}^{\tau_{2}-} e^{-r(s-t)} \widetilde{P}_{Y}\left(W_{s}, Y_{s}\right) \sigma Y_{s} d B_{s}
\end{gathered}
$$

Since $\widetilde{P}$ satisfies (B.3), the second term of the right hand side is non-positive. Moreover, since the first derivative of $\widetilde{P}$ is bounded, the third term is a square integrable martingale. Taking expectations, we then get:

$$
\mathbb{E}_{t}\left[e^{-r\left(\tau_{2}-t\right)} \widetilde{P}\left(W_{\tau_{2}-}, Y_{\tau_{2}-}\right)\right] \leq \widetilde{P}\left(W_{t}, Y_{t}\right) .
$$

Suppose that the firm issues equity $N$ times from time 0 to time $\tau_{L}^{2} \wedge \tau_{U}^{2}$, and divide the time interval $\left(0, \tau_{L}^{2} \wedge \tau_{U}^{2}\right)$ into $N+1$ subperiods. We define $\tau_{F_{n}}^{2}$ as the time of the $n$-th round of external financing, where $1 \leq n \leq N$. During the last period $\left(\tau_{F_{N}}^{2}, \tau_{L}^{2} \wedge \tau_{U}^{2}\right)$ we then have:

$$
\begin{aligned}
\widetilde{P}\left(W_{t}, Y_{t}\right) & \geq \mathbb{E}_{t}\left[e^{-r\left(\tau_{2}-t\right)} \widetilde{P}\left(W_{\tau_{2}-}, Y_{\tau_{2}-}\right)\right] \\
& =\mathbb{E}_{t}\left[e^{-r\left(\tau_{2}-t\right)} \widetilde{P}\left(W_{\tau_{2}}, Y_{\tau_{2}}\right) \mathcal{I}_{\tau_{2}=\tau_{L}^{2}}+e^{-r\left(\tau_{2}-t\right)} P^{*}\left(W_{\tau_{2}}, Y_{\tau_{2}}\right) \mathcal{I}_{\tau_{2}=\tau_{U}^{2}}\right] \\
& \geq \mathbb{E}_{t}\left[e^{-r\left(\tau_{2}-t\right)} W_{\tau_{2}} \mathcal{I}_{\tau_{2}=\tau_{L}^{2}}+e^{-r\left(\tau_{2}-t\right)} P^{*}\left(W_{\tau_{2}}, Y_{\tau_{2}}\right) \mathcal{I}_{\tau_{2}=\tau_{U}^{2}}\right] \\
& =P\left(W_{t}, Y_{t} ; A\right)
\end{aligned}
$$

where we have used the condition (B.4) for the second inequality.

During the $N$-th external financing subperiod $\left(\tau_{F_{N-1}}^{2}, \tau_{2}\right)$ we have in turn:

$$
\begin{aligned}
\widetilde{P}\left(W_{t}, Y_{t}\right) & \geq \mathbb{E}_{t}\left[e^{-r\left(\tau_{2}-t\right)} \widetilde{P}\left(W_{\tau_{2}-}, Y_{\tau_{2}-}\right)\right] \\
& =\mathbb{E}_{t}\left[e^{-r\left(\tau_{2}-t\right)} \widetilde{P}\left(W_{\tau_{2}-}, Y_{\tau_{2}-}\right) \mathcal{I}_{\tau_{2}=\tau_{F_{N}}^{2}}\right] \\
& \geq \mathbb{E}_{t}\left[e^{-r\left(\tau_{2}-t\right)} \sup _{F_{2} \geq 0}\left[\widetilde{P}\left(W_{\tau_{2}-}+F_{2}, Y_{\tau_{2}-}\right)-F_{2}-\Phi\left(F_{2}\right)\right] \mathcal{I}_{\tau_{2}=\tau_{F_{N}}^{2}}\right] \\
& =\mathbb{E}_{t}\left[e^{-r\left(\tau_{2}-t\right)} \sup _{F_{2} \geq 0}\left[\mathbb{E}_{\tau_{F_{N}}^{2}}\left[\widetilde{P}\left(W_{\tau_{2}-}+F_{2}, Y_{\tau_{2}-}\right)\right]-F_{2}-\Phi\left(F_{2}\right)\right] \mathcal{I}_{\tau_{2}=\tau_{F_{N}}^{2}}\right] \\
& \geq \mathbb{E}_{t}\left[e^{-r\left(\tau_{2}-t\right)}\left[\mathbb{E}_{\tau_{F_{N}}^{2}}\left[P\left(W_{\tau_{2}-}+F_{2}, Y_{\tau_{2}-}\right)\right]-F_{2}-\Phi\left(F_{2}\right)\right] \mathcal{I}_{\tau_{2}=\tau_{F_{N}}^{2}}\right] \\
& =P\left(W_{t}, Y_{t} ; A\right),
\end{aligned}
$$


where again we have used the condition (B.5) for the second inequality, and the third inequality is obtained from (B.6).

Following similar steps for earlier subperiods we obtain that $\widetilde{P}\left(W_{t}, Y_{t}\right) \geq P\left(W_{t}, Y_{t} ; A\right)$. Since the policy $A$ is arbitrarily chosen, we have

$$
\widetilde{P}(W, Y) \geq \max _{A \in \mathcal{A}} P(W, Y ; A)=P(W, Y) .
$$

Finally, since the optimal strategies are attained, all the inequalities become equalities, so that:

$$
\widetilde{P}(W, Y)=P(W, Y ; A)=P(W, Y)
$$

when $A=A^{*}$, where

$$
A^{*} \in \mathcal{A}^{*}=\left\{\arg \max _{A \in \mathcal{A}} P(W, Y ; A)\right\}
$$

\section{B.6 Proof for Proposition 1}

Proof for the Existence and Boundedness Property. It is immediate to observe that the closed-form solution for $P^{*}(W, Y)$ with $(W, Y) \in \Sigma_{2}$ is bounded under Assumptions 1-2. It follows that $P(W, Y)$ is also bounded under Assumptions 1-2.

Lemma 1 shows that $W \leq P(W, Y)$. To see why $P(W, Y) \leq P^{*}(W, Y)$, let $P_{(1)}(W, Y)$ be the value function if the firm is allowed to issue equity freely without paying any external financing cost in the first round of financing. We then have:

$$
\begin{aligned}
P\left(W_{t}, Y_{t}\right)= & \mathbb{E}_{t}\left[e^{-r\left(\tau_{2}-t\right)}\left[P\left(W_{\tau_{2}-}+F_{2}, Y_{\tau_{2}-}\right)-F_{2}-\Phi\left(F_{2}\right)\right] \mathcal{I}_{\tau_{2}=\tau_{F}^{2}}+e^{-r\left(\tau_{2}-t\right)} W_{\tau_{2}} \mathcal{I}_{\tau_{2}=\tau_{L}^{2}}\right. \\
& \left.\quad+e^{-r\left(\tau_{2}-t\right)} P^{*}\left(W_{\tau_{2}}, Y_{\tau_{2}}\right) \mathcal{I}_{\tau_{2}=\tau_{U}^{2}}\right] \\
\leq & \mathbb{E}_{t}\left[e^{-r\left(\tau_{2}-t\right)}\left[P\left(W_{\tau_{2}-}+F_{2}, Y_{\tau_{2}-}\right)-F_{2}\right] \mathcal{I}_{\tau_{2}=\tau_{F}^{2}}+e^{-r\left(\tau_{2}-t\right)} W_{\tau_{2}} \mathcal{I}_{\tau_{2}=\tau_{L}^{2}}\right. \\
\quad & \left.\quad+e^{-r\left(\tau_{2}-t\right)} P^{*}\left(W_{\tau_{2}}, Y_{\tau_{2}}\right) \mathcal{I}_{\tau_{2}=\tau_{U}^{2}}\right] \\
= & P_{(1)}\left(W_{t}, Y_{t}\right) .
\end{aligned}
$$

Now, let $P_{(2)}(W, Y)$ be the value function if the firm has the opportunity to issue equity without any cost for the next two rounds. Then:

$$
\begin{aligned}
P_{(1)}\left(W_{t}, Y_{t}\right)= & \mathbb{E}_{t}\left[e^{-r\left(\tau_{2}-t\right)}\left[P\left(W_{\tau_{2}-}+F_{2}, Y_{\tau_{2}-}\right)-F_{2}\right] \mathcal{I}_{\tau_{2}=\tau_{F}^{2}}+e^{-r\left(\tau_{2}-t\right)} W_{\tau_{2}} \mathcal{I}_{\tau_{2}=\tau_{L}^{2}}\right. \\
& \left.\quad+e^{-r\left(\tau_{2}-t\right)} P^{*}\left(W_{\tau_{2}}, Y_{\tau_{2}}\right) \mathcal{I}_{\tau_{2}=\tau_{U}^{2}}\right] \\
\leq & \mathbb{E}_{t}\left[e^{-r\left(\tau_{2}-t\right)}\left[P_{(1)}\left(W_{\tau_{2}-}+F_{2}, Y_{\tau_{2}-}\right)-F\right] \mathcal{I}_{\tau_{2}=\tau_{F}^{2}}+e^{-r\left(\tau_{2}-t\right)} W_{\tau_{2}} \mathcal{I}_{\tau_{2}=\tau_{L}^{2}}\right. \\
& \left.\quad+e^{-r\left(\tau_{2}-t\right)} P^{*}\left(W_{\tau_{2}}, Y_{\tau_{2}}\right) \mathcal{I}_{\tau_{2}=\tau_{U}^{2}}\right] \\
= & P_{(2)}\left(W_{t}, Y_{t}\right) .
\end{aligned}
$$


Repeating the same argument, we have

$$
P(W, Y) \leq P_{(1)}(W, Y) \leq P_{(2)}(W, Y) \leq \ldots P_{(\infty)}(W, Y),
$$

where $P_{(\infty)}(W, Y)$ represents the value function for an infinite number of costless issues for given timing $\tau$. In other words, $P_{(\infty)}(W, Y)$ is the firm value when it is forever financial unconstrained for given timing $\tau$, so that $P_{(\infty)}(W, Y) \leq P^{*}(W, Y)$. It then follows that:

$$
P(W, Y) \leq P^{*}(W, Y) .
$$

Finally, we establish that $P(W, Y)$ exists under Assumption 2. Note first that the set of policies $\mathcal{A}$ is non-empty. Indeed, one policy $A \in \mathcal{A}$ is to immediately liquidate the firm at time $t, A=\left\{\left(\tau_{F}^{2}>t, \tau_{L}^{2}=t, F_{2}=0\right)\right\}$. The firm's payoff under this policy is $P\left(W_{t}, Y_{t} ; A\right)=W_{t}$. Therefore, there exists at least one policy $A^{*} \in \mathcal{A}$ such that $P\left(W_{t}, Y_{t} ; A^{*}\right)=\max _{A \in \mathcal{A}} P\left(W_{t}, Y_{t} ; A\right)$. Note that $P\left(W_{t}, Y_{t}\right)=$ $\max _{A \in \mathcal{A}} P\left(W_{t}, Y_{t} ; A\right)$, so that it exists and is given by $P\left(W_{t}, Y_{t}\right)=P\left(W_{t}, Y_{t} ; A^{*}\right)$.

Proof for the Monotonicity Property. First we show that $P(W, Y)$ is strictly increasing in $W$. For simplicity, define $Q\left(W_{t}, Y_{t} ; A\right)=P\left(W_{t}, Y_{t} ; A\right)-W$, and notice (13)we have the

$$
Q\left(W_{t}, Y_{t} ; A\right)=\mathbb{E}_{t}\left[e^{-r\left(\tau_{2}-t\right)}\left[-\Phi\left(F_{2}\right)\right] \mathcal{I}_{\tau_{2}=\tau_{F}^{2}}+e^{-r\left(\tau_{2}-t\right)} Q^{*}\left(Y_{\tau_{2}}\right) \mathcal{I}_{\tau_{2}=\tau_{U}^{2}}\right]
$$

and

$$
Q\left(W_{t}, Y_{t}\right)=\sup _{A \in \mathcal{A}} Q\left(W_{t}, Y_{t} ; A\right)
$$

Obviously, $P(W, Y)$ is strictly increasing in $W$ if $Q(W, Y)$ is increasing in $W$. Next, we prove $Q(W, Y)$ is increasing in $W$.

Let $W^{(1)}>W^{(2)}$, and $A_{1}^{*}, A_{2}^{*}$ be the optimal policies for the firm with initial wealth $W^{(1)}$ and $W^{(2)}$. Define $A_{2}^{*}=\left\{\tau_{L}^{2, *}, \tau_{F}^{2, *}, F_{2}^{2, *}\right\}$, and define $\tau_{U}^{2, *}$ as the time when the firm has accumulated sufficient liquidity to be permanently financially unconstrained.

For the firm with initial wealth $W^{(1)}$, a policy $A_{1}$ can be constructed with the same external financing decisions, including the financing time and amount, and the same liquidation time as policy $A_{2}^{*}$. Observe next that the dynamics of liquidity are then such that $W_{s}^{(1)} \geq W_{s}^{(2)}$ for $s>t$, path by path, if $W_{t}^{(1)}>W_{t}^{(2)}$, which implies $W_{\tau_{2}}^{(1)} \geq W_{\tau_{2}}^{(2)}=\Lambda_{2}$ if $\tau_{2}=\tau_{U}^{2, *}$.

Under this policy $A_{1}=A_{2}^{*}$ we then have:

$$
\begin{aligned}
Q\left(W_{t}^{(1)}, Y_{t} ; A_{2}^{*}\right) & =\mathbb{E}_{t}\left[e^{-r\left(\tau_{2}-t\right)}\left[-\Phi\left(F_{2}^{2, *}\right)\right] \mathcal{I}_{\tau_{2}=\tau_{F}^{2, *}}+e^{-r\left(\tau_{2}-t\right)} Q\left(W_{\tau_{2}}^{(1)}, Y_{\tau_{2}}\right) \mathcal{I}_{\tau_{2}=\tau_{U}^{2, *}}\right] \\
& =\mathbb{E}_{t}\left[e^{-r\left(\tau_{2}-t\right)}\left[-\Phi\left(F_{2}^{2, *}\right)\right] \mathcal{I}_{\tau_{2}=\tau_{F}^{2, *}}+e^{-r\left(\tau_{2}-t\right)} Q^{*}\left(Y_{\tau_{2}}\right) \mathcal{I}_{\tau_{2}=\tau_{U}^{2, *}}\right] \\
& =Q\left(W_{t}^{(2)}, Y_{t} ; A_{2}^{*}\right)=Q\left(W_{t}^{(2)}, Y_{t}\right) .
\end{aligned}
$$


Hence, we have

$$
\begin{aligned}
Q\left(W_{t}^{(1)}, Y_{t}\right) & =Q\left(W_{t}^{(1)}, Y_{t} ; A_{1}^{*}\right) \geq Q\left(W_{t}^{(1)}, Y_{t} ; A_{1}\right)=Q\left(W_{t}^{(1)}, Y_{t} ; A_{2}^{*}\right) \\
& =Q\left(W_{t}^{(2)}, Y_{t} ; A_{2}^{*}\right)=Q\left(W_{t}^{(2)}, Y_{t}\right) .
\end{aligned}
$$

That is $Q(W, Y)$ is increasing in $W$ and $P(W, Y)=Q(W, Y)+W$ is strictly increasing in $W$.

Second, we show that $P(W, Y)$ is strictly increasing in $Y$ for $Y \geq \underline{Y}(W)$, where $\underline{Y}(W)$ denotes the liquidation boundary. That is $P\left(W, Y^{(1)}\right)>P\left(W, Y^{(2)}\right)$ if $Y^{(1)}>$ $Y^{(2)} \geq \underline{Y}(W)$. We can use the same argument as for the monotonicity in $W$. Again, defining $A_{1}^{*}$ and $A_{2}^{*}$ as above, we can take $A_{1}=A_{2}^{*}$. Furthermore, let $W^{(1)}$ and $W^{(2)}$ denote the liquidity of the firm with initial earnings fundamentals $Y^{(1)}$ and $Y^{(2)}$ respectively. Observe next that the dynamics of liquidity are then such that $W_{s}^{(1)}>W_{s}^{(2)}$ for $s>t$, path by path, if $W_{t}^{(1)}=W_{t}^{(2)}$. Also, $Y_{t}^{(1)}>Y_{t}^{(2)} \geq \underline{Y}\left(W_{t}\right)$ implies that $W_{\tau_{2}}^{(1)} \geq W_{\tau_{2}}^{(2)}$ if $\tau_{2}=\min \left\{\tau_{L}^{2, *}, \tau_{U}^{2, *}\right\}$.

Since $P^{*}(W, Y)$ is always strictly increasing in $W$ and $Y$, the terminal payoff with initial earnings fundamentals $Y^{(1)}$ is always higher than with initial earnings fundamentals $Y^{(2)}$, path by path, under the same policy $A_{2}^{*}$, so that:

$$
P\left(W, Y^{(1)} ; A_{1}\right)=P\left(W, Y^{(1)} ; A_{2}^{*}\right)>P\left(W, Y^{(2)} ; A_{2}^{*}\right)=P\left(W, Y^{(2)}\right) .
$$

Furthermore, $P\left(W, Y^{(1)}\right)=P\left(W, Y^{(1)} ; A_{1}^{*}\right) \geq P\left(W, Y^{(1)} ; A_{1}\right)$, so that:

$$
P\left(W, Y^{(1)}\right)>P\left(W, Y^{(2)}\right) \quad \text { for } \quad Y^{(1)}>Y^{(2)} \geq \underline{Y}(W) .
$$

Proof for the Uniqueness Property. Let $\Sigma_{P}$ denote the interior region where $\mathcal{L}_{2} P=0$ :

$$
\Sigma_{P}=\left\{(W, Y) \mid 0<W<\Lambda_{2}, \underline{Y}(W)<Y<\infty\right\} .
$$

Let $\Sigma_{P}^{\prime}$ denote the boundary of $\Sigma_{P}$ :

$$
\Sigma_{P}^{\prime}=\left\{(W, Y) \mid W=\left\{0, \Lambda_{2}\right\}, \underline{Y}(W) \leq Y<\infty\right\} \cup\left\{(W, Y) \mid 0 \leq W \leq \Lambda_{2}, Y=\underline{Y}(W)\right\} .
$$

We first show that the solution is unique for a given region $\Sigma_{P}$ and then show that $\Sigma_{P}$ is unique. Finally, we show the the solution is unique on the whole region $\widehat{\Sigma}_{2}=$ $\Sigma_{P} \cup \Sigma_{P}^{\prime}$ 
Suppose by contradiction that for a given region $\Sigma_{P}$ where $\mathcal{L}_{2} P=0$, there are two solutions $P^{(1)}(W, Y)$ and $P^{(2)}(W, Y)$ which satisfy:

$$
\begin{aligned}
0= & \left(r W+m_{2} Y-Z_{2}\right) P_{W}^{(1)}(W, Y)+\mu Y P_{Y}^{(1)}(W, Y)+\frac{\sigma^{2} Y^{2} P_{Y Y}^{(1)}(W, Y)}{2}-r P^{(1)}(W, Y), \\
& \text { and } \\
0= & \left(r W+m_{2} Y-Z_{2}\right) P_{W}^{(2)}(W, Y)+\mu Y P_{Y}^{(2)}(W, Y)+\frac{\sigma^{2} Y^{2} P_{Y Y}^{(2)}(W, Y)}{2}-r P^{(2)}(W, Y),
\end{aligned}
$$

in the region $\Sigma_{P}$. Let $\widehat{P}(W, Y)=P^{(1)}(W, Y)-P^{(2)}(W, Y)$. Then $\widehat{P}(W, Y)$ also satisfies

$$
0=\left(r W+m_{2} Y-Z_{2}\right) \widehat{P}_{W}(W, Y)+\mu Y \widehat{P}_{Y}(W, Y)+\frac{\sigma^{2} Y^{2} \widehat{P}_{Y Y}(W, Y)}{2}-r \widehat{P}(W, Y),
$$

in region $\Sigma_{P}$. Let

$$
(\widehat{W}, \widehat{Y}) \in \arg \max _{(W, Y) \in \Sigma_{P} \cup \Sigma_{P}^{\prime}} \widehat{P}(W, Y)
$$

If $(\widehat{W}, \widehat{Y}) \in \Sigma_{P}$, we have $\widehat{P}_{W}(\widehat{W}, \widehat{Y})=0, \widehat{P}_{Y}(\widehat{W}, \widehat{Y})=0$ and $\widehat{P}_{Y Y}(\widehat{W}, \widehat{Y}) \leq 0$.

Under Assumption 2, we also have:

$$
r \widehat{P}(\widehat{W}, \widehat{Y})=\frac{\sigma^{2} \widehat{Y}^{2} \widehat{P}_{Y Y}(\widehat{W}, \widehat{Y})}{2} \leq 0
$$

Therefore, we have

$$
\widehat{P}(\widehat{W}, \widehat{Y}) \leq 0, \quad \text { if } \quad(\widehat{W}, \widehat{Y}) \in \Sigma_{P}
$$

Note that $(W, \underline{Y}(W)) \in \Sigma_{P}^{\prime}$, we have

$$
\begin{aligned}
\max _{(W, Y) \in \Sigma_{P} \cup \Sigma_{P}^{\prime}} \widehat{P}(W, Y) & \geq \max _{(W, Y) \in \Sigma_{P}^{\prime}} \widehat{P}(W, Y) \geq P^{(1)}(W, \underline{Y}(W))-P^{(2)}(W, \underline{Y}(W)) \\
& =W-W=0 .
\end{aligned}
$$

That is

$$
\widehat{P}(\widehat{W}, \widehat{Y}) \geq 0, \quad \text { for } \quad(\widehat{W}, \widehat{Y}) \in \Sigma_{P} \cup \Sigma_{P}^{\prime}
$$

Combining (B.15) and (B.16), we have

$$
\widehat{P}(\widehat{W}, \widehat{Y}) \equiv 0, \quad \text { if } \quad(\widehat{W}, \widehat{Y}) \in \Sigma_{P}
$$

Next, we prove that $\widehat{P}(\widehat{W}, \widehat{Y}) \equiv 0$ if $(\widehat{W}, \widehat{Y}) \in \Sigma_{P}^{\prime}$. By using closed-form value functions on the boundaries: $Y=\underline{Y}(W)$ and $W=\Lambda_{2}$, we have

$$
\begin{gathered}
\widehat{P}(\widehat{W}, \widehat{Y})=0, \text { if }(\widehat{W}, \widehat{Y}) \in\left\{(W, Y) \mid W=\Lambda_{2}, \underline{Y}(W) \leq Y<\infty\right\} \\
\cup\left\{(W, Y) \mid 0 \leq W \leq \Lambda_{2}, Y=\underline{Y}(W)\right\} .
\end{gathered}
$$


Next we show $\widehat{P}(\widehat{W}, \widehat{Y})=0$, if $(\widehat{W}, \widehat{Y}) \in\{(W, Y) \mid W=0, \underline{Y}(W) \leq Y<\infty\}$. First, note that $\widehat{P}_{W}(\widehat{W}, \widehat{Y}) \leq 0, \widehat{P}_{Y}(\widehat{W}, \widehat{Y})=0$ and $\widehat{P}_{Y Y}(\widehat{W}, \widehat{Y}) \leq 0$ if $\widehat{W}=0$, and $\frac{Z_{2}}{m_{2}} \leq \widehat{Y}<\infty$. Therefore, we have $r \widehat{P}(0, \widehat{Y})=\left(m_{2} \widehat{Y}-Z_{2}\right) \widehat{P}_{W}(W, Y)+\frac{\sigma^{2} \widehat{Y}^{2} \widehat{P}_{Y Y}(0, \widehat{Y})}{2} \leq 0$ if $\frac{Z_{2}}{m_{2}} \leq \widehat{Y}<\infty$. Then using (B.16), we have

$$
\widehat{P}(0, \widehat{Y})=0, \quad \text { if } \quad \frac{Z_{2}}{m_{2}} \leq \widehat{Y}<\infty
$$

Finally, let $F_{2}^{(1)}$ and $F_{2}^{(2)}$ denote the optimal equity issues in that $P^{(1)}(0, \widehat{Y})=$ $\sup _{F_{2}>0} P^{(1)}\left(F_{2}, \widehat{Y}\right)-F_{2}-\Phi\left(F_{2}\right)$ and $P^{(2)}(0, \widehat{Y})=\sup _{F_{2}>0} P^{(2)}\left(F_{2}, \widehat{Y}\right)-F_{2}-\Phi\left(F_{2}\right)$. We thus have

$$
\begin{aligned}
\widehat{P}(0, \widehat{Y}) & =P^{(1)}(0, \widehat{Y})-P^{(2)}(0, \widehat{Y}) \\
& =\left(P^{(1)}\left(F_{2}^{(1)}, \widehat{Y}\right)-F_{2}^{(1)}-\Phi\left(F_{2}^{(1)}\right)\right)-\left(P^{(2)}\left(F_{2}^{(2)}, \widehat{Y}\right)-F_{2}^{(2)}-\Phi\left(F_{2}^{(2)}\right)\right) \\
& \leq\left(P^{(1)}\left(F_{2}^{(1)}, \widehat{Y}\right)-F_{2}^{(1)}-\Phi\left(F_{2}^{(1)}\right)\right)-\left(P^{(2)}\left(F_{2}^{(1)}, \widehat{Y}\right)-F_{2}^{(1)}-\Phi\left(F_{2}^{(1)}\right)\right) \\
& =\widehat{P}\left(F_{2}^{(1)}, \widehat{Y}\right) .
\end{aligned}
$$

This is obviously a contradiction unless $\widehat{P}(W, Y) \equiv 0$. That is $\widehat{P}(0, \widehat{Y})=0$ if $\underline{Y}(0) \leq \widehat{Y}<\frac{Z_{2}}{m_{2}}$. Together with (B.19) and (B.18), we conclude that

$$
\widehat{P}(\widehat{W}, \widehat{Y}) \equiv 0, \quad \text { if } \quad(\widehat{W}, \widehat{Y}) \in \Sigma_{P}^{\prime}
$$

Further by combining (B.17) and (B.21), we conclude

$$
\widehat{P}(\widehat{W}, \widehat{Y}) \equiv 0 \quad \text { for } \quad(W, Y) \in \Sigma_{P} \cup \Sigma_{P}^{\prime}
$$

This implies that

$$
P^{(1)}(W, Y) \leq P^{(2)}(W, Y) \quad \text { for } \quad(W, Y) \in \Sigma_{P} \cup \Sigma_{P}^{\prime}
$$

Since the entire analysis also holds if we switch $P^{(1)}(W, Y)$ with $P^{(2)}(W, Y)$, in that

$$
P^{(2)}(W, Y) \leq P^{(1)}(W, Y) \quad \text { for } \quad(W, Y) \in \Sigma_{P} \cup \Sigma_{P}^{\prime},
$$

we thus conclude that

$$
P^{(2)}(W, Y) \equiv P^{(1)}(W, Y) \quad \text { for } \quad(W, Y) \in \Sigma_{P} \cup \Sigma_{P}^{\prime}
$$

We next show that the region $\Sigma_{P}$ is unique, which is equivalent to showing that the boundary $\Sigma_{P}^{\prime}$ is unique. From the definition of $\Sigma_{P}^{\prime}$, we only need to show that $\underline{Y}(W)$ is unique for $0 \leq W \leq \Lambda_{2}$. Suppose that there are two liquidation boundaries 
$\underline{Y}^{(1)}(W)$ and $\underline{Y}^{(2)}(W)$, with $\underline{Y}^{(1)}(W) \geq \underline{Y}^{(2)}(W)$ for some $W$, and let $P^{(1)}(W, Y)$ and $P^{(2)}(W, Y)$ be the corresponding solutions. We then have:

$$
W=P^{(1)}\left(W, \underline{Y}^{(1)}(W)\right) \geq P^{(2)}\left(W, \underline{Y}^{(1)}(W)\right) \geq W=P^{(2)}\left(W, \underline{Y}^{(2)}(W)\right),
$$

where the first inequality follows from the optimality of the liquidation decision and the second inequality is obtained from (15). It follows that $P^{(2)}\left(W, \underline{Y}^{(1)}(W)\right)=$ $P^{(2)}\left(W, \underline{Y}^{(2)}(W)\right)$. Moreover, the value function is increasing in both $W$ and $Y$ so that: $\underline{Y}^{(1)}(W)=\underline{Y}^{(2)}(W)$. Finally, we show that the solution is unique on the remaining liquidation region $\Sigma_{2}^{\prime}=\Sigma_{2}-\left(\Sigma_{P} \cup \Sigma_{P}^{\prime}\right)$. From the definition of the regions $\Sigma_{P}$ and $\Sigma_{P}^{\prime}$, and total region $\Sigma_{2}$, we have that

$$
\Sigma_{2}^{\prime}=\left\{(W, Y) \mid 0 \leq W \leq \Lambda_{2}, 0 \leq Y<\underline{Y}(W)\right\} .
$$

Since $\underline{Y}(W)$ is unique it follows that $\Sigma_{2}^{\prime}$ is also unique. And in the liquidation region $\Sigma_{2}^{\prime}$ the value function is $P(W, Y) \equiv W$. In sum, the solution of $P(W, Y)$ in the region $\Sigma_{2}$ is unique.

\section{B.7 Proof for Proposition 2}

Proof for Monotonicity Property of Liquidation Boundary. Here, we want to show that the optimal liquidation boundary, $\underline{Y}(W)$, is decreasing in $W$ in the mature phase. Let $\underline{Y}\left(W^{(1)}\right)$ and $\underline{Y}\left(W^{(2)}\right)$ denote the optimal liquidation boundary with liquidity $W^{(1)}$ and $W^{(2)}$, where $W^{(1)}<W^{(2)}$. Recall that $Q(W, Y)=P(W, Y)-$ $W$ is increasing in $W$ as shown in Proposition 1, we have $0=Q\left(W^{(1)}, \underline{Y}\left(W^{(1)}\right)\right) \leq$ $Q\left(W^{(2)}, \underline{Y}\left(W^{(1)}\right)\right)$. In addition, $P(W, Y)$ is strictly increasing in $Y$ before liquidation, as shown in Proposition 1, which implies that $Q(W, Y)=P(W, Y)-W$ is also strictly increasing in $Y$ for $Y \geq \underline{Y}(W)$. Hence, we have $\underline{Y}\left(W^{(1)}\right) \geq \underline{Y}\left(W^{(2)}\right)$, otherwise $0=Q\left(W^{(1)}, \underline{Y}\left(W^{(1)}\right)\right) \leq Q\left(W^{(2)}, \underline{Y}\left(W^{(1)}\right)\right)<Q\left(W^{(2)}, \underline{Y}\left(W^{(2)}\right)\right)=0$, which is a contradiction. That is, $\underline{Y}\left(W^{(1)}\right) \geq \underline{Y}\left(W^{(2)}\right)$ if $W^{(1)}<W^{(2)}$, which establishes that the optimal liquidation boundary is decreasing in $W$ in the mature phase.

Proof for Delaying Costly Equity Issuance. First, we need to show that the firm has to issue equity if $W=0$, for $Y$ such that $\underline{Y}(0)<Y<\frac{Z_{2}}{m_{2}}$, to be able to continue operations. We assume that $F^{*}(0)$ is the optimal amount of equity issuance, which implies that $P(0, Y)=P\left(F^{*}(0), Y\right)-F^{*}(0)-\Phi\left(F^{*}(0)\right)$. This also implies that $\left.P_{W}(0, Y)>1+\Phi^{* \prime}(0)\right)=1+\phi_{1}$, otherwise $F^{*}(0)=0$ and the firm defaults immediately, which is a contradiction for $\underline{Y}(0)<Y<\frac{Z_{2}}{m_{2}}$. Denote $M=F^{*}(0)$, we have that $P_{W}(W, Y)>1+\phi_{1}$ for $0<W<M$ and $P_{W}(M, Y)=1+\phi_{1}$, otherwise $F^{*}(0)$ is not the optimal amount of equity issuance. 
To show that the firm will delay costly equity issuance until entirely exhausting its cash, we only need to show that $P(W, Y)>\sup _{F_{2} \geq 0} P\left(W+F_{2}, Y\right)-F_{2}-\Phi\left(F_{2}\right)$ for $W>0$. First, since $P_{W}(W, Y) \leq 1+\phi_{1}$ for $W \geq M$, the firm has no incentive to issue equity because $P(W, Y) \geq P\left(W+F_{2}, Y\right)-F_{2}-\phi_{1} F_{2}>P\left(W+F_{2}, Y\right)-F_{2}-\Phi\left(F_{2}\right)$ for $W \geq M$. Next, we show that $P(W, Y)>\sup _{F_{2} \geq 0} P\left(W+F_{2}, Y\right)-F_{2}-\Phi\left(F_{2}\right)$ for $0<W<M$. We define $F^{*}(W)$ as the optimal amount of equity issuance if the firm chooses equity issuance at $W$ with $0<W<M$ for given $Y$, which implies that $\sup _{F_{2} \geq 0} P\left(W+F_{2}, Y\right)-F-\Phi\left(F_{2}\right)=P\left(W+F_{2}^{*}(W), Y\right)-F_{2}^{*}(W)-\Phi\left(F_{2}^{*}(W)\right)$ and $M=W+F_{2}^{*}(W)$. We then have

$$
\begin{aligned}
& P(W, Y)-\left(P\left(W+F_{2}^{*}(W), Y\right)-F_{2}^{*}(W)-\Phi\left(F_{2}^{*}(W)\right)\right) \\
& \quad=P(W, Y)-\left(P(M, Y)-F^{*}(W)-\Phi\left(F_{2}^{*}(W)\right)\right) \\
& \quad=P(W, Y)-W-\left(P(M, Y)-M-\Phi\left(F_{2}^{*}(W)\right)\right) \\
& \quad=Q(W, Y)-Q(M, Y)+\Phi(M-W) .
\end{aligned}
$$

Recall that $P(0, Y)=P\left(F_{2}^{*}(0), Y\right)-F_{2}^{*}(0)-\Phi\left(F_{2}^{*}(0)\right)$ and $M=F_{2}^{*}(0)$, and $Q(0, Y)=$ $Q\left(F_{2}^{*}(0), Y\right)-\Phi\left(F_{2}^{*}(0)\right)=Q(M, Y)-\Phi(M)$, so that

$$
\begin{aligned}
& P(W, Y)-\left(P\left(W+F_{2}^{*}(W), Y\right)-F_{2}^{*}(W)-\Phi\left(F_{2}^{*}(W)\right)\right) \\
& \quad=Q(W, Y)-Q(0, Y)+\Phi(M-W)-\Phi(M) \\
& \quad=Q(W, Y)-Q(0, Y)-\phi_{1} W \\
& \quad>\phi_{1} W-\phi_{1} W=0,
\end{aligned}
$$

where the above inequality follows from $P_{W}(W, Y)>1+\phi_{1}$ for $0<W<M$, which implies that $Q_{W}(W, Y)>\phi_{1}$ for $0<W<M$. Finally, $P(W, Y)-\left(P\left(W+F_{2}^{*}(W), Y\right)-\right.$ $\left.F_{2}^{*}(W)-\Phi\left(F_{2}^{*}(W)\right)\right)>0$ implies that the firm has no incentive to issue equity for $0<W<M$. In sum, we have shown that the firm has no incentive to issue equity for $W>0$, which implies that the firm will delay costly equity issuance until entirely exhausting its cash.

\section{Proofs for the general case in the growth phase}

\section{C.1 Proof for Lemma 5}

For any given time $t$, we can construct a control policy $\widehat{A} \in \mathcal{A}$ with $\widehat{A}=\left\{\left(\tau_{i}>\right.\right.$ $\left.\left.t, \tau_{L}^{1}=t, \tau_{F}^{1}>t, F_{1}\right)\right\}$ such that the firm is liquidated immediately at time $t$ in the growth phase. Under this policy, we have

$$
G\left(W_{t}, Y_{t} ; \widehat{A}\right)=W_{t} .
$$


Since $G\left(W_{t}, Y_{t}\right)=\sup _{A \in \mathcal{A}} G\left(W_{t}, Y_{t} ; A\right) \geq G\left(W_{t}, Y_{t} ; \widehat{A}\right)$, it immediately follows that $G(W, Y) \geq W$.

\section{C.2 Proof for Lemma 6}

For any given time $t$, we can construct a control policy $\widehat{A} \in \mathcal{A}$ with $\widehat{A}=$ $\left\{\left(\tau_{i}>t, \tau_{L}^{1}>t, \tau_{F}^{1}=t, F_{1} \geq 0\right)\right\}$ such that the firm issues equity at time $t$, and for any $s \geq t$ we assume that the firm takes the optimal investment, liquidation and financing decisions, including financing timing and the choice of the optimal amount $F_{1}$. Following the above policy, the (optimal) value function at time $t$ is $G\left(W_{t-}+F_{1}, Y_{t-}\right)-F_{1}-\Phi\left(F_{1}\right)$. By the continuity of value function upon the equity issue, we have

$$
G\left(W_{t-}, Y_{t-} ; \widehat{A}\right)=G\left(W_{t-}+F_{1}, Y_{t-}\right)-F_{1}-\Phi\left(F_{1}\right) .
$$

Since the time of equity issue for $\tau_{F}^{1}=t$ may be not optimal, we have

$$
\sup _{A \in \mathcal{A}} G\left(W_{t-}, Y_{t-} ; A\right) \geq \sup _{F_{1} \geq 0} G\left(W_{t-}, Y_{t-} ; \widehat{A}\right)=\sup _{F_{1} \geq 0} G\left(W_{t-}+F_{1}, Y_{t-}\right)-F_{1}-\Phi\left(F_{1}\right) .
$$

Recall that $G\left(W_{t-}, Y_{t-}\right)=\sup _{A \in \mathcal{A}} G\left(W_{t-}, Y_{t-} ; A\right)$, so that we have $G\left(W_{t-}, Y_{t-}\right) \geq$ $\sup _{F_{1} \geq 0} G\left(W_{t-}+F_{1}, Y_{t-}\right)-F_{1}-\Phi\left(F_{1}\right)$.

\section{C.3 Proof for Lemma 7}

For any given time $t$, we can construct a control policy $\widehat{A} \in \mathcal{A}$ with $\widehat{A}=\left\{\left(\tau_{i}=\right.\right.$ $\left.\left.t, \tau_{L}^{1}>t, \tau_{F}^{1}=t, F_{1} \geq 0\right)\right\}$ such that the firm exercises its growth option and/or issues equity at time $t$, and for any $s \geq t$ it is in the mature phase. Following the above policy, the (optimal) value function at time $t$ is $P\left(W_{t-}+F_{1}-I, Y_{t-}\right)-F_{1}-\Phi\left(F_{1}\right)$. By continuity of value function upon the equity issue, we have

$$
G\left(W_{t-}, Y_{t-} ; \widehat{A}\right)=P\left(W_{t-}+F_{1}-I, Y_{t-}\right)-F_{1}-\Phi\left(F_{1}\right) .
$$

Since the time of the equity issue $\tau_{i}=t$ may be not optimal, we have

$$
\sup _{A \in \mathcal{A}} G\left(W_{t-}, Y_{t-} ; A\right) \geq \sup _{F_{1} \geq 0} G\left(W_{t-}, Y_{t-} ; \widehat{A}\right)=\sup _{F_{1} \geq 0} P\left(W_{t-}+F_{1}-I, Y_{t-}\right)-F_{1}-\Phi\left(F_{1}\right) .
$$

Recall that $G\left(W_{t-}, Y_{t-}\right)=\sup _{A \in \mathcal{A}} G\left(W_{t-}, Y_{t-} ; A\right)$, so that we have $G\left(W_{t-}, Y_{t-}\right) \geq$ $\sup _{F_{1} \geq 0} P\left(W_{t-}+F_{1}-I, Y_{t-}\right)-F_{1}-\Phi\left(F_{1}\right)$. 


\section{C.4 Proof for Lemma 8}

We assume that the current time is $t$, and that $Y_{a, 1}^{*}<Y_{t} \leq Y_{i}^{*}$, and denote $\tau_{L}^{*}=\inf \left\{s \mid Y_{s} \leq Y_{a, 1}^{*}\right\}$ and $\tau_{i}^{*}=\inf \left\{s \mid Y_{s} \geq Y_{i}^{*}\right\}$. Obviously, $\tau_{L}^{*}$ is the optimal first-best liquidation time and $\tau_{i}^{*}$ is the first-best optimal investment time. Note that $\Lambda_{1}=\frac{Z_{1}-m_{1} Y_{a, 1}}{r}$ and $W_{t} \geq \Lambda_{1}$ imply that the drift of $W$ in the growth phase $\left(r W_{s}+m_{1} Y_{s}-Z_{1}\right)$ is always positive for $t \leq s<\tau$, where $\tau=\left(\tau_{L}^{*} \bigwedge \tau_{i}^{*}\right)$, which means that $W_{s} \geq W_{t}>0$ for $t \leq s<\tau$, and the firm will never use external financing by issuing equity before liquidation or exercising its growth option. In addition, we have that $W_{s} \geq W_{t}>I+\Lambda_{2}$ since $\left(r W_{s}+m_{1} Y_{s}-Z_{1}\right) \geq 0$ for $t \leq s<\tau$. Hence, we can construct a control policy $\widehat{A} \in \mathcal{A}$ such that $\widehat{A}=\left\{\left(\tau_{i}=\tau_{i}^{*}, \tau_{L}^{1}=\tau_{L}^{*}, \tau_{F}^{1}=\infty, F_{1}=0\right)\right\}$.

From (29) we have

$$
G\left(W_{t}, Y_{t} ; A\right)=\mathbb{E}_{t}\left[e^{-r\left(\tau_{i}^{*}-t\right)} P\left(W_{\tau_{i}^{*}-}-I, Y_{\tau_{i}^{*}-}\right) \mathcal{I}_{\tau_{L}^{*}>\tau_{i}^{*}}+e^{-r\left(\tau_{L}^{*}-t\right)} W_{\tau_{L}^{*}} \mathcal{I}_{\tau_{L}^{*}<\tau_{i}^{*}}\right] .
$$

Note that $W_{\tau_{i}^{*}-}>I+\Lambda_{2}$, therefore by Lemma (3) and Theorem 1 we have

$$
P\left(W_{\tau_{i}^{*}-}-I, Y_{\tau_{i}^{*}-}\right)=W_{\tau_{i}^{*}-}-I+Q\left(Y_{\tau_{i}^{*}-}\right)=W_{\tau_{i}^{*}-}+H^{*}\left(Y_{\tau_{i}^{*}-}\right)=G^{*}\left(W_{\tau_{i}^{*}-}, Y_{\tau_{i}^{*}-}\right) .
$$

Note also that $Y_{\tau_{L}^{*}}=Y_{a, 1}^{*}$, so that by Theorem 1 we have

$$
W_{\tau_{L}^{*}}=W_{\tau_{L}^{*}}+0=W_{\tau_{L}^{*}}+H^{*}\left(Y_{\tau_{L}^{*}}\right)=G^{*}\left(W_{\tau_{L}^{*}}, Y_{\tau_{L}^{*}}\right) .
$$

Hence, we could rewrite (C.1) as

$$
\begin{aligned}
G\left(W_{t}, Y_{t} ; A\right) & =\mathbb{E}_{t}\left[e^{-r\left(\tau_{i}^{*}-t\right)} G^{*}\left(W_{\tau_{i}^{*}-}, Y_{\tau_{i}^{*}-}\right) \mathcal{I}_{\tau_{L}^{*}>\tau_{i}^{*}}+e^{-r\left(\tau_{L}^{*}-t\right)} G^{*}\left(W_{\tau_{L}^{*}}, Y_{\tau_{L}^{*}}\right) \mathcal{I}_{\tau_{L}^{*}<\tau_{i}^{*}}\right] \\
& =\mathbb{E}_{t}\left[e^{-r(\tau-t)} G^{*}\left(W_{\tau-}, Y_{\tau-}\right)\right] \\
& =G^{*}\left(W_{t}, Y_{t}\right) .
\end{aligned}
$$

Finally, since $G(W, Y)=\sup _{A \in \mathcal{A}} G(W, Y ; A)$, we have $G(W, Y) \geq G^{*}(W, Y)$ for $W \geq \max \left\{\Lambda_{1}, \Lambda_{2}+I\right\}$. And, following the boundedness property of $G(W, Y)$ as shown in Proposition 3, we have $G(W, Y)=G^{*}(W, Y)$ for $W \geq \max \left\{\Lambda_{1}, \Lambda_{2}+I\right\}$.

\section{C.5 Proof for Lemma 9}

Assume that for any given time $t, W_{t} \geq 0, Y_{t} \rightarrow \infty$, and that the firm always takes optimal decisions. Consider a tiny time period $(t, t+\Delta t)$ with $\Delta t>0$. During this period the firm abandons or issues equity with zero probability, since $Y_{t} \rightarrow \infty$. Hence, we have $W_{t+\Delta t}>\max \left\{\Lambda_{1}, \Lambda_{2}+I\right\}$ with probability one when $Y_{t} \rightarrow \infty$, which implies that $G\left(W_{t+\Delta t}, Y_{t+\Delta t}\right)=G^{*}\left(W_{t+\Delta t}, Y_{t+\Delta t}\right)$ with probability one, by Lemma 8 . From (29) under optimal decisions we have

$$
G\left(W_{t}, Y_{t}\right)=\mathbb{E}_{t}\left[e^{-r(t+\Delta t-t)} G\left(W_{t+\Delta t}, Y_{t+\Delta t}\right)\right]=\mathbb{E}_{t}\left[e^{-r \Delta t} G^{*}\left(W_{t+\Delta t}, Y_{t+\Delta t}\right)\right] .
$$


Since $Y_{t} \rightarrow \infty$, the above equation holds for any $\Delta>0$, and by taking $\Delta \rightarrow 0$, we have

$$
G\left(W_{t}, Y_{t}\right)=\lim _{\Delta \rightarrow 0} \mathbb{E}_{t}\left[e^{-r \Delta t} G^{*}\left(W_{t+\Delta t}, Y_{t+\Delta t}\right)\right]=\mathbb{E}_{t}\left[G^{*}\left(W_{t}, Y_{t}\right)\right]=G^{*}\left(W_{t}, Y_{t}\right) .
$$

\section{C.6 Proof for Theorem 3}

Verification. We establish that any piecewise- $\mathcal{C}^{2}$ function, which is a solution to the equation (40), associated to problem (29), is a majorant of the value function $G$.

Proposition 6 (Verification 2) Suppose that a positive function $\widetilde{G}$ exists which is piecewise- $\mathcal{C}^{2}$ on the region $\Sigma_{1}$ with bounded first derivatives and such that for all $(W, Y) \in \Sigma_{1}$,

$$
\begin{aligned}
\mathcal{L}_{1} \widetilde{G} & \leq 0, \\
\widetilde{G}(W, Y) & \geq W, \\
\widetilde{G}(W, Y) & \geq \sup _{F_{1} \geq 0} P\left(W+F_{1}-I, Y\right)-F_{1}-\Phi\left(F_{1}\right), \\
\widetilde{G}(W, Y) & \geq \sup _{F_{1} \geq 0} \widetilde{G}\left(W+F_{1}, Y\right)-F_{1}-\Phi\left(F_{1}\right),
\end{aligned}
$$

with boundary conditions $\widetilde{G}(W, Y)=G^{*}(W, Y)$ for $W=\max \left\{\Lambda_{1}, \Lambda_{2}+I\right\}$ or $Y \rightarrow \infty$. Then $\widetilde{G}(W, Y)=G(W, Y)$ for all $(W, Y) \in \Sigma_{1}$.

Proof. We first show that $\widetilde{G}(W, Y) \geq G(W, Y)$. Notice that $A$ is a control policy in problem (29), where $A \in \mathcal{A}$ the set of all policies. Then, by definition, we have (30). If we can show that $\widetilde{G}(W, Y) \geq G(W, Y ; A)$ for any control policy $A \in \mathcal{A}$, then it follows that $\widetilde{G}(W, Y) \geq G(W, Y)$.

Using the generalized Ito's formula (Dellacherie and Meyer (1980), Theorem VIII25 and Remark c, p. 349), we can write:

$$
\begin{aligned}
e^{-r\left(\tau_{1}-t\right)} \widetilde{G}\left(W_{\tau_{1}-}, Y_{\tau-}\right)=\widetilde{G}( & \left.W_{t}, Y_{t}\right)+\int_{t}^{\tau_{1}-} e^{-r(s-t)} \mathcal{L}_{2} \widetilde{G}\left(W_{s}, Y_{s}\right) d s \\
& +\int_{t}^{\tau_{1}-} e^{-r(s-t)} \widetilde{G}_{Y}\left(W_{s}, Y_{s}\right) \sigma Y_{s} d B_{s} .
\end{aligned}
$$

Since $\widetilde{G}$ satisfies (C.3), the second term of the right hand side is non-positive. Moreover, since the first derivative of $\widetilde{G}$ is bounded, the third term is a square integrable martingale. Taking expectations, we then get:

$$
\mathbb{E}_{t}\left[e^{-r\left(\tau_{1}-t\right)} \widetilde{G}\left(W_{\tau_{1}-}, Y_{\tau_{1}-}\right)\right] \leq \widetilde{G}\left(W_{t}, Y_{t}\right) .
$$


Suppose that the firm issues equity $N$ times from time 0 to time $\tau_{L}^{1} \wedge \tau_{U}^{1}$, and divide the time interval $\left(0, \tau_{L}^{1} \wedge \tau_{U}^{1}\right)$ into $N+1$ subperiods. We define $\tau_{F_{n}}^{1}$ as the time of the $n$-th round of external financing, where $1 \leq n \leq N$. During the last period $\left(\tau_{F_{N}}^{1}, \tau_{L}^{1} \wedge \tau_{U}^{1}\right)$ we then have:

$$
\begin{aligned}
\widetilde{G}\left(W_{t}, Y_{t}\right) & \geq \mathbb{E}_{t}\left[e^{-r\left(\tau_{1}-t\right)} \widetilde{G}\left(W_{\tau_{1}-}, Y_{\tau_{1}-}\right)\right] \\
& =\mathbb{E}_{t}\left[e^{-r\left(\tau_{1}-t\right)} \widetilde{G}\left(W_{\tau_{1}}, Y_{\tau_{1}}\right) \mathcal{I}_{\tau_{1}=\tau_{L}^{1}}+e^{-r\left(\tau_{1}-t\right)} G^{*}\left(W_{\tau_{1}}, Y_{\tau_{1}}\right) \mathcal{I}_{\tau_{1}=\tau_{U}^{1}}\right] \\
& \geq \mathbb{E}_{t}\left[e^{-r\left(\tau_{1}-t\right)} W_{\tau_{1}} \mathcal{I}_{\tau_{1}=\tau_{L}^{1}}+e^{-r\left(\tau_{1}-t\right)} G^{*}\left(W_{\tau_{1}}, Y_{\tau_{1}}\right) \mathcal{I}_{\tau_{1}=\tau_{U}^{1}}\right] \\
& =G\left(W_{t}, Y_{t} ; A\right)
\end{aligned}
$$

where we have used the condition (C.4) for the second inequality.

During the $N$-th external financing subperiod $\left(\tau_{F_{N-1}}^{1}, \tau_{1}\right)$ we have in turn:

$$
\begin{aligned}
& \widetilde{G}\left(W_{t}, Y_{t}\right) \geq \mathbb{E}_{t}\left[e^{-r\left(\tau_{1}-t\right)} \widetilde{G}\left(W_{\tau_{1}-}, Y_{\tau_{1}-}\right)\right] \\
& =\mathbb{E}_{t}\left[e^{-r\left(\tau_{1}-t\right)} \widetilde{G}\left(W_{\tau_{1}-}, Y_{\tau_{1}-}\right) \mathcal{I}_{\tau_{1}=\tau_{F_{N}}^{1}}\right] \\
& \geq \mathbb{E}_{t}\left[e^{-r\left(\tau_{1}-t\right)} \sup _{F_{1} \geq 0}\left[\widetilde{G}\left(W_{\tau_{1}-}+F_{1}, Y_{\tau_{1}-}\right)-F_{1}-\Phi\left(F_{1}\right)\right] \mathcal{I}_{\tau_{1}=\tau_{F_{N}}^{1}<\tau_{i}}\right. \\
& \left.+e^{-r\left(\tau_{1}-t\right)} \sup _{F_{1} \geq 0}\left[P\left(W_{\tau_{1}-}+F_{1}-I, Y_{\tau_{1}-}\right)-F_{1}-\Phi\left(F_{1}\right)\right] \mathcal{I}_{\tau_{1}=\tau_{F_{N}}^{1}}=\tau_{i}\right] \\
& =\mathbb{E}_{t}\left[e^{-r\left(\tau_{1}-t\right)} \sup _{F_{1} \geq 0}\left[\mathbb{E}_{\tau_{F_{N}}^{1}}\left[\widetilde{G}\left(W_{\tau_{1}-}+F_{1}, Y_{\tau_{1}-}\right)\right]-F_{1}-\Phi\left(F_{1}\right)\right] \mathcal{I}_{\tau_{1}=\tau_{F_{N}}^{1}<\tau_{i}}\right. \\
& \left.+e^{-r\left(\tau_{1}-t\right)} \sup _{F_{1} \geq 0}\left[\mathbb{E}_{\tau_{F_{N}}^{1}}\left[P\left(W_{\tau_{1}-}+F_{1}-I, Y_{\tau_{1}-}\right)\right]-F_{1}-\Phi\left(F_{1}\right)\right] \mathcal{I}_{\tau_{1}=\tau_{F_{N}}^{1}}=\tau_{i}\right] \\
& \geq \mathbb{E}_{t}\left[e^{-r\left(\tau_{1}-t\right)}\left[\mathbb{E}_{\tau_{F_{N}}^{1}}\left[G\left(W_{\tau_{1}-}+F_{1}, Y_{\tau_{1}-}\right)\right]-F_{1}-\Phi\left(F_{1}\right)\right] \mathcal{I}_{\tau_{1}=\tau_{F_{N}}^{1}<\tau_{i}}\right. \\
& \left.+e^{-r\left(\tau_{1}-t\right)}\left[\mathbb{E}_{\tau_{F_{N}}^{1}}\left[P\left(W_{\tau_{1}-}+F_{1}-I, Y_{\tau_{1}-}\right)\right]-F_{1}-\Phi\left(F_{1}\right)\right] \mathcal{I}_{\tau_{1}=\tau_{F_{N}}^{1}}=\tau_{i}\right] \\
& =G\left(W_{t}, Y_{t} ; A\right) \text {, }
\end{aligned}
$$

where again we have used the condition (C.5) for the first term of the second inequality and the condition (C.6) for the second term of the second inequality, and the third inequality is obtained from (C.7).

Following similar steps as for earlier subperiods we obtain that $\widetilde{G}\left(W_{t}, Y_{t}\right) \geq$ $G\left(W_{t}, Y_{t} ; A\right)$. Since the policy $A$ is arbitrarily chosen, we have

$$
\widetilde{G}(W, Y) \geq \max _{A \in \mathcal{A}} G(W, Y ; A)=G(W, Y) .
$$


Finally, since the optimal strategies are attained, all the inequalities become equalities, so that:

$$
\widetilde{G}(W, Y)=G(W, Y ; A)=G(W, Y),
$$

when $A=A^{*}$, where

$$
A^{*} \in \mathcal{A}^{*}=\left\{\arg \max _{A \in \mathcal{A}} G(W, Y ; A)\right\} .
$$

\section{C.7 Proof for Proposition 3}

Proof for the Existence and Boundedness Property. It is immediate to observe that the closed-form solution for $G^{*}(W, Y)$ with $(W, Y) \in \Sigma_{1}$ is bounded under Assumptions 1-3. It follows that $G(W, Y)$ is also bounded under Assumptions 1-3.

Lemma 5 shows that $W \leq G(W, Y)$. To see why $G(W, Y) \leq G^{*}(W, Y)$, let $G_{(1)}(W, Y)$ be the value function if the firm is allowed to issue equity freely, without paying any external financing cost in the first round of financing. We then have:

$$
\begin{aligned}
G\left(W_{t}, Y_{t}\right)= & \mathbb{E}_{t}\left[e^{-r\left(\tau_{1}-t\right)}\left[G\left(W_{\tau_{1}-}+F_{1}, Y_{\tau_{1}-}\right)-F_{1}-\Phi\left(F_{1}\right)\right] \mathcal{I}_{\tau_{1}=\tau_{F}^{1}<\tau_{i}}\right. \\
& +e^{-r\left(\tau_{1}-t\right)}\left[P\left(W_{\tau_{1}-}+F_{1}-I, Y_{\tau_{1}-}\right)-F_{1}-\Phi\left(F_{1}\right)\right] \mathcal{I}_{\tau_{1}=\tau_{F}^{1}=\tau_{i}} \\
& \left.+e^{-r\left(\tau_{1}-t\right)} W_{\tau_{1}} \mathcal{I}_{\tau_{1}=\tau_{L}^{1}}+e^{-r\left(\tau_{1}-t\right)} G^{*}\left(W_{\tau_{1}}, Y_{\tau_{1}}\right) \mathcal{I}_{\tau_{1}=\tau_{U}^{1}}\right] \\
\leq & \mathbb{E}_{t}\left[e^{-r\left(\tau_{1}-t\right)}\left[G\left(W_{\tau_{1}-}+F_{1}, Y_{\tau_{1}-}\right)-F_{1}\right] \mathcal{I}_{\tau_{1}=\tau_{F}^{1}<\tau_{i}}+e^{-r\left(\tau_{1}-t\right)} W_{\tau_{1}} \mathcal{I}_{\tau_{1}=\tau_{L}^{1}}\right. \\
& +e^{-r\left(\tau_{1}-t\right)}\left[P\left(W_{\tau_{1}-}+F_{1}-I, Y_{\tau_{1}-}\right)-F_{1}\right] \mathcal{I}_{\tau_{1}=\tau_{F}^{1}=\tau_{i}} \\
& \left.+e^{-r\left(\tau_{1}-t\right)} G^{*}\left(W_{\tau_{1}}, Y_{\tau_{1}}\right) \mathcal{I}_{\tau_{1}=\tau_{U}^{1}}\right] \\
= & G_{(1)}\left(W_{t}, Y_{t}\right) .
\end{aligned}
$$

Now, let $G_{(2)}(W, Y)$ be the value function if the firm has the opportunity to issue equity without any cost for the next two rounds. Then:

$$
\begin{aligned}
G_{(1)}\left(W_{t}, Y_{t}\right)=\mathbb{E}_{t}\left[e^{-r\left(\tau_{1}-t\right)}\left[G\left(W_{\tau_{1}-}+F_{1}, Y_{\tau_{1}-}\right)-F_{1}\right] \mathcal{I}_{\tau_{1}=\tau_{F}^{1}<\tau_{i}}\right. \\
+e^{-r\left(\tau_{1}-t\right)}\left[P\left(W_{\tau_{1}-}+F_{1}-I, Y_{\tau_{1}-}\right)-F_{1}\right] \mathcal{I}_{\tau_{1}=\tau_{F}^{1}=\tau_{i}} \\
\left.+e^{-r\left(\tau_{1}-t\right)} W_{\tau_{1}} \mathcal{I}_{\tau_{1}=\tau_{L}^{1}}+e^{-r\left(\tau_{1}-t\right)} G^{*}\left(W_{\tau_{1}}, Y_{\tau_{1}}\right) \mathcal{I}_{\tau_{1}=\tau_{U}^{1}}\right] \\
\leq \mathbb{E}_{t}\left[e^{-r\left(\tau_{1}-t\right)}\left[G_{(1)}\left(W_{\tau_{1}-}+F_{1}, Y_{\tau_{1}-}\right)-F\right] \mathcal{I}_{\tau_{1}=\tau_{F}^{1}<\tau_{i}}\right. \\
\quad+e^{-r\left(\tau_{1}-t\right)}\left[P\left(W_{\tau_{1}-}+F_{1}-I, Y_{\tau_{1}-}\right)-F\right] \mathcal{I}_{\tau_{1}=\tau_{F}^{1}=\tau_{i}} \\
\left.\quad+e^{-r\left(\tau_{1}-t\right)} W_{\tau_{1}} \mathcal{I}_{\tau_{1}=\tau_{L}^{1}}+e^{-r\left(\tau_{1}-t\right)} G^{*}\left(W_{\tau_{1}}, Y_{\tau_{1}}\right) \mathcal{I}_{\tau_{1}=\tau_{U}^{1}}^{1}\right] \\
=G_{(2)}\left(W_{t}, Y_{t}\right) .
\end{aligned}
$$


Repeating the same argument, we have

$$
G(W, Y) \leq G_{(1)}(W, Y) \leq G_{(2)}(W, Y) \leq \cdots \leq G_{(\infty)}(W, Y)
$$

where $G_{(\infty)}(W, Y)$ represents the value function for an infinite number of costless issues. In other words, $G_{(\infty)}(W, Y)$ is the firm's value when it is forever financially unconstrained, so that $G_{(\infty)}(W, Y) \leq G^{*}(W, Y)$. It then follows that:

$$
G(W, Y) \leq G^{*}(W, Y)
$$

Finally, we establish that $G(W, Y)$ exists under Assumption 2 and Assumption 3. Note first that the set of policies $\mathcal{A}$ is non-empty. Indeed, one policy $A \in \mathcal{A}$ is to immediately liquidate the firm at time $t, A=\left\{\left(\tau_{i}>t, \tau_{F}^{1}>t, \tau_{L}^{1}=t, F_{1}=0\right)\right\}$. The firm's payoff under this policy is $G\left(W_{t}, Y_{t} ; A\right)=W_{t}$. Therefore, there exists at least one policy $A^{*} \in \mathcal{A}$ such that $G\left(W_{t}, Y_{t} ; A^{*}\right)=\max _{A \in \mathcal{A}} G\left(W_{t}, Y_{t} ; A\right)$. Note that $G\left(W_{t}, Y_{t}\right)=\max _{A \in \mathcal{A}} G\left(W_{t}, Y_{t} ; A\right)$, so that it exists and is given by $G\left(W_{t}, Y_{t}\right)=$ $G\left(W_{t}, Y_{t} ; A^{*}\right)$.

Proof for the Monotonicity Property. First we show that $G(W, Y)$ is strictly increasing in $W$. For simplicity, define $H\left(W_{t}, Y_{t} ; A\right)=G\left(W_{t}, Y_{t} ; A\right)-W$, and from (29) we have

$$
\begin{aligned}
& H\left(W_{t}, Y_{t} ; A\right)=\mathbb{E}_{t}\left[e^{-r\left(\tau_{1}-t\right)}\left[Q\left(W_{\tau_{1}-}+F_{1}-I, Y_{\tau_{1}-}\right)-\Phi\left(F_{1}\right)\right] \mathcal{I}_{\tau_{1}=\tau_{i}}\right. \\
& \left.\quad+e^{-r\left(\tau_{1}-t\right)} H^{*}\left(Y_{\tau_{1}}\right) \mathcal{I}_{\tau_{1}=\tau_{U}^{1}}+e^{-r\left(\tau_{1}-t\right)}\left[H\left(W_{\tau_{1}-}+F_{1}, Y_{\tau_{1}-}\right)-\Phi\left(F_{1}\right)\right] \mathcal{I}_{\tau_{1}=\tau_{F}^{1}}\right],
\end{aligned}
$$

and

$$
H\left(W_{t}, Y_{t}\right)=\sup _{A \in \mathcal{A}} H\left(W_{t}, Y_{t} ; A\right) .
$$

Obviously, $G(W, Y)$ is strictly increasing in $W$ if $H(W, Y)$ is increasing in $W$. Next, we prove that $H(W, Y)$ is increasing in $W$.

For the firm with initial wealth $W^{(1)}$, a policy $A_{1}$ can be constructed with the same investment, the same financing time, and the same liquidation time as policy $A_{2}^{*}$, where the financing amount is given by $F_{1}^{1}=F_{1}^{2, *}+W_{s-}^{(2)}-W_{s-}^{(1)}$ at the time of financing. Observe next that the dynamics of liquidity are then such that $W_{s}^{(1)} \geq$ $W_{s}^{(2)}$ for $s>t$, path by path, if $W_{t}^{(1)}>W_{t}^{(2)}$, which implies that $W_{\tau_{1}}^{(1)} \geq W_{\tau_{1}}^{(2)}=$ $\max \left\{\Lambda_{1}, \Lambda_{2}+I\right\}$ if $\tau_{1}=\tau_{U}^{2, *}$. In addition, we have $W_{s}^{(1)}=W_{s}^{(2)}$ and $F_{1}^{1}=F_{1}^{2, *}$ for $s>\tau_{1}=\min \left\{\tau_{i}^{2, *}, \tau_{F}^{1, *}\right\}$, and $F_{1}^{1} \leq F_{1}^{2, *}$ for $t<s \leq \tau_{1}=\min \left\{\tau_{i}^{2, *}, \tau_{F}^{2, *}\right\}$. 
Under this policy $A_{1}$ we then have:

$$
\begin{aligned}
H\left(W_{t}^{(1)}, Y_{t} ; A_{1}\right)= & \mathbb{E}_{t}\left[e^{-r\left(\tau_{1}-t\right)}\left[Q\left(W_{\tau_{1}-}^{(1)}+F_{1}^{1}-I, Y_{\tau_{1}-}\right)-\Phi\left(F_{1}^{1}\right)\right] \mathcal{I}_{\tau_{1}=\tau_{i}^{2, *}}\right. \\
& +e^{-r\left(\tau_{1}-t\right)} H^{*}\left(Y_{\tau_{1}}\right) \mathcal{I}_{\tau_{1}=\tau_{U}^{2, *}} \\
& \left.+e^{-r\left(\tau_{1}-t\right)}\left[H\left(W_{\tau_{1}-}^{(1)}+F_{1}^{1}, Y_{\tau_{1}-}\right)-\Phi\left(F_{1}^{1}\right)\right] \mathcal{I}_{\tau_{1}=\tau_{F}^{2, *}}\right] \\
\geq \quad & \mathbb{E}_{t}\left[e^{-r\left(\tau_{1}-t\right)}\left[Q\left(W_{\tau_{1}-}^{(2)}+F_{1}^{2, *}-I, Y_{\tau_{1}-}\right)-\Phi\left(F_{1}^{2, *}\right)\right] \mathcal{I}_{\tau_{1}=\tau_{i}^{2, *}}\right. \\
& +e^{-r\left(\tau_{1}-t\right)} H^{*}\left(Y_{\tau_{1}}\right) \mathcal{I}_{\tau_{1}=\tau_{U}^{2, *}} \\
& \left.+e^{-r\left(\tau_{1}-t\right)}\left[H\left(W_{\tau_{1}-}^{(2)}+F_{1}^{2, *}, Y_{\tau_{1}-}\right)-\Phi\left(F_{1}^{2, *}\right)\right] \mathcal{I}_{\tau_{1}=\tau_{F}^{2, *}}\right] \\
= & H\left(W_{t}^{(2)}, Y_{t} ; A_{2}^{*}\right)=H\left(W_{t}^{(2)}, Y_{t}\right) .
\end{aligned}
$$

Hence, we have

$$
H\left(W_{t}^{(1)}, Y_{t}\right)=H\left(W_{t}^{(1)}, Y_{t} ; A_{1}^{*}\right) \geq H\left(W_{t}^{(1)}, Y_{t} ; A_{1}\right) \geq H\left(W_{t}^{(2)}, Y_{t} ; A_{2}^{*}\right)=H\left(W_{t}^{(2)}, Y_{t}\right)
$$

That is, $H(W, Y)$ is increasing in $W$ and $G(W, Y)=H(W, Y)+W$ is strictly increasing in $W$.

Second, we show that $G(W, Y)$ is strictly increasing in $Y$ for $Y \geq \underline{Y}(W)$. Note that $G(W, Y)$ is strictly increasing in $Y$ for $Y \geq \bar{Y}(W)$ since the firm exercises its growth option immediately and $P(W, Y)$ is strictly increasing in $Y$ as shown in Proposition 1. Therefore, we need to show that $G\left(W, Y^{(1)}\right)>G\left(W, Y^{(2)}\right)$ if $\bar{Y}(W)>Y^{(1)}>$ $Y^{(2)} \geq \underline{Y}(W)$. We can use the same argument as for the monotonicity in $W$. Again, we define the policies $A_{1}, A_{1}^{*}$ and $A_{2}^{*}$ as above, and let $A_{1}=A_{2}^{*}$. Furthermore, we let $W^{(1)}$ and $W^{(2)}$ denote the liquidity of the firm with initial earnings fundamentals $Y^{(1)}$ and $Y^{(2)}$ respectively. Observe next that the dynamics of liquidity are then such that $W_{s}^{(1)}>W_{s}^{(2)}$ for $t<s$, path by path, if $W_{t}^{(1)}=W_{t}^{(2)}$. This further implies that $W_{s}^{(1)}>W_{s}^{(2)}$ for $s=\tau_{1}=\min \left\{\tau_{i}^{2, *}, \tau_{L}^{2, *}, \tau_{U}^{2, *}\right\}$.

Since $G^{*}(W, Y)$ is always strictly increasing in $W$ and $Y$, the terminal payoff with initial earnings fundamentals $Y^{(1)}$ is always higher than with initial earnings fundamentals $Y^{(2)}$, path by path, under the same policy $A_{2}^{*}$, so that:

$$
G\left(W, Y^{(1)} ; A_{1}\right)=G\left(W, Y^{(1)} ; A_{2}^{*}\right)>G\left(W, Y^{(2)} ; A_{2}^{*}\right)=G\left(W, Y^{(2)}\right)
$$

Furthermore, $G\left(W, Y^{(1)}\right)=G\left(W, Y^{(1)} ; A_{1}^{*}\right) \geq G\left(W, Y^{(1)} ; A_{1}\right)$, so that:

$$
G\left(W, Y^{(1)}\right)>G\left(W, Y^{(2)}\right) \quad \text { for } \quad \bar{Y}(W)>Y^{(1)}>Y^{(2)} \geq \underline{Y}(W) .
$$

Finally, since $G(W, Y)$ is strictly increasing in $Y$ for $Y \geq \bar{Y}(W)$, we have that $G(W, Y)$ is strictly increasing in $Y$ for $Y \geq \underline{Y}(W)$. 
Proof for the Uniqueness Property. Let $\Sigma_{G}$ denote the interior region where $\mathcal{L}_{1} G=0$ :

$$
\Sigma_{G}=\left\{(W, Y) \mid 0<W<\max \left\{\Lambda_{1}, \Lambda_{2}+I\right\}, \underline{Y}(W)<Y<\bar{Y}\right\}
$$

Let $\Sigma_{G}^{\prime}$ denote the boundary of $\Sigma_{G}$ :

$$
\begin{aligned}
\Sigma_{G}^{\prime}= & \left\{(W, Y) \mid W=\left\{0, \max \left\{\Lambda_{1}, \Lambda_{2}+I\right\}\right\}, \underline{Y}(W) \leq Y<\bar{Y}(W)\right\} \\
& \cup\left\{(W, Y) \mid 0 \leq W \leq \max \left\{\Lambda_{1}, \Lambda_{2}+I\right\}, Y=\{\underline{Y}(W), \bar{Y}(W)\}\right\} .
\end{aligned}
$$

We first show that the solution is unique for a given region $\Sigma_{G}$ and then show that $\Sigma_{G}$ is unique. Finally, we show that the solution is unique on the whole region $\widehat{\Sigma}_{1}=\Sigma_{G} \cup \Sigma_{G}^{\prime}$.

Suppose by contradiction that for a given region $\Sigma_{G}$ where $\mathcal{L}_{1} G=0$, there are two solutions $G^{(1)}(W, Y)$ and $G^{(2)}(W, Y)$ which satisfy:

$$
0=\left(r W+m_{1} Y-Z_{1}\right) G_{W}^{(1)}(W, Y)+\mu Y G_{Y}^{(1)}(W, Y)+\frac{\sigma^{2} Y^{2} G_{Y Y}^{(1)}(W, Y)}{2}-r G^{(1)}(W, Y)
$$

and

$0=\left(r W+m_{1} Y-Z_{1}\right) G_{W}^{(2)}(W, Y)+\mu Y G_{Y}^{(2)}(W, Y)+\frac{\sigma^{2} Y^{2} G_{Y Y}^{(2)}(W, Y)}{2}-r G^{(2)}(W, Y)$,

in the region $\Sigma_{G}$. Let $\widehat{G}(W, Y)=G^{(1)}(W, Y)-G^{(2)}(W, Y)$. Then $\widehat{G}(W, Y)$ also satisfies

$$
0=\left(r W+m_{1} Y-Z_{1}\right) \widehat{G}_{W}(W, Y)+\mu Y \widehat{G}_{Y}(W, Y)+\frac{\sigma^{2} Y^{2} \widehat{G}_{Y Y}(W, Y)}{2}-r \widehat{G}(W, Y),
$$

in region $\Sigma_{G}$. Let

$$
(\widehat{W}, \widehat{Y}) \in \arg \max _{(W, Y) \in \Sigma_{G} \cup \Sigma_{G}^{\prime}} \widehat{G}(W, Y)
$$

If $(\widehat{W}, \widehat{Y}) \in \Sigma_{G}$, we have that $\widehat{G}_{W}(\widehat{W}, \widehat{Y})=0, \widehat{G}_{Y}(\widehat{W}, \widehat{Y})=0$, and $\widehat{G}_{Y Y}(\widehat{W}, \widehat{Y}) \leq 0$. Under Assumption 2, we also have:

$$
r \widehat{G}(\widehat{W}, \widehat{Y})=\frac{\sigma^{2} \widehat{Y}^{2} \widehat{G}_{Y Y}(\widehat{W}, \widehat{Y})}{2} \leq 0
$$

Therefore,

$$
\widehat{G}(\widehat{W}, \widehat{Y}) \leq 0, \quad \text { if } \quad(\widehat{W}, \widehat{Y}) \in \Sigma_{G} .
$$

Note that $(W, \underline{Y}(W)) \in \Sigma_{G}^{\prime}$, so that

$\max _{(W, Y) \in \Sigma_{G} \cup \Sigma_{G}^{\prime}} \widehat{G}(W, Y) \geq \max _{(W, Y) \in \Sigma_{G}^{\prime}} \widehat{G}(W, Y) \geq G^{(1)}(W, \underline{Y}(W))-G^{(2)}(W, \underline{Y}(W))=W-W=0$. 
That is

$$
\widehat{G}(\widehat{W}, \widehat{Y}) \geq 0, \quad \text { for } \quad(\widehat{W}, \widehat{Y}) \in \Sigma_{G} \cup \Sigma_{G}^{\prime}
$$

Combining (C.13) and (C.15), we have

$$
\widehat{G}(\widehat{W}, \widehat{Y}) \equiv 0, \quad \text { if } \quad(\widehat{W}, \widehat{Y}) \in \Sigma_{G}
$$

Next, we prove that $\widehat{G}(\widehat{W}, \widehat{Y}) \equiv 0$ if $(\widehat{W}, \widehat{Y}) \in \Sigma_{G}^{\prime}$. By using the value functions at the boundaries: $Y=\{\underline{Y}(W), \bar{Y}(W)\}$ and $W=\max \left\{\Lambda_{1}, \Lambda_{2}+I\right\}$, we have

$$
\widehat{G}(\widehat{W}, \widehat{Y})=0
$$

if $(\widehat{W}, \widehat{Y}) \in\left\{(W, Y) \mid W=\max \left\{\Lambda_{1}, \Lambda_{2}+I\right\}, \underline{Y}(W) \leq Y<\bar{Y}(W)\right\} \cup\{(W, Y) \mid 0 \leq W \leq$ $\left.\max \left\{\Lambda_{1}, \Lambda_{2}+I\right\}, Y=\{\underline{Y}(W), \bar{Y}(W)\}\right\}$.

Next we show that $\widehat{G}(\widehat{W}, \widehat{Y})=0, \quad$ if $\quad(\widehat{W}, \widehat{Y}) \in\{(W, Y) \mid W=0, \underline{Y}(W) \leq Y<$ $\bar{Y}(W)\}$. First, note that $\widehat{G}_{W}(\widehat{W}, \widehat{Y}) \leq 0, \widehat{G}_{Y}(\widehat{W}, \widehat{Y})=0$ and $\widehat{G}_{Y Y}(\widehat{W}, \widehat{Y}) \leq 0$ if $\widehat{W}=0$, and $\frac{Z_{1}}{m_{1}} \leq \widehat{Y}<\bar{Y}(W)$. Therefore, we have $r \widehat{P}(0, \widehat{Y})=\left(m_{1} \widehat{Y}-Z_{1}\right) \widehat{G}_{W}(W, Y)+$ $\frac{\sigma^{2} \widehat{Y}^{2} \widehat{G}_{Y Y}(0, \widehat{Y})}{2} \leq 0$ if $\frac{Z_{1}}{m_{1}} \leq \widehat{Y}<\bar{Y}(W)$. Then from (C.15), we obtain that

$$
\widehat{G}(0, \widehat{Y})=0, \quad \text { if } \quad \frac{Z_{1}}{m_{1}} \leq \widehat{Y}<\bar{Y}(W) .
$$

Finally, let $F_{1}^{(1)}$ and $F_{1}^{(2)}$ denote the optimal equity issues in that $G^{(1)}(0, \widehat{Y})=$ $\sup _{F_{1}>0} G^{(1)}\left(F_{1}, \widehat{Y}\right)-F_{1}-\Phi\left(F_{1}\right)$ and $G^{(2)}(0, \widehat{Y})=\sup _{F_{1}>0} G^{(2)}\left(F_{1}, \widehat{Y}\right)-F_{1}-\Phi\left(F_{1}\right)$. We then have

$$
\begin{aligned}
\widehat{G}(0, \widehat{Y}) & =G^{(1)}(0, \widehat{Y})-G^{(2)}(0, \widehat{Y}) \\
& =\left(G^{(1)}\left(F_{1}^{(1)}, \widehat{Y}\right)-F_{1}^{(1)}-\Phi\left(F_{1}^{(1)}\right)\right)-\left(G^{(2)}\left(F_{1}^{(2)}, \widehat{Y}\right)-F_{1}^{(2)}-\Phi\left(F_{1}^{(2)}\right)\right) \\
& \leq\left(G^{(1)}\left(F_{1}^{(1)}, \widehat{Y}\right)-F_{1}^{(1)}-\Phi\left(F_{1}^{(1)}\right)\right)-\left(G^{(2)}\left(F_{1}^{(1)}, \widehat{Y}\right)-F_{1}^{(1)}-\Phi\left(F_{1}^{(1)}\right)\right) \\
& =\widehat{G}\left(F_{1}^{(1)}, \widehat{Y}\right) .
\end{aligned}
$$

This is obviously a contradiction unless $\widehat{G}(W, Y) \equiv 0$. That is $\widehat{G}(0, \widehat{Y})=0$ if $\underline{Y}(0) \leq \widehat{Y}<\frac{Z_{1}}{m_{1}}$. Together with (C.18) and (C.17), we therefore conclude that

$$
\widehat{G}(\widehat{W}, \widehat{Y}) \equiv 0, \quad \text { if } \quad(\widehat{W}, \widehat{Y}) \in \Sigma_{G}^{\prime}
$$

Further, by combining (C.16) and (C.20), we conclude that

$$
\widehat{G}(\widehat{W}, \widehat{Y}) \equiv 0 \quad \text { for } \quad(W, Y) \in \Sigma_{G} \cup \Sigma_{G}^{\prime}
$$

This, in turn, implies that

$$
G^{(1)}(W, Y) \leq G^{(2)}(W, Y) \quad \text { for } \quad(W, Y) \in \Sigma_{G} \cup \Sigma_{G}^{\prime} .
$$


Since the entire argument also holds if we switch $G^{(1)}(W, Y)$ with $G^{(2)}(W, Y)$, in that

$$
G^{(2)}(W, Y) \leq G^{(1)}(W, Y) \quad \text { for } \quad(W, Y) \in \Sigma_{G} \cup \Sigma_{G}^{\prime},
$$

we conclude that

$$
G^{(2)}(W, Y) \equiv G^{(1)}(W, Y) \quad \text { for } \quad(W, Y) \in \Sigma_{G} \cup \Sigma_{G}^{\prime} .
$$

We next show that the region $\Sigma_{G}$ is unique, which is equivalent to showing that the boundary $\Sigma_{G}^{\prime}$ is unique. From the definition of $\Sigma_{G}^{\prime}$, we only need to show that $\underline{Y}(W)$ is unique and $\bar{Y}(W)$ is unique, for $0 \leq W \leq \Lambda_{2}$. First, suppose that there are two liquidation boundaries $\underline{Y}^{(1)}(W)$ and $\underline{Y}^{(2)}(W)$, with $\underline{Y}^{(1)}(W) \geq \underline{Y}^{(2)}(W)$ for some $W$, and let $G^{(1)}(W, Y)$ and $G^{(2)}(W, Y)$ be the corresponding solutions. We then have:

$$
W=G^{(1)}\left(W, \underline{Y}^{(1)}(W)\right) \geq G^{(2)}\left(W, \underline{Y}^{(1)}(W)\right) \geq W=G^{(2)}\left(W, \underline{Y}^{(2)}(W)\right),
$$

where the first inequality follows from the optimality of the liquidation decision, and the second inequality is obtained from (31). It follows that $G^{(2)}\left(W, \underline{Y}^{(1)}(W)\right)=$ $G^{(2)}\left(W, \underline{Y}^{(2)}(W)\right)$. Moreover, the value function is increasing in both $W$ and $Y$ so that: $\underline{Y}^{(1)}(W)=\underline{Y}^{(2)}(W)$.

Suppose next that there are two investment boundaries $\bar{Y}^{(1)}(W)$ and $\bar{Y}^{(2)}(W)$, with $\bar{Y}^{(1)}(W)>\bar{Y}^{(2)}(W)$ for some $W$, and let $G^{(1)}(W, Y)$ and $G^{(2)}(W, Y)$ be the corresponding solutions. It follows that $G^{(1)}\left(W, \bar{Y}^{(2)}(W)\right)>\sup _{F_{1}} P\left(W+F_{1}-\right.$ $\left.I, \bar{Y}^{(2)}(W)\right)$ since $\bar{Y}^{(2)}(W)$ is not the optimal investment boundary for $G^{(1)}(W, Y)$. We then have:

$$
G^{(1)}\left(W, \bar{Y}^{(1)}(W)\right)>G^{(1)}\left(W, \bar{Y}^{(2)}(W)\right)>\sup _{F_{1}} P\left(W+F_{1}-I, \bar{Y}^{(2)}(W)\right)=G^{(2)}\left(W, \bar{Y}^{(2)}(W)\right),
$$

where the first inequality follows from the monotonicity property shown in Proposition 3. Since $\bar{Y}^{(2)}(W)$ is the optimal investment boundary for $G^{(2)}(W, Y)$, we have $G^{(2)}\left(W, \bar{Y}^{(2)}(W)\right)>G^{(1)}\left(W, \bar{Y}^{(2)}(W)\right)$, which is conflicting with (C.24). Hence, we have that $\bar{Y}^{(1)}(W)=\bar{Y}^{(2)}(W)$ for all $W$.

Finally, we show that the solution is unique on the remaining liquidation/investment region $\Sigma_{1}^{\prime}=\Sigma_{1}-\left(\Sigma_{G} \cup \Sigma_{G}^{\prime}\right)$. From the definition of the regions $\Sigma_{G}$ and $\Sigma_{G}^{\prime}$, and total region $\Sigma_{1}$, we have that

$$
\begin{aligned}
\Sigma_{1}^{\prime}= & \left\{(W, Y) \mid 0 \leq W \leq \max \left\{\Lambda_{1}, \Lambda_{2}+I\right\}, 0 \leq Y<\underline{Y}(W)\right\} \\
& \cup\left\{(W, Y) \mid 0 \leq W \leq \max \left\{\Lambda_{1}, \Lambda_{2}+I\right\}, \bar{Y}(W)<Y\right\} .
\end{aligned}
$$

Since both of $\underline{Y}(W)$ and $\bar{Y}(W)$ are unique it follows that $\Sigma_{1}^{\prime}$ is also unique. In the liquidation region the value function is $G(W, Y) \equiv W$, and in the investment region 
the the value function is $G(W, Y) \equiv \sup _{F_{1} \geq 0} P\left(W+F_{1}-I, Y\right)$, so that the solution for $P\left(W+F_{1}-I, Y\right)$ is unique as shown in Proposition 1. Hence, the solution for $G(W, Y)$ is unique in $\Sigma_{1}^{\prime}$. In sum, the solution for $G(W, Y)$ in the region $\Sigma_{1}$ is unique.

\section{C.8 Proof for Proposition 4}

Proof for the Monotonicity Property of the Liquidation Boundary. Here, we want to show the optimal liquidation boundary, $\underline{Y}(W)$, is decreasing in $W$ in the growth phase. Let $\underline{Y}\left(W^{(1)}\right)$ and $\underline{Y}\left(W^{(2)}\right)$ denote the optimal liquidation boundary with respectively liquidity $W^{(1)}$ and $W^{(2)}$, where $W^{(1)}<W^{(2)}$. Recall that $H(W, Y)=G(W, Y)-W$ is increasing in $W$ as shown in Proposition 3. We therefore have $0=H\left(W^{(1)}, \underline{Y}\left(W^{(1)}\right)\right) \leq H\left(W^{(2)}, \underline{Y}\left(W^{(1)}\right)\right)$. In addition, $G(W, Y)$ is strictly increasing in $Y$ before liquidation as shown in Proposition 3. This implies that $H(W, Y)=G(W, Y)-W$ is also strictly increasing in $Y$ for $Y \geq \underline{Y}(W)$. Hence, we have $\underline{Y}\left(W^{(1)}\right) \geq \underline{Y}\left(W^{(2)}\right)$, otherwise $0=H\left(W^{(1)}, \underline{Y}\left(W^{(1)}\right)\right) \leq H\left(W^{(2)}, \underline{Y}\left(W^{(1)}\right)\right)<$ $H\left(W^{(2)}, \underline{Y}\left(W^{(2)}\right)\right)=0$, a contradiction. That is, we have established that $\underline{Y}\left(W^{(1)}\right) \geq$ $\underline{Y}\left(W^{(2)}\right)$ if $W^{(1)}<W^{(2)}$, which shows that the optimal liquidation boundary is decreasing in $W$ in the growth phase.

Proof for Delaying Costly Equity Issuance. First, we need to show that the firm has to issue equity if $W=0$ for given $Y$, where $\underline{Y}(0)<Y<\frac{Z_{1}}{m_{1}}$, to continue operations. We assume that $F_{1}^{*}(0)$ is the optimal amount of equity issuance, so that $G(0, Y)=G\left(F_{1}^{*}(0), Y\right)-F_{1}^{*}(0)-\Phi\left(F_{1}^{*}(0)\right)$. This implies that $G_{W}(0, Y)>$ $\left.1+\Phi^{* \prime}(0)\right)=1+\phi_{1}$, for otherwise $F_{1}^{*}(0)=0$ and the firm defaults immediately, which is a contradiction with $\underline{Y}(0)<Y<\frac{Z_{1}}{m_{1}}$. Next, denoting $M=F_{1}^{*}(0)$, we have that $G_{W}(W, Y)>1+\phi_{1}$ for $0<W<M$ and $G_{W}(M, Y)=1+\phi_{1}$. Otherwise, $F_{1}^{*}(0)$ is not the optimal amount of equity issuance.

To show that the firm will delay costly equity issuance until entirely exhausting its cash, we only need to show that $G(W, Y)>\sup _{F_{1} \geq 0} G\left(W+F_{1}, Y\right)-F_{1}-\Phi\left(F_{1}\right)$ for $W>0$. First, since $G_{W}(W, Y) \leq 1+\phi_{1}$ for $W \geq M$, the firm has no incentive to issue equity because $G(W, Y) \geq G\left(W+F_{1}, Y\right)-F_{1}-\phi_{1} F_{1}>G\left(W+F_{1}, Y\right)-F_{1}-\Phi\left(F_{1}\right)$ for $W \geq M$. Second, we show that $G(W, Y)>\sup _{F_{1} \geq 0} G\left(W+F_{1}, Y\right)-F_{1}-\Phi\left(F_{1}\right)$ for $0<W<M$. Let $F_{1}^{*}(W)$ be the optimal amount of equity issuance if the firm chooses equity issuance at some $W$ such that $0<W<M$ for given $Y$. This means that $\sup _{F_{1} \geq 0} G\left(W+F_{1}, Y\right)-F_{1}-\Phi\left(F_{1}\right)=G\left(W+F_{1}^{*}(W), Y\right)-F_{1}^{*}(W)-\Phi\left(F_{1}^{*}(W)\right)$ 
and $M=W+F_{1}^{*}(W)$. We then have

$$
\begin{aligned}
G & (W, Y)-\left(G\left(W+F_{1}^{*}(W), Y\right)-F_{1}^{*}(W)-\Phi\left(F_{1}^{*}(W)\right)\right) \\
& =G(W, Y)-\left(G(M, Y)-F_{1}^{*}(W)-\Phi\left(F_{1}^{*}(W)\right)\right) \\
& =G(W, Y)-W-\left(G(M, Y)-M-\Phi\left(F_{1}^{*}(W)\right)\right) \\
& =H(W, Y)-H(M, Y)+\Phi(M-W) .
\end{aligned}
$$

Recall that $G(0, Y)=G\left(F_{1}^{*}(0), Y\right)-F_{1}^{*}(0)-\Phi\left(F_{1}^{*}(0)\right)$ and $M=F_{1}^{*}(0)$, so that $H(0, Y)=H\left(F_{1}^{*}(0), Y\right)-\Phi\left(F_{1}^{*}(0)\right)=H(M, Y)-\Phi(M)$, and

$$
\begin{aligned}
& G(W, Y)-\left(G\left(W+F_{1}^{*}(W), Y\right)-F_{1}^{*}(W)-\Phi\left(F_{1}^{*}(W)\right)\right) \\
& \quad=H(W, Y)-H(0, Y)+\Phi(M-W)-\Phi(M) \\
& \quad=H(W, Y)-H(0, Y)-\phi_{1} W \\
& \quad>\phi_{1} W-\phi_{1} W=0
\end{aligned}
$$

where the above inequality follows from $G_{W}(W, Y)>1+\phi_{1}$ for $0<W<M$, which implies that $H_{W}(W, Y)>\phi_{1}$ for $0<W<M$. Finally, $G(W, Y)-(G(W+$ $\left.\left.F^{*}(W), Y\right)-F^{*}(W)-\Phi\left(F^{*}(W)\right)\right)>0$ implies that the firm has no incentive to issue equity for $0<W<M$ and that the firm will delay costly equity issuance until entirely exhausting its cash.

Proof for the behavior of the investment threshold when $W \rightarrow I$. If $P_{W}(0, \bar{Y}(I)) \leq \phi_{1}$, the firm delays costly equity issuance as shown in Proposition 2 , so that $P(0, \bar{Y}(I))>P\left(F_{1}, \bar{Y}(I)\right)-F_{1}-\phi_{1} F_{1}>P\left(F_{1}, \bar{Y}(I)\right)-F_{1}-\Phi\left(F_{1}\right)$ for any $0<F_{1} \leq M$ where $P_{W}(M, \bar{Y}(I))=\phi_{1}$. Hence, we have $G(I, \bar{Y}(I))=$ $\sup _{F_{1} \geq 0} P\left(F_{1}, \bar{Y}(I)\right)-F_{1}-\Phi\left(F_{1}\right)=P(0, \bar{Y}(I))$ if $P_{W}(0, \bar{Y}(I)) \leq \phi_{1}$. That is, the firm directly uses its internal liquidity to finance the investment cost $I$ if the marginal value of internal liquidity is lower than the external financing cost.

Denote $\bar{Y}(I-)=\lim _{W \rightarrow I} \bar{Y}(W)$. Now we show that $\bar{Y}(I-)=\infty$. First, we have $\bar{Y}(I-) \geq \bar{Y}(I)$, otherwise if $\bar{Y}(I-)<\bar{Y}(I)$ for given $\bar{Y}(I-)$ we have

$$
\begin{aligned}
\lim _{W \rightarrow I} G(W, \bar{Y}(I-)) & =\lim _{W \rightarrow I} \sup _{F_{1}>0} P\left(W+F_{1}-I, \bar{Y}(I-)\right)-F_{1}-\Phi\left(F_{1}\right) \\
& =\sup _{F_{1}>0} P\left(I+F_{1}-I, \bar{Y}(I-)\right)-F_{1}-\Phi\left(F_{1}\right)<G(I, \bar{Y}(I-)),
\end{aligned}
$$

where the second equality follows from the continuity of $G(W, Y)$, and the inequality from $\bar{Y}(I-)<\bar{Y}(I)$ and the fact that $\sup _{F} P\left(W+F_{1}-I, \bar{Y}(I-)\right)-F_{1}-\Phi\left(F_{1}\right)<$ $G(W, Y)$ if $Y<\bar{Y}(W)$. Hence, we have that $\bar{Y}(I-) \geq \bar{Y}(I)$. 
Now, assume that $\bar{Y}(I) \leq \bar{Y}(I-)<\infty$, then we have

$$
\begin{aligned}
\lim _{W \rightarrow I} G(W, \bar{Y}(I-)) & =\lim _{W \rightarrow I} \sup _{F_{1}>0} P\left(W+F_{1}-I, \bar{Y}(I-)\right)-F_{1}-\Phi\left(F_{1}\right) \\
& =\sup _{F_{1}>0} P\left(I+F_{1}-I, \bar{Y}(I-)\right)-F_{1}-\Phi\left(F_{1}\right) \\
& =\lim _{F_{1} \rightarrow 0} P\left(F_{1}, \bar{Y}(I-)\right)-F_{1}-\Phi\left(F_{1}\right) \\
& =P(0, \bar{Y}(I-))-\phi_{0}=G(I, \bar{Y}(I-))-\phi_{0} .
\end{aligned}
$$

The above result violates the continuity of $G(W, Y)$ in $W$ if $\bar{Y}(I) \leq \bar{Y}(I-)<\infty$. In sum, we have $\bar{Y}(I-) \rightarrow \infty$ if $P_{W}(0, \bar{Y}(I)) \leq \phi_{1}$, so that the investment threshold tends to infinity when $W \rightarrow I$ if $P_{W}(0, \bar{Y}(I)) \leq \phi_{1}$.

\section{Solution Algorithm}

First, we note that solving the following problem

$$
\max \left\{\mathcal{L}_{2} P, \max \left\{\sup _{F_{2} \geq 0} P\left(W+F_{2}, Y\right)-F_{2}-\Phi\left(F_{2}\right), W\right\}-P(W, Y)\right\}=0,
$$

in the region $(W \geq 0, Y \geq 0)$ is equivalent to solving the following penalty equation:

$$
\mathcal{L}_{2} P+q \max \{W-P, 0\}=0,
$$

in the region $\left[0, \Lambda_{2}\right] \times\left[0, Y_{\max }\right]$ where the approximate boundary $Y_{\max }$ and the penalty factor $q$ are sufficiently high. The following boundary conditions are associated with (D.2):

1. When the firm has no savings, i.e., $W=0$ :

$$
\begin{gathered}
P(0, Y)=\max \left\{\sup P\left(F_{2}, Y\right)-\phi_{0}-\left(1+\phi_{1}\right) F_{2}, 0\right\}, \quad \text { if } \quad Y<\frac{Z_{2}}{m_{2}} \\
\mathcal{L}_{2} P+q \max \{W-P, 0\}=0 \quad \text { if } \quad Y \geq \frac{Z_{2}}{m_{2}}
\end{gathered}
$$

2. When the firm's liquidity is sufficiently high, i.e., $W=\Lambda_{2}$ :

$$
\begin{aligned}
& P(W, Y)=W, \quad \text { if } Y<Y_{2, a}^{*} \\
& P(W, Y)=Q^{*}(Y)+W, \quad \text { if } \quad Y \geq Y_{2, a}^{*}
\end{aligned}
$$

3. When the firm's earnings is at the origin, $Y=0$, a permanently absorbing state, it is immediate to see that $P=W$. 
4. When the firm's earnings is sufficiently high, i.e., $Y=Y_{\max }$, the firm's can finance its operations in an unconstrained way with probability one. Therefore, $P\left(W, Y_{\max }\right)=P^{*}\left(W, Y_{\max }\right)=Q^{*}\left(Y_{\max }\right)+W$.

We use the finite-difference method to solve the penalty equation (D.2). That is, we divide the interval $\left[0, \Lambda_{2}\right]$ via $N_{w}$ equally spaced points $\left\{(i-1) \Delta_{w}\right\}_{i=1}^{N_{w}}$, where $\Delta_{w}=\Lambda_{2} /\left(N_{w}-1\right)$, and divide the interval $\left[0, Y_{\max }\right]$ via $N_{y}$ equally spaced points $\left\{(j-1) \Delta_{y}\right\}_{j=1}^{N_{y}}$, where $\Delta_{y}=Y_{\max } /\left(N_{y}-1\right)$. We then solve the penalty equation $\mathcal{L}_{2} P_{i, j}+q \max \left\{W_{i}-P_{i, j}, 0\right\}=0$ by using Newton-SOR iterative method, by setting $W_{i}=(i-1) \Delta_{w}, Y_{j}=(j-1) \Delta_{y}$, and $P_{i, j}=P\left(W_{i}, Y_{j}\right)$. Under Assumption 2 and Assumption (3), the value function is well defined and the numerical procedure converges. 


\section{References}

Abel, A.B., and J.C. Eberly, 1994. A unified model of investment under uncertainty. American Economic Review 84, 1369-1384.

Aiyagari, S. R., 1994. Uninsured idiosyncratic risk and aggregate saving. Quarterly Journal of Economics 99, 659-684.

Alfaro, I., N. Bloom, and X. Lin. 2016. The real and financial impact of uncertainty shocks. Stanford University Working Paper.

Anderson, R., and A. Carverhill, 2012. Corporate liquidity and capital structure. Review of Financial Studies 20, 1429-1460.

Asquith, P., and D. W. Mullins, 1986. Equity issues and offering dilution. Journal of Financial Economics 15(1-2), 61-89.

Babenko I., and Y. Tserlukevich, 2013. Embracing risk: Hedging policy for firms with real options, working paper, Arizona State University.

Baker, S. R., N. Bloom, and S. J. Davis, 2016. Measuring economic policy uncertainty. The Quarterly Journal of Economics 131(4), 1593-1636.

Baker, M., and J. Wurgler, 2002. Market timing and capital structure. The Journal of Finance 57, 1-32.

Black, F., and M. Scholes, 1973. The pricing of options and corporate liabilities. Journal of Political Economy 81, 637-659.

Bloom, N., 2009. The impact of uncertainty shocks. Econometrica 77, 623-685.

Bloom, N., Floetotto, M., Jaimovich, N., Saporta-Eksten, I., and S.J. Terry, 2016. Really uncertain business cycles. Stanford University Working Paper

Bolton, P., H. Chen, and N. Wang, 2011. A unified theory of Tobin' $q$, corporate investment, financing, and risk management. Journal of Finance 66, 1545-1578.

Bolton, P., H. Chen, and N. Wang, 2013. Market timing, investment, and risk management. Journal of Financial Economics 109, 40-62.

Bolton, P., N. Wang, and J. Yang, 2014. Investment under uncertainty and the value of real and financial flexibility (No. w20610). National Bureau of Economic Research.

Bolton, P., N. Wang, and J. Yang, 2019. Optimal Contracting, Corporate Finance, and Valuation with Inalienable Human Capital. Journal of Finance, 74, 13631429 . 
Boot, A.W.A., and V. N. Vladimirov, 2014. Entrepreneurial vision, information, and cash. Working paper. University of Amsterdam.

Boyle, G., and G. Guthrie, 2003. Investment, uncertainty, and liquidity. Journal of Finance 58, 2143-2166.

Brennan, M.J., and E. Schwartz, 1985. Evaluating natural resource investments. Journal of Business 58, 135-157.

Campello, M., J. R. Graham, and C. R. Harvey, 2010. The real effects of financial constraints: Evidence from a financial crisis. Journal of financial Economics $97(3), 470-487$.

Campello, M., Giambona, E., Graham, J. R., and Harvey, C. R. 2011. Liquidity management and corporate investment during a financial crisis. Review of Financial Studies 24(6), 1944-1979.

Carlson, M., A. Fisher, and R. Giammarino, 2006. Corporate investment and asset price dynamics: Implications for SEO event studies and long-run performance. The Journal of Finance 61, 1009-1034.

Carlson, M., A. Fisher, and R. Giammarino, 2010. SEO risk dynamics. Review of Financial Studies 23, 4026-4077.

Calomiris, C. W. and C. P. Himmelberg, 1997. Investment Banking Costs as a Measure of the Cost of Access to External Finance. Columbia University Working Paper.

Chen, H., J. Miao, and N. Wang, 2010. Entrepreneurial finance and non-diversifiable risk. Review of Financial Studies, 23, 4348-88.

Chen, P., L. Karabarbounis, and B. Neiman, 2017. The Global Rise of Corporate Saving. National Bureau of Economic Research.

Chen, G., 2017. Corporate savings, financing, and investment with aggregate uncertainty shocks. Columbia University Working Paper.

Copeland, T., and A. Lyasoff, 2013. Seeking $q$ : the marginal efficiency of liquidity and its effect on investment financing and valuation. Working paper. University of San Diego and Boston University.

Dayanik, S. and I. Karatzas, 2003. On the optimal stopping problem for onedimensional diffusions. Stochastic processes and their applications, 107(2), 173212.

DeAngelo, H., L. DeAngelo, and R.M. Stulz, 2010. Seasoned equity offerings, market timing, and the corporate lifecycle. Journal of Financial Economics 95, 275-295. 
Décamps, J.P., S. Gryglewicz, E. Morellec, and S. Villeneuve, 2017. Corporate policies with permanent and transitory shocks. Review of Financial Studies 30, $162-210$.

Décamps, J.P., T. Mariotti, J.C. Rochet, and S. Villeneuve, 2011. Free cash flow, issuance costs and stock prices. Journal of Finance 66, 1501-1544.

Décamps, J.P., and S. Villeneuve, 2007. Optimal dividend policy and growth option. Finance and Stochastics 11, 3-27.

Deuflhard, P., 2011. Newton methods for nonlinear problems: affine invariance and adaptive algorithms (Vol. 35). Springer.

Dixit, A.K., 1989. Entry and exit decisions under uncertainty. Journal of Political Economy 97, 620-638.

Dixit, A.K., and R.S. Pindyck, 1994. Investment under uncertainty. Princeton University Press, Princeton, NJ.

Duffie, D., 2010. Dynamic asset pricing theory. Princeton University Press.

Eckbo, B. Espen, R. W. Masulis, and O. Norli., 2007. Security offerings. Handbook of corporate finance: Empirical corporate finance, 1, 233-373.

Fama, E. F., and K. R. French, 2001. Disappearing dividends: changing firm characteristics or lower propensity to pay? Journal of Financial Economics 60, 3-43.

Froot, K.A., D.S. Scharfstein, and J.C. Stein, 1993. Risk management: Coordinating corporate investment and financing policies. The Journal of Finance 48, 16291658.

Gorbenko, A.S. and A. Malenko, 2017. The timing and method of payment in mergers when acquirers are financially constrained. The Review of Financial Studies, 31, 3937-3978.

Graham, J.R., and C. Harvey, 2001. The theory and practice of corporate finance: Evidence from the field. Journal of Financial Economics 60, 187-243.

Graham, J.R., and C. Harvey, 2002. How do CFOs make capital budgeting and capital structure decisions? Journal of Applied Corporate Finance 15, 8-23.

Grenadier, S.R., 1996. The strategic exercise of options: development cascades and overbuilding in real estate markets. Journal of Finance 51, 1653-1679.

Grenadier, S.R. and A. Malenko, 2010. A Bayesian approach to real options: The case of distinguishing between temporary and permanent shocks. The Journal of Finance, 65, 1949-1986. 
Grenadier, S.R. and A. Malenko, 2011. Real options signaling games with applications to corporate finance. The Review of Financial Studies 24, 3993-4036.

Grenadier, S.R., A. Malenko, and N. Malenko, 2016. Timing decisions in organizations: Communication and authority in a dynamic environment. American Economic Review. 106, 2552-81.

Grenadier, S.R., and N. Wang, 2005. Investment timing, agency, and information. Journal of Financial Economics 75, 493-533.

Grenadier, S.R., and N. Wang, 2007. Investment under uncertainty and timeinconsistent preferences. Journal of Financial Economics 84, 2-39.

Hackbarth, D., and D. Mauer, 2012. Optimal priority structure, capital structure, and investment. Review of Financial Studies 25, 747-96.

Hugonnier, J., S. Malamud, and E. Morellec, 2015. Capital supply uncertainty, cash holdings, and investment. Review of Financial Studies, 28(2), 391-445.

Ivashina, V., and D. Scharfstein. 2010. Bank lending during the financial crisis of 2008. Journal of Financial Economics 97, 319-338.

Kaplan, S.N., and L. Zingales, 1997. Do investment-cash flow sensitivities provide useful measures of financing constraints? Quarterly Journal of Economics 112, 169-215.

Karatzas, I., and S. Shreve, 1988. Brownian motion and stochastic calculus. Springer Science \& Business Media.

Kim, W., and M. S. Weisbach, 2008. Motivations for public equity offers: An international perspective. Journal of Financial Economics 87, 281-307.

Kyle, A.S., H. Ou-Yang, and W. Xiong. 2006. Prospect theory and liquidation decisions. Journal of Economic Theory 129 (1), 273-288

Lambrecht, B.M., and W.R. Perraudin, 2003. Real options and preemption under incomplete information. Journal of Economic Dynamics and Control 27, 619643.

Lambrecht, B.M., and S. C. Myers, 2007. A theory of takeovers and disinvestment. Journal of Finance 62, 809-845.

Lambrecht, B.M., and S. C. Myers, 2008. Debt and managerial rents in a real-options model of the firm. Journal of Financial Economics 89, 209-231.

Leland, H. E., 1994. Corporate debt value, bond covenants, and optimal capital structure. Journal of Finance 49, 1213-1252. 
Loughran, T., and Ritter, J. R. 1995. The new issues puzzle. The Journal of Finance $50(1), 23-51$.

Loughran, T., and Ritter, J. R. 1997. The operating performance of firms conducting seasoned equity offerings. The Journal of Finance 52(5), 1823-1850.

Lucas, D. J., and R. L. McDonald, 1990. Equity issues and stock price dynamics. The Journal of Finance 45, 1019-1043.

Mauer, D., and A.J. Triantis, 1994. Interactions of corporate financing and investment decisions: A dynamic framework. Journal of Finance 49, 1253-1277.

McDonald, R., and D. Siegel, 1985. Investment and the valuation of firms when there is an option to shut down. International economic review. 331-349.

McDonald, R., and D. Siegel, 1986. The value of waiting to invest. Quarterly Journal of Economics 101, 707-727.

Merton, R.C., 1973. Theory of rational option pricing. Bell Journal of Economics and Management Science 4, 141-183.

Miao, J., and N. Wang, 2007. Investment, consumption, and hedging under incomplete markets. Journal of Financial Economics 86, 608-642.

Miao, J., and N. Wang, 2011. Risk, uncertainty, and option exercise. Journal of Economic Dynamics and Control, 35, 442-461.

Miller, M. H., and D. Orr, 1966. A model of demand for money by firms. Quarterly Journal of Economics 80, 413-435.

Morellec, E., 2004. Can managerial discretion explain observed leverage ratios? Review of Financial Studies 17, 257-294.

Myers, S. C. 1977. Determinants of corporate borrowing. Journal of Financial Economics 5(2), 147-175.

Myers, S. C., and N. S. Majluf, 1984. Corporate financing and investment decisions when firms have information that investors do not have. Journal of Financial Economics 13, 187-221.

Orlov, D., A. Skrzypacz, A. and P. Zryumov, 2019. Persuading the Regulator To Wait. Journal of Political Economy. forthcoming.

Sundaresan, S., N. Wang, and J. Yang, 2015. Dynamic Investment, Capital Structure, and Debt Overhang. Review of Corporate Finance Studies 4, 1-42.

Titman, S., 1985. Urban land prices under uncertainty. American Economic Review $75,505-514$. 
Titman, S., and S. Tsyplakov, 2007. A dynamic model of optimal capital structure. Review of Finance 11(3), 401-451.

Wang C., N. Wang, and J. Yang, A unified model of entrepreneurship dynamics. 2012. Journal of Financial Economics, 106(1), 1-23.

Williams, J.T., 1991. Real estate development as an option. Journal of Real Estate Finance and Economics 4, 191-208. 